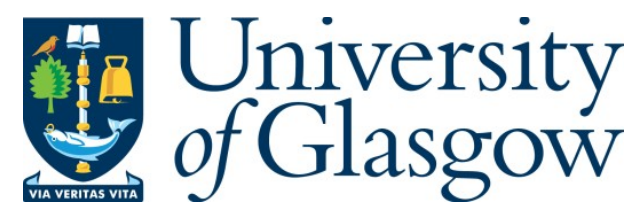

Obanda, A., Valerius, K., Mague, J. T., Sproules, S. and Donahue, J. P. (2020) Group 10 metal dithiolene bis(isonitrile) complexes: synthesis, structures, properties and reactivity. Organometallics, 39(15), pp. 2854-2870.

(doi: 10.1021/acs.organomet.0c00375)

This is the Author Accepted Manuscript.

There may be differences between this version and the published version. You are advised to consult the publisher's version if you wish to cite from it.

$\underline{\text { https://eprints.gla.ac.uk/219946/ }}$

Deposited on: 7 July 2020

Enlighten - Research publications by members of the University of Glasgow http://eprints.gla.ac.uk 


\title{
Group 10 Metal Dithiolene Bis(isonitrile) Complexes: Synthesis, Structures, Properties and Reactivity
}

\author{
Antony Obanda, ${ }^{\ddagger}$ Kendra Valerius,, Joel T. Mague, ${ }^{\ddagger}$ \\ Stephen Sproules, ${ }^{\S}$ and James P. Donahue ${ }^{\ddagger, *}$
}

${ }^{\ddagger}$ Dept. of Chemistry, Tulane University, 6400 Freret St., New Orleans, LA 70118-5698, U.S.A.

${ }^{\S}$ WestCHEM, School of Chemistry, University of Glasgow, Glasgow, G12 8QQ, United Kingdom 
Abstract. Reaction of $\left[\left(\mathrm{Ph}_{2} \mathrm{C}_{2} \mathrm{~S}_{2}\right)_{2} \mathrm{M}\right]\left(\mathrm{M}=\mathrm{Ni}^{2+}, \mathrm{Pd}^{2+}, \mathrm{Pt}^{2+}\right)$ with 2 eq of $\mathrm{RN} \equiv \mathrm{C}(\mathrm{R}=\mathrm{Me}(\mathbf{a})$, $\mathrm{Bn}(\mathbf{b}), \mathrm{Cy}(\mathbf{c}),{ }^{t} \mathrm{Bu}(\mathbf{d}), 1$-Ad (e), $\left.\mathrm{Ph}(\mathbf{f})\right)$ yields $\left[\left(\mathrm{Ph}_{2} \mathrm{C}_{2} \mathrm{~S}_{2}\right) \mathrm{M}(\mathrm{C} \equiv \mathrm{NR})_{2}\right]\left(\mathrm{M}=\mathrm{Ni}^{2+}, \mathbf{4 a}-\mathbf{4 f} ; \mathrm{M}^{2} \mathrm{Pd}^{2+}\right.$, 5a-5f; $\mathrm{M}=\mathrm{Pt}^{2+}, \mathbf{6 a - 6 f}$ ), which are air-stable and amenable to chromatographic purification. All members have been characterized crystallographically. Structurally, progressively greater planarity tends to be manifest as $\mathrm{M}$ varies from $\mathrm{Ni}$ to $\mathrm{Pt}$, and a modest decrease in the $\mathrm{C} \equiv \mathrm{N}$ bond length of coordinated $\mathrm{C} \equiv \mathrm{NR}$ appears in moving from Ni toward Pt. Vibrational spectroscopy $\left(\mathrm{CH}_{2} \mathrm{Cl}_{2}\right.$ solution) reveals $v_{\mathrm{C} \equiv \mathrm{N}}$ frequencies for $\left[\left(\mathrm{Ph}_{2} \mathrm{C}_{2} \mathrm{~S}_{2}\right) \mathrm{M}(\mathrm{C} \equiv \mathrm{NR})_{2}\right]$ that are substantially higher than for free $\mathrm{C} \equiv \mathrm{NR}$ and increase as $\mathrm{M}$ ranges from $\mathrm{Ni}$ to Pt. This trend is interpreted as arising from increasing positive charge at $\mathrm{M}$ that stabilizes the linear, charge-separated resonance form of the ligand over the bent form with lowered C-N bond order. UV-vis spectra reveal lowest energy transitions that are assigned as HOMO (dithiolene $\pi$ ) $\rightarrow$ LUMO (M-L $\sigma^{*}$ ) excitations. Oneelectron oxidations of $\left[\left(\mathrm{Ph}_{2} \mathrm{C}_{2} \mathrm{~S}_{2}\right) \mathrm{M}(\mathrm{C} \equiv \mathrm{NR})_{2}\right]$ are observed at $\sim+0.5 \mathrm{~V}$ due to $\mathrm{Ph}_{2} \mathrm{C}_{2} \mathrm{~S}_{2}{ }^{2-} \rightarrow \mathrm{Ph}_{2} \mathrm{C}_{2} \mathrm{~S}^{-}$ $\mathrm{S}^{\bullet}+\mathrm{e}^{-}$. Chemical oxidation of $\left[\left(\mathrm{Ph}_{2} \mathrm{C}_{2} \mathrm{~S}_{2}\right) \mathrm{Pt}\left(\mathrm{C} \equiv \mathrm{N}^{t} \mathrm{Bu}\right)_{2}\right]$ with $\left[\left(\mathrm{Br}-p-\mathrm{C}_{6} \mathrm{H}_{4}\right)_{3} \mathrm{~N}\right]\left[\mathrm{SbCl}_{6}\right]$ yields $\left[\left(\mathrm{Ph}_{2} \mathrm{C}_{2} \mathrm{~S}^{-} \mathrm{S}^{*}\right) \mathrm{Pt}\left(\mathrm{C} \equiv \mathrm{N}^{t} \mathrm{Bu}\right)_{2}\right]^{1+}$, identified spectroscopically, but in the crystalline state $\left[\left[\left(\mathrm{Ph}_{2} \mathrm{C}_{2} \mathrm{~S}^{-}\right.\right.\right.$ $\left.\left.\left.\mathrm{S}^{*}\right) \mathrm{Pt}\left(\mathrm{C} \equiv \mathrm{N}^{t} \mathrm{Bu}\right)_{2}\right]_{2}\right]^{2+}$ prevails, which forms via axial Pt $\cdots \mathrm{S}$ interactions and pyramidalization at metal. Complete substitution of MeNC from $\left[\left(\mathrm{Ph}_{2} \mathrm{C}_{2} \mathrm{~S}_{2}\right) \mathrm{Ni}(\mathrm{C} \equiv \mathrm{NMe})_{2}\right]$ by 2,6-Me 2 py under forcing conditions yields $\left[\left(2,6-\mathrm{Me}_{2} \mathrm{py}\right) \mathrm{Ni}\left(\mu_{2}-\eta^{1}, \eta^{1}-\mathrm{S}^{\prime}, \eta^{1}-\mathrm{S}^{\prime \prime}-\mathrm{C}_{2} \mathrm{Ph}_{2}\right)\right]_{2}$ (8), which features a folded $\mathrm{Ni}_{2} \mathrm{~S}_{2}$ core. In most cases, isonitrile substitution from $\left[\left(\mathrm{Ph}_{2} \mathrm{C}_{2} \mathrm{~S}_{2}\right) \mathrm{M}(\mathrm{C} \equiv \mathrm{NMe})_{2}\right]$ with monodentate ligands ( $\mathrm{L}=$ phosphine, $\mathrm{CN}^{-}$, carbene) typically leads to $\left[\left(\mathrm{Ph}_{2} \mathrm{C}_{2} \mathrm{~S}_{2}\right) \mathrm{M}(\mathrm{L})(\mathrm{C} \equiv \mathrm{NMe})\right]^{n}\left(n=0\right.$ or $\left.1^{-}\right)$, wherein $v_{\mathrm{C} \equiv \mathrm{N}}$ varies according to the relative $\sigma$ donating power of $\mathrm{L}(\mathbf{9}-\mathbf{2 1})$. Use of 1,3-bis(2,6diisopropylphenyl)imidazol-2-ylidene (IPr) provides [( $\left.\left.\mathrm{Ph}_{2} \mathrm{C}_{2} \mathrm{~S}_{2}\right) \mathrm{M}(\mathrm{IPr})(\mathrm{C} \equiv \mathrm{NMe})\right]$ for $\mathrm{M}=\mathrm{Ni}(\mathbf{1 8})$ or Pd (19), but for Pt, attack by IPr at the isonitrile carbon occurs to yield the unusual $\eta^{1}, \kappa \mathrm{C}$ ketenimine complex $\left[\left(\mathrm{Ph}_{2} \mathrm{C}_{2} \mathrm{~S}_{2}\right) \mathrm{Pt}(\mathrm{C}(\mathrm{NMe})(\mathrm{IPr}))(\mathrm{C} \equiv \mathrm{NMe})\right](\mathbf{2 0})$. 


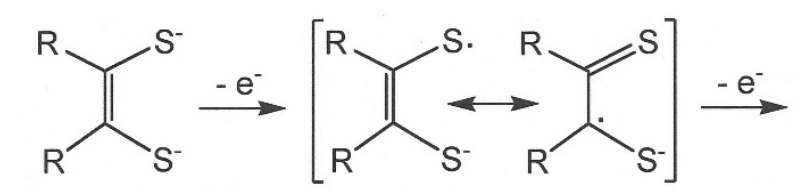

ene-1,2-dithiolate

(a) radical monoanion

(b)

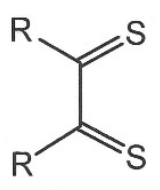

$\alpha$-dithione

(c)

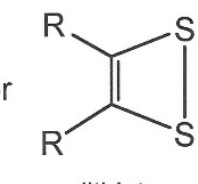

dithiete

(d)

Scheme 1. Redox levels of a dithiolene ligand.

\section{Introduction}

In their charge neutral state, the bis(dithiolene) complexes of the Group 10 metals are diamagnetic species comprised of a divalent metal ion and two dithiolene ligands in a radical monoanionic state, a redox level intermediate between fully reduced ene-1,2-dithiolate and fully oxidized $\alpha$-dithione or dithiete (Scheme 1). In the presence of a suitable reacting partner, these complexes undergo a ligand redox disproportionation whereby one of the two dithiolene ligands is extruded or transferred in an oxidized form, and the other ligand remains coordinated to $\mathrm{M}^{2+}$ as dithiolate dianion (Scheme 2). As shown, Scheme 2 is a conceptual formalism representing a partitioning of electrons that accounts for observed products, as opposed to being a discrete reaction step itself. This ligand redox disproportionation admits of two different perspectives. On one hand, $\left[\left(\mathrm{R}_{2} \mathrm{C}_{2} \mathrm{~S}_{2}\right)_{2} \mathrm{M}\right]$ complexes effectively operate as delivery agents of reactive oxidized dithiolene ligands that are otherwise not isolable and which can oxidatively add to a low-valent coordination complex, if present. The original and historically important example of this reactivity was synthesis by Schrauzer of $\left[\left(\mathrm{R}_{2} \mathrm{C}_{2} \mathrm{~S}_{2}\right)_{2} \mathrm{M}(\mathrm{CO})_{2}\right](\mathrm{M}=\mathrm{Mo}, \mathrm{W} ; \mathrm{R}=\mathrm{Ph}, \mathrm{Me}){ }^{1}$ These molecules have been of considerable usefulness as entry points toward small molecule analogues of the catalytic sites of molybdoand tungstoenzymes. ${ }^{2}$ Others, ${ }^{3-8}$ particularly Morris and coworkers, ${ }^{4-8}$ have effectively exploited this behavior for the preparation of a variety of new organometallic and inorganic transition metal complexes. In these examples, the value-added product is the coordination complex formed by oxidative addition of dithiolene ligand, while $\left[\left(\mathrm{R}_{2} \mathrm{C}_{2} \mathrm{~S}_{2}\right) \mathrm{M}\right]_{\mathrm{x}}$ is separated as an oligomeric or insoluble polymeric byproduct. On the other hand, if a suitable ligand $\mathrm{L}$ is introduced to $\left[\left(\mathrm{R}_{2} \mathrm{C}_{2} \mathrm{~S}_{2}\right)_{2} \mathrm{M}\right]$, well-defined new heteroleptic dithiolene complexes of the form $\left[\left(\mathrm{R}_{2} \mathrm{C}_{2} \mathrm{~S}_{2}\right) \mathrm{ML}_{2}\right]\left(\mathrm{L}_{2}=\right.$ two monodentate or one bidentate soft $\sigma$-donor $)$ are efficiently produced, while the expelled oxidized dithiolene ligand is separated as ill-defined oligomer or polymer. Here again, the original example of this reaction type was described by Schrauzer, who found it to be a direct and effective route toward $\left[\left(\mathrm{R}_{2} \mathrm{C}_{2} \mathrm{~S}_{2}\right) \mathrm{M}\left(\mathrm{PR}_{3}\right)_{2}\right]$ complexes. ${ }^{1,9}$<smiles></smiles><smiles>[R]C1=C([R])[SH]2CCN2S1</smiles><smiles>[R]C(=S)C([R])=S</smiles><smiles>[R]c1ssc1[R]</smiles>

Scheme 2. Dithiolene ligand redox disproportionation in Group 10 bis(dithiolene) complexes. This scheme is a formalism that rationalizes products formed in reactions with other metal complexes and is not a depiction of a discrete reaction. 

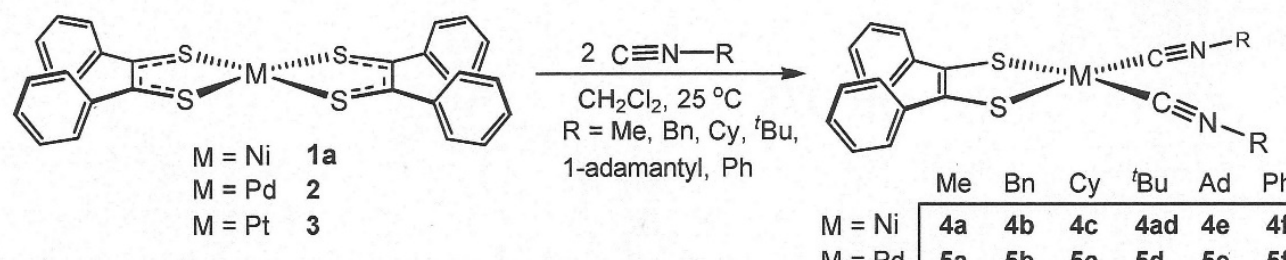

1-adamantyl, $\mathrm{Ph}$

\begin{tabular}{|c|c|c|c|c|c|c|}
\hline & $\mathrm{Me}$ & $\mathrm{Bn}$ & Cy & ${ }^{t} \mathrm{Bu}$ & $\mathrm{Ad}$ & $\mathrm{Ph}$ \\
\hline $\mathrm{M}=\mathrm{Ni}$ & $4 a$ & $4 b$ & $4 c$ & $4 a d$ & $4 e$ & $4 f$ \\
\hline $\mathrm{M}=\mathrm{Pd}$ & $5 a$ & $5 b$ & $5 c$ & $5 d$ & $5 e$ & $5 f$ \\
\hline$M=P t$ & $6 a$ & $6 b$ & $6 c$ & $6 d$ & $6 e$ & $6 f$ \\
\hline
\end{tabular}

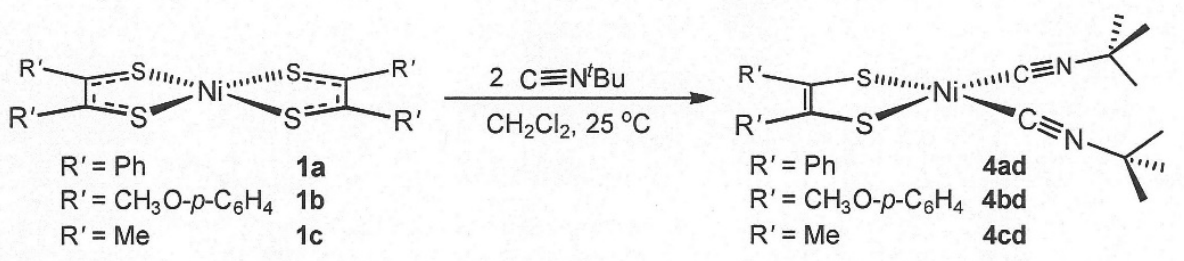

Scheme 3. Synthesis of Group 10 dithiolene bis(isonitrile) compounds.

As detailed in a recent account, ${ }^{10}$ we were motivated to survey the generality of dithiolene extrusion from $\left[\left(\mathrm{R}_{2} \mathrm{C}_{2} \mathrm{~S}_{2}\right)_{2} \mathrm{M}\right]$ to form $\left[\left(\mathrm{R}_{2} \mathrm{C}_{2} \mathrm{~S}_{2}\right) \mathrm{ML}_{2}\right]$ beyond the limited bounds of Schrauzer's work because of potential we saw for use of $\left[\left(\mathrm{R}_{2} \mathrm{C}_{2} \mathrm{~S}_{2}\right) \mathrm{ML}_{2}\right]$ compounds in the targeted synthesis of complex multi-metal dithiolene complexes. The synthesis of $\left[\left(\mathrm{Ph}_{2} \mathrm{C}_{2} \mathrm{~S}_{2}\right) \mathrm{M}(\mathrm{CNR})_{2}\right]\left(\mathrm{M}=\mathrm{Ni}^{2+}, \mathrm{Pd}^{2+}, \mathrm{Pt}^{2+} ; \mathrm{R}=\mathrm{Me}, \mathrm{Cy},{ }^{t} \mathrm{Bu}, 1-\mathrm{Ad}\right.$, $\mathrm{Ph}$ ) were disclosed in that report as well as a preliminary study of the further reactivity of $\left[\left(\mathrm{Ph}_{2} \mathrm{C}_{2} \mathrm{~S}_{2}\right) \mathrm{Ni}(\mathrm{CNMe})_{2}\right]$. In this following work, we more fully characterize the $\left[\left(\mathrm{Ph}_{2} \mathrm{C}_{2} \mathrm{~S}_{2}\right) \mathrm{M}(\mathrm{CNR})_{2}\right]$ compounds, especially structurally, and we more thoroughly lay out the panorama of outcomes when $\left[\left(\mathrm{Ph}_{2} \mathrm{C}_{2} \mathrm{~S}_{2}\right) \mathrm{M}(\mathrm{CNMe})_{2}\right]\left(\mathrm{M}=\mathrm{Ni}^{2+}, \mathrm{Pd}^{2+}, \mathrm{Pt}^{2+}\right)$ are introduced to new ligands intended to substitute for MeNC.

\section{Experimental}

Physical Methods: UV-vis spectra were obtained at ambient temperature with a Hewlett-Packard 8455a diode array spectrometer, while IR spectra were taken as $\mathrm{CH}_{2} \mathrm{Cl}_{2}$ solutions with a Thermo Nicolet Nexus 670 Fourier transform infrared instrument in absorption mode. All ${ }^{1} \mathrm{H},{ }^{13} \mathrm{C}$ and ${ }^{31} \mathrm{P}$ NMR spectra were recorded at $25^{\circ} \mathrm{C}$ with a Varian Unity Inova spectrometer operating at $400 \mathrm{MHz}$ or with a Bruker Avance spectrometer operating at $300 \mathrm{MHz}$. Both ${ }^{1} \mathrm{H}$ and ${ }^{13} \mathrm{C}$ NMR spectra were referenced to the solvent signal, while an external aqueous $\mathrm{H}_{3} \mathrm{PO}_{4}$ solution was employed as reference for all ${ }^{31} \mathrm{P}$ spectra. X-band EPR spectra were recorded on a Bruker ELEXSYS E500 spectrometer, while simulations were performed with XSophe ${ }^{11}$ distributed by Bruker Biospin GmbH. Mass spectrometry (MS) spectra (matrix-assisted laser desorption ionization - time of flight, MALDI-TOF) were obtained with a Bruker Autoflex III instrument operating in positive ion mode. Electrochemical measurements were made with a CHI 620C electroanalyzer workstation using a $\mathrm{Ag} / \mathrm{AgCl}$ reference electrode, a glassy carbon disk working electrode, a Pt wire auxiliary electrode, and $\left[{ }^{n} \mathrm{Bu}_{4} \mathrm{~N}\right]\left[\mathrm{PF}_{6}\right]$ as supporting electrolyte. Typical scan rates were 50 or 100 $\mathrm{mV} / \mathrm{sec}$. Under these conditions, the $\mathrm{Cp}_{2} \mathrm{Fe}^{+} / \mathrm{Cp}_{2} \mathrm{Fe}$ couple consistently occurred at $+540 \mathrm{mV}$. Elemental 
analyses were performed by Midwest Microlab, LLC (Indianapolis, IN), Galbraith Laboratories, Inc. (Knoxville, TN), or by Kolbe Microanalytical Laboratory (Oberhausen, Germany). Details regarding the collection of X-ray diffraction data and the solving and refining of crystal structures are deferred to supporting information.

Syntheses. The $\left[\left(\mathrm{Ph}_{2} \mathrm{C}_{2} \mathrm{~S}_{2}\right)_{2} \mathrm{M}\right],\left(\mathrm{M}=\mathrm{Ni}^{2+}, \mathrm{Pd}^{2+}, \mathrm{Pt}^{2+}\right)$ starting materials were synthesized following the procedure outlined by Schrauzer and Mayweg. ${ }^{1}$ Methyl isonitrile was made by the dehydration of $N$ methylformamide, ${ }^{12}$ and its density was experimentally determined to be $0.78 \mathrm{~g} / \mathrm{mL}$, while tert-butylisonitrile $(\mathrm{d} .=0.74 \mathrm{~g} / \mathrm{mL})$ and phenyl isonitrile $(\mathrm{d} .=0.98 \mathrm{~g} / \mathrm{mL})^{13}$ were synthesized via the phase-transfer Hofmann-carbylamine reaction. ${ }^{14}$ Cyclohexyl isonitrile (d. $\left.=0.878 \mathrm{~g} / \mathrm{mL}\right), 1$-adamantyl isonitrile, benzyl isonitrile (d. $=0.962 \mathrm{~g} / \mathrm{mL}$ ), and $\left[\mathrm{Et}_{4} \mathrm{~N}\right][\mathrm{CN}]$ were used as received from commercial sources. Tetraisopropylbenzobisimidazolium dibromide was prepared following a published procedure ${ }^{15}$ and deprotected by a modification of the protocol by Bantreil and Nolan. ${ }^{16}$ The $\left[\mathrm{BF}_{4}\right]^{1-}$ salt of $[\mathrm{IPr}-\mathrm{H}]^{+}(\mathrm{IPr}=$ 1,3-bis(2,6-diisopropylphenyl)imidazol-2-ylidene) was prepared and unmasked under $\mathrm{N}_{2}$ following the same protocol reported by Bantreil and Nolan. ${ }^{16}$ The free IPr ligand was stored in a glovebox. A published procedure was also implemented for the synthesis of $p$-phenylenebis(diphenylphosphine) (1,4-dppb). ${ }^{17}$ Column chromatography separations were carried out using $63-200 \mu \mathrm{m}$ silica (Dynamic Adsorbents) or 150 mesh neutral alumina (Aldrich). The solvents used for reactions were either dried with a system of drying columns from the Glass Contour Company $\left(\mathrm{CH}_{2} \mathrm{Cl}_{2}\right.$, THF), freshly distilled according to standard procedures ${ }^{18}(\mathrm{MeCN})$, or used as received from commercial suppliers. All reactions were conducted under an inert atmosphere of either $\mathrm{N}_{2}$ or Ar. Subsequent purification by chromatography and crystallization were done in the open air, except where noted otherwise.

[(Ph $\left.\left.\mathbf{C}_{2} \mathbf{S}_{2}\right) \mathbf{N i}(\mathbf{C N B n})_{2}\right]$, 4b. Benzyl isocyanide $(0.040 \mathrm{~mL}$, d. $=0.962 \mathrm{~g} / \mathrm{mL}, 0.33 \mathrm{mmol})$ was delivered dropwise via a gas-tight syringe to a solution of $\left[\left(\mathrm{Ph}_{2} \mathrm{C}_{2} \mathrm{~S}_{2}\right)_{2} \mathrm{Ni}\right](0.100 \mathrm{~g}, 0.184 \mathrm{mmol})$ in $\mathrm{CH}_{2} \mathrm{Cl}_{2}(20 \mathrm{~mL})$. The mixture was stirred at $25^{\circ} \mathrm{C}$ for $5 \mathrm{~h}$, after which time the solvent was removed under reduced pressure. The dark blue solid residual was applied directly to the top of a silica gel column packed as a slurry in hexanes. Flash elution with 1:1 $\mathrm{CH}_{2} \mathrm{Cl}_{2}$ :hexanes moved a green band of unreacted $\left[\left(\mathrm{Ph}_{2} \mathrm{C}_{2} \mathrm{~S}_{2}\right)_{2} \mathrm{Ni}\right]$ followed by a well resolved blue band of $\mathbf{4 b}$. This blue fraction was collected using 2:1 $\mathrm{CH}_{2} \mathrm{Cl}_{2}$ :hexanes and taken to dryness under reduced pressure. The solid residue was washed with $n$-pentane $(5 \mathrm{~mL})$, redissolved in a minimal volume of $\mathrm{CH}_{2} \mathrm{Cl}_{2}$, filtered through a Celite pad and taken to dryness again. Crystallization was accomplished by slow diffusion of hexanes vapor into a concentrated 1,2-dichloroethane solution. Yield: 0.089 g. $\mathrm{R}_{\mathrm{f}}=0.39$ (2:1 $\mathrm{CH}_{2} \mathrm{Cl}_{2}$ :hexanes). ${ }^{1} \mathrm{H} \mathrm{NMR}\left(\delta\right.$, ppm in $\left.\mathrm{CD}_{2} \mathrm{Cl}_{2}\right)$ : 7.46-7.35 (overlapping $\mathrm{m}, 10 \mathrm{H}, \mathrm{Bn}$, aromatic $\mathrm{C}-\mathrm{H}$ ), 7.19-7.09 (overlapping $\mathrm{m}, 10 \mathrm{H}$, aromatic $\mathrm{C}-\mathrm{H}$ ), $4.88\left(\mathrm{~s}, 4 \mathrm{H}, \mathrm{CH}_{2}\right) .{ }^{13} \mathrm{C} \mathrm{NMR}(\delta$, ppm in $\left.\mathrm{CD}_{2} \mathrm{Cl}_{2}\right): 142.1$, 138.6, 131.7, 130.1, 129.6, 129.3, 128.0, 127.2, 126.5, $48.7\left(\mathrm{CH}_{2}\right) . \mathrm{IR}\left(\mathrm{CH}_{2} \mathrm{Cl}_{2}, \mathrm{~cm}^{-1}\right): 2213$ (vs, $\mathrm{CN}_{\text {sym }}$ ), 2197 (vs, $\mathrm{CN}_{\text {asym }}$ ). UV-vis absorption spectrum $\left[\mathrm{CH}_{2} \mathrm{Cl}_{2}, \lambda_{\max }, \mathrm{nm}\left(\varepsilon_{\mathrm{M}}, \mathrm{M}^{-1} \mathrm{~cm}^{-1}\right)\right]$ : 460 (400), 
608 (420). Cyclic voltammetry (CV): $4 \mathbf{b}-\mathrm{e}^{-} \rightarrow[4 \mathbf{b}]^{1+},+0.50$ V. Anal. Calcd for $\mathrm{C}_{30} \mathrm{H}_{24} \mathrm{~N}_{2} \mathrm{~S}_{2} \mathrm{Ni}$ : C, 67.31; H, 4.52; N, 5.23. Found: C, 67.18; H, 4.67; N, 5.21.

$\left[\left(\mathrm{Ph}_{2} \mathrm{C}_{2} \mathrm{~S}_{2}\right) \mathbf{N i}(\mathrm{CNCy})_{2}\right], 4 \mathrm{c}$. The same procedures and scale used in the synthesis of $\mathbf{4 b}$ were employed but with $0.05 \mathrm{~mL}$ ( $0.40 \mathrm{mmol})$ of cyclohexylisonitrile. Ultimately, dark purple crystals of $\mathbf{4 c}$ were obtained. Yield: $0.082 \mathrm{~g}$, 95\%. $\mathrm{R}_{\mathrm{f}}=0.52$ (2:1 $\mathrm{CH}_{2} \mathrm{Cl}_{2}$ :hexanes). ${ }^{1} \mathrm{H}$ NMR ( $\delta$, ppm in $\mathrm{CD}_{2} \mathrm{Cl}_{2}$ ): 7.17-7.08 (overlapping m, 10H, aromatic C-H), 3.90 (m, 2H, Cy -CHNC), 1.94-1.89 (m, 4H, Cy), 1.81-1.73 (m, 8H, Сy), 1.541.46 (m, 8H, Cy). ${ }^{13} \mathrm{C}$ NMR ( $\delta$, ppm in $\mathrm{CD}_{2} \mathrm{Cl}_{2}$ ): 142.4, 138.4, $135.9(\mathrm{t}), 130.1,127.9,126.3,55.4(\mathrm{Cy}, \mathrm{CH})$, 32.4 (Cy, $\mathrm{CH}_{2}$ ), 25.2 (Cy, $\mathrm{CH}_{2}$ ), 22.9 (Cy, $\left.\mathrm{CH}_{2}\right)$. IR $\left(\mathrm{CH}_{2} \mathrm{Cl}_{2}, \mathrm{~cm}^{-1}\right.$ ): 2204 (vs, $\mathrm{CN}_{\text {sym }}$ ), 2189 (vs, $\mathrm{CN}_{\text {asym) }}$ ). UV-vis absorption spectrum $\left[\mathrm{CH}_{2} \mathrm{Cl}_{2}, \lambda_{\max }, \mathrm{nm}\left(\varepsilon_{\mathrm{M}}, \mathrm{M}^{-1} \mathrm{~cm}^{-1}\right)\right]: 440$ (240), 601 (420). MALDI MS. Calcd for $\mathrm{C}_{28} \mathrm{H}_{32} \mathrm{~N}_{2} \mathrm{~S}_{2} \mathrm{Ni}: \mathrm{m} / \mathrm{z}$ 519.401. Observed: $\mathrm{m} / \mathrm{z}$ 519.042. Cyclic voltammetry: 4c $-\mathrm{e}^{-} \rightarrow[4 \mathrm{c}]^{1+},+0.56 \mathrm{~V}$. Anal. Calcd for $\mathrm{C}_{28} \mathrm{H}_{32} \mathrm{~N}_{2} \mathrm{~S}_{2} \mathrm{Ni}$ : C, 64.74; H, 6.22; N, 5.39. Found: C, 64.86; H, 5.95; N, 5.43.

[(Ph $\left.\left.\mathbf{P h}_{2} \mathbf{S}_{2}\right) \mathbf{N i}\left(\mathbf{C N}^{t} \mathbf{B u}\right)_{2}\right]$, 4ad. $0.040 \mathrm{~mL}(\mathrm{~d} .=0.74 \mathrm{~g} / \mathrm{mL}, 0.36 \mathrm{mmol})$ of tert-butylisonitrile was delivered dropwise via a gas-tight syringe to a solution of $\mathbf{1 a}(0.100 \mathrm{~g}, 0.184 \mathrm{mmol})$, in $\mathrm{CH}_{2} \mathrm{Cl}_{2}(25 \mathrm{~mL})$ under an Ar atmosphere. Subsequent work-up and purification were as described for $\mathbf{4 b}$. Crystallization was accomplished by slow diffusion of $\mathrm{Et}_{2} \mathrm{O}$ vapor into a concentrated $\mathrm{CH}_{2} \mathrm{Cl}_{2}$ solution to ultimately give dark blue crystals of 4ad. Yield: $0.067 \mathrm{~g}, 81 \%$. $\mathrm{R}_{\mathrm{f}}=0.46\left(2: 1 \mathrm{CH}_{2} \mathrm{Cl}_{2}\right.$ :hexanes). ${ }^{1} \mathrm{H}$ NMR ( $\delta$, ppm in $\mathrm{CD}_{2} \mathrm{Cl}_{2}$ ): 7.16-7.14 (m, 4H, aromatic $\left.\mathrm{C}-\mathrm{H}\right), 7.12-7.09(\mathrm{~m}, 6 \mathrm{H}$, aromatic $\mathrm{C}-\mathrm{H}), 1.53\left(\mathrm{~s}, 18 \mathrm{H},{ }^{t} \mathrm{Bu}\right) .{ }^{13} \mathrm{C} \mathrm{NMR}$

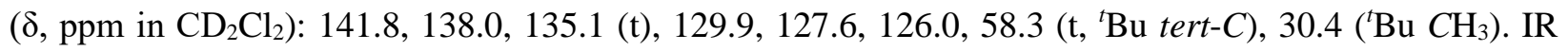
$\left(\mathrm{CH}_{2} \mathrm{Cl}_{2}, \mathrm{Cm}^{-1}\right)$ : 2197 (vs, $\left.\mathrm{CN}_{\text {sym }}\right), 2180$ (vs, $\left.\mathrm{CN}_{\text {asym }}\right)$. UV-Vis absorption spectrum $\left[\mathrm{CH}_{2} \mathrm{Cl}_{2 ;} \lambda_{\max }, \mathrm{nm}\left(\varepsilon_{\mathrm{M}}\right.\right.$,

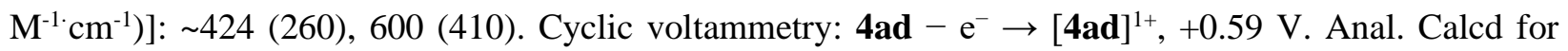
$\mathrm{C}_{24} \mathrm{H}_{28} \mathrm{~N}_{2} \mathrm{~S}_{2} \mathrm{Ni}$ : C, 61.68; H, 6.04; N, 5.99. Found: C, 61.91; H, 6.04; N, 6.08.

[( $\left.\left.\left.\mathbf{M e O}-\boldsymbol{p}-\mathbf{C}_{6} \mathbf{H}_{4}\right)_{2} \mathbf{C}_{2} \mathbf{S}_{2}\right) \mathbf{N i}\left(\mathbf{C N}^{t} \mathbf{B u}\right)_{2}\right]$, 4 bd. Two eq of tert-butylisonitrile $(0.03 \mathrm{~mL}$, d. = $0.74 \mathrm{~g} / \mathrm{mL}, 0.27$ mmol) were added via syringe to a solution of [(MeO- $\left.\left.\left.p-\mathrm{C}_{6} \mathrm{H}_{4}\right)_{2} \mathrm{C}_{2} \mathrm{~S}_{2}\right)_{2} \mathrm{Ni}\right](0.100 \mathrm{~g}, 0.151 \mathrm{mmol})$ in $\mathrm{CH}_{2} \mathrm{Cl}_{2}$ (25 mL). Subsequent steps in work-up and purification were analogous to those described for compound 4b; dark blue crystals of $4 \mathbf{b d}$ were ultimately obtained. Yield: 0.069 g, 98\%. $\mathrm{R}_{\mathrm{f}}=0.37$ (2:1 $\mathrm{CH}_{2} \mathrm{Cl}_{2}$ :hexanes). ${ }^{1} \mathrm{H}$ NMR ( $\delta$, ppm in $\left.\mathrm{CD}_{2} \mathrm{Cl}_{2}\right)$ : 7.11-7.06 (m, 2H, aromatic $\left.\mathrm{C}-\mathrm{H}\right)$, 6.69-6.64 (m, 2H, aromatic $\mathrm{C}-\mathrm{H}$ ), 3.73 (s, $\left.6 \mathrm{H},-\mathrm{OCH}_{3}\right), 1.53$ (s, $\left.18 \mathrm{H},{ }^{t} \mathrm{Bu}\right) .{ }^{13} \mathrm{C} \mathrm{NMR}\left(\delta, \mathrm{ppm}\right.$ in $\left.\mathrm{CDCl}_{3}\right): 157.9,136.8,134.9$, 131.1, 113.1, 58.2 ( ${ }^{t} \mathrm{Bu}$ tert- $\left.\mathrm{C}\right), 55.3\left(-\mathrm{OCH}_{3}\right), 30.3$ ( $\left.{ }^{t} \mathrm{Bu} \mathrm{CH}_{3}\right)$. IR $\left(\mathrm{CH}_{2} \mathrm{Cl}_{2}, \mathrm{~cm}^{-1}\right)$ : 2196 (vs, $\left.\mathrm{CN}_{\text {sym }}\right), 2180$ (vs, $\mathrm{CN}_{\text {asym }}$ ). UV-vis absorption spectrum $\left[\mathrm{CH}_{2} \mathrm{Cl}_{2}, \lambda_{\max }, \mathrm{nm}\left(\varepsilon_{\mathrm{M}}, \mathrm{M}^{-1} \mathrm{~cm}^{-1}\right)\right]$ : 420 (200), 607 (320). MALDI MS. Calcd for $\mathrm{C}_{26} \mathrm{H}_{32} \mathrm{~N}_{2} \mathrm{O}_{2} \mathrm{~S}_{2} \mathrm{Ni}: \mathrm{m} / \mathrm{z}$ 527.378. Observed: $\mathrm{m} / \mathrm{z}$ 527.071. Cyclic voltammetry: 4bd $-\mathrm{e}^{-} \rightarrow$ [4bd] ${ }^{1+}$, +0.50 V. Anal. Calcd for $\mathrm{C}_{26} \mathrm{H}_{32} \mathrm{~N}_{2} \mathrm{O}_{2} \mathrm{~S}_{2} \mathrm{Ni}$ : C, 59.21; H, 6.13; N, 5.31; S, 12.16. Found: C, 59.06; H, 6.05; N, 5.36; S, 12.01 .

[( $\left.\left(\mathbf{M e}_{2} \mathbf{C}_{2} \mathbf{S}_{2}\right) \mathbf{N i}\left(\mathbf{C N}^{t} \mathbf{B u}\right)_{2}\right]$, 4cd. Neat tert-butylisonitrile $(0.19 \mathrm{~mL}, 1.70 \mathrm{mmol})$ was delivered via syringe to a solution of $\left[\left(\mathrm{Me}_{2} \mathrm{C}_{2} \mathrm{~S}_{2}\right)_{2} \mathrm{Ni}\right](0.251 \mathrm{~g}, 0.85 \mathrm{mmol})$ in $\mathrm{CH}_{2} \mathrm{Cl}_{2}(25 \mathrm{~mL})$. The remaining synthesis and 
purification steps were analogous to those employed for compound $\mathbf{4 b}$. Crystallization was achieved by diffusing hexanes into a concentrated toluene solution of 4cd with a few drops of tert-butylisonitrile added in the inner vial. Yield: $0.086 \mathrm{~g}, 30 \%$. $\mathrm{R}_{\mathrm{f}}=0.37$ (2:1 $\mathrm{CH}_{2} \mathrm{Cl}_{2}$ :hexanes). ${ }^{1} \mathrm{H}$ NMR $\left(\delta\right.$, ppm in $\left.\mathrm{CD}_{2} \mathrm{Cl}_{2}\right): 1.95$ (s, 6H, $-\mathrm{CH}_{3}$ ), 1.50 (s, $\left.18 \mathrm{H},{ }^{t} \mathrm{Bu}\right) .{ }^{13} \mathrm{C}$ NMR ( $\delta$, ppm in $\mathrm{CDCl}_{3}$ ): 135.8, 130.6, $58.4\left({ }^{t} \mathrm{Bu}\right.$ tert-C), 30.4 (isonitrile $-\mathrm{CH}_{3}$ ), 20.7 (dithiolene $-\mathrm{CH}_{3}$ ). IR $\left(\mathrm{CH}_{2} \mathrm{Cl}_{2}, \mathrm{~cm}^{-1}\right.$ ): 2196 (vs, $\mathrm{CN}_{\text {sym }}$ ), 2177 (vs, $\mathrm{CN}_{\text {asym }}$ ). UV-vis absorption spectrum $\left[\mathrm{CH}_{2} \mathrm{Cl}_{2}, \lambda_{\max }, \mathrm{nm}\left(\varepsilon_{\mathrm{M}}, \mathrm{M}^{-1} \cdot \mathrm{cm}^{-1}\right)\right]$ : 350 (6400), 450 (290), 614 (170). Cyclic voltammetry: 4cd $-\mathrm{e}^{-} \rightarrow[\mathbf{4 c d}]^{1+},+0.42 \mathrm{~V}$.

[(Ph $\left.\left.\mathbf{C}_{2} \mathbf{S}_{2}\right) \mathbf{N i}(\mathbf{C N}-1-\mathbf{A d})_{2}\right]$, 4e. Solid 1-adamantylisonitrile (0.054 g, $\left.0.331 \mathrm{mmol}\right)$ was added to a solution of 1 (0.090 mg, $0.166 \mathrm{mmol})$ in $\mathrm{CH}_{2} \mathrm{Cl}_{2}(25 \mathrm{~mL})$. Subsequent steps in workup and purification procedure were similar to those implemented for $\mathbf{4 b}$, ultimately yielding dark blue crystals of $\mathbf{4 e}$. Yield: 0.076 g, $74 \%$. $\mathrm{R}_{\mathrm{f}}=0.64$ (2:1 $\mathrm{CH}_{2} \mathrm{Cl}_{2}$ :hexanes). ${ }^{1} \mathrm{H}$ NMR ( $\delta$, ppm in $\mathrm{CD}_{2} \mathrm{Cl}_{2}$ ): 7.16-7.08 (overlapping $\mathrm{m}$, $10 \mathrm{H}$, aromatic $\mathrm{C}-\mathrm{H}), 2.13(\mathrm{~m}, 6 \mathrm{H}$, adamantly $\mathrm{C}-\mathrm{H}), 2.10(\mathrm{~m}, 12 \mathrm{H}$, adamantyl $\mathrm{C}-\mathrm{H}), 1.70(\mathrm{~m}, 12 \mathrm{H}$, adamantyl $\mathrm{C}-H) .{ }^{13} \mathrm{C}$ NMR $\left(\delta\right.$, ppm in $\mathrm{CD}_{2} \mathrm{Cl}_{2}$ ): 142.4, 138.4, $134.5(\mathrm{t}), 130.1,127.9,126.3$, 58.6, 43.3, 35.6, 29.3. IR ( $\left.\mathrm{CH}_{2} \mathrm{Cl}_{2}, \mathrm{~cm}^{-1}\right)$ : 2196 (vs, $\mathrm{CN}_{\text {sym }}$ ), 2175 (vs, $\mathrm{CN}_{\text {asym }}$ ). UV-vis absorption spectrum $\left[\mathrm{CH}_{2} \mathrm{Cl}_{2}\right.$, $\left.\lambda_{\max }, \mathrm{nm}\left(\varepsilon_{\mathrm{M}}, \mathrm{M}^{-1} \cdot \mathrm{cm}^{-1}\right)\right]: 437$ (180), 599 (360). Cyclic voltammetry: $4 \mathbf{e}-\mathrm{e}^{-} \rightarrow[\mathbf{4 e}]^{1+},+0.57 \mathrm{~V}$. Anal. Calcd for $\mathrm{C}_{36} \mathrm{H}_{40} \mathrm{~N}_{2} \mathrm{~S}_{2} \mathrm{Ni}$ : C, 69.34; H, 6.47; N, 4.49. Found: C, 69.22; H, 6.33; N, 4.54.

$\left[\left(\mathbf{P h}_{2} \mathbf{C}_{2} \mathbf{S}_{2}\right) \mathbf{N i}(\mathbf{C N P h})_{2}\right]$, $4 \mathbf{f}$. The procedure followed for the synthesis of $\mathbf{4 b}$ was employed using 0.080 $\mathrm{mL}(0.76 \mathrm{mmol})$ of phenylisonitrile, which was added to a solution of $\left[\left(\mathrm{Ph}_{2} \mathrm{C}_{2} \mathrm{~S}_{2}\right){ }_{2} \mathrm{Ni}\right](0.220 \mathrm{~g}, 0.405 \mathrm{mmol})$ in $\mathrm{CH}_{2} \mathrm{Cl}_{2}(25 \mathrm{~mL})$. Subsequent work-up and purification steps were the same as for $\mathbf{4 b}$. Diffraction-quality green crystals of $\mathbf{4 f}$ were obtained upon recrystallization. Yield: $0.143 \mathrm{~g}, 70 \%$. $\mathrm{R}_{\mathrm{f}}=0.63$ (2:1 $\mathrm{CH}_{2} \mathrm{Cl}_{2}$ :hexanes). ${ }^{1} \mathrm{H}$ NMR ( $\delta$, ppm in $\mathrm{CD}_{2} \mathrm{Cl}_{2}$ ): 7.53-7.48 (overlapping $\mathrm{m}, 10 \mathrm{H}$, aromatic $\mathrm{C}-H$ ), 7.23-7.21 $(\mathrm{m}, 4 \mathrm{H}$, aromatic $\mathrm{C}-\mathrm{H})$ 7.16-7.13 (m, 6H, aromatic $\mathrm{C}-\mathrm{H}) .{ }^{13} \mathrm{C} \mathrm{NMR}\left(\delta\right.$, ppm in $\left.\mathrm{CD}_{2} \mathrm{Cl}_{2}\right)$ : 142.0, 139.2, 130.8, 130.2, 128.0, 126.9, 126.7. IR $\left(\mathrm{CH}_{2} \mathrm{Cl}_{2}, \mathrm{~cm}^{-1}\right)$ : 2182 (vs, $\left.\mathrm{CN}_{\text {sym }}\right), 2162$ (vs, $\mathrm{CN}_{\text {asym}}$ ). UV-vis absorption spectrum $\left[\mathrm{CH}_{2} \mathrm{Cl}_{2}, \lambda_{\max }, \mathrm{nm}\left(\varepsilon_{\mathrm{M}}, \mathrm{M}^{-1} \cdot \mathrm{cm}^{-1}\right)\right]$ : 466 (1400), 639 (480). MALDI MS. Calcd for $\mathrm{C}_{28} \mathrm{H}_{20} \mathrm{~N}_{2} \mathrm{~S}_{2} \mathrm{Ni}: \mathrm{m} / \mathrm{z}$ 507.306. Observed: $\mathrm{m} / \mathrm{z}$ 507.003. Cyclic voltammetry: $4 \mathbf{f}-\mathrm{e}^{-} \rightarrow[\mathbf{4 f}]^{1+},+0.62 \mathrm{~V}$. Anal. Calcd for $\mathrm{C}_{28} \mathrm{H}_{20} \mathrm{~N}_{2} \mathrm{~S}_{2} \mathrm{Ni}$ : C, 69.29; H, 3.97; N, 5.52. Found: C, 66.26; H, 3.87; N, 5.56.

$\left[\left(\mathbf{P h}_{2} \mathbf{C}_{2} \mathbf{S}_{2}\right) \mathbf{P d}(\mathbf{C N M e})_{2}\right], 5 a$. The same procedures and scale used in the synthesis of $\mathbf{4 b}$ were employed but with 2 and $0.020 \mathrm{~mL}(0.38 \mathrm{mmol})$ of methylisonitrile. Ultimately, reddish-orange diffraction quality crystals of 5a were obtained. Yield: $0.050 \mathrm{~g}, 78 \%$. $\mathrm{R}_{\mathrm{f}}=0.11\left(2: 1 \mathrm{CH}_{2} \mathrm{Cl}_{2}\right.$ :hexanes). ${ }^{1} \mathrm{H}$ NMR ( $\delta$, ppm in $\mathrm{CD}_{2} \mathrm{Cl}_{2}$ ): 7.16-7.08 (overlapping $\mathrm{m}, 10 \mathrm{H}$, aromatic $\mathrm{C}-\mathrm{H}$ ), 3.45 (s, $\left.6 \mathrm{H}, \mathrm{CNCH}_{3}\right) .{ }^{13} \mathrm{C} \mathrm{NMR}(\delta$, ppm in $\mathrm{CD}_{2} \mathrm{Cl}_{2}$ ): 145.7, 137.3, 132.7, 130.8, 129.2, 33.4 (isonitrile - $\left.\mathrm{CH}_{3}\right)$. IR $\left(\mathrm{CH}_{2} \mathrm{Cl}_{2}, \mathrm{~cm}^{-1}\right)$ : 2246 (vs, $\mathrm{CN}_{\text {sym }}$ ), 2230 (vs, $\mathrm{CN}_{\text {asym }}$ ). UV-vis absorption spectrum $\left[\mathrm{CH}_{2} \mathrm{Cl}_{2}, \lambda_{\max }, \mathrm{nm}\left(\varepsilon_{\mathrm{M}}, \mathrm{M}^{-1} \cdot \mathrm{cm}^{-1}\right)\right]: 421$ (690), 502 (530). Cyclic voltammetry: 5a $-\mathrm{e}^{-} \rightarrow[5 \mathbf{5 a}]^{1+},+0.61$ V. Anal. Calcd for $\mathrm{C}_{18} \mathrm{H}_{16} \mathrm{~N}_{2} \mathrm{~S}_{2} \mathrm{Pd}$ : C, 50.17; H, 3.75; N, 6.50. Found: C, 49.12; H, 3.86; N, 6.48. 
$\left[\left(\mathbf{P h}_{2} \mathbf{C}_{2} \mathrm{~S}_{2}\right) \mathbf{P d}(\mathbf{C N B n})_{2}\right], 5 b$. The same procedures and scale used in the synthesis and purification of 4b were employed with 2 and $0.040 \mathrm{~mL}(0.33 \mathrm{mmol})$ of benzylisonitrile. Diffraction-quality red crystals of 5b were obtained. Yield: 0.057 g, $60 \% . \mathrm{R}_{\mathbf{f}}=0.35$ (2:1 $\mathrm{CH}_{2} \mathrm{Cl}_{2}$ :hexanes). ${ }^{1} \mathrm{H}$ NMR $\left(\delta\right.$, ppm in $\left.\mathrm{CD}_{2} \mathrm{Cl}_{2}\right)$ : 7.45-7.36 (overlapping $\mathrm{m}, 10 \mathrm{H}, \mathrm{Bn}$ aromatic $\mathrm{C}-\mathrm{H}$ ), 7.17-7.07 (overlapping $\mathrm{m}, 10 \mathrm{H}$, aromatic $\mathrm{C}-\mathrm{H}$ ), 4.92 (s, 4H, $\left.-\mathrm{CH}_{2}-\right) .{ }^{13} \mathrm{C} \mathrm{NMR}\left(\delta\right.$, ppm in $\left.\mathrm{CD}_{2} \mathrm{Cl}_{2}\right)$ : 143.1, 135.1, 131.3, 130.3, 129.7, 129.4, 127.9, 127.3, 126.4, $48.5\left(-\mathrm{CH}_{2} \mathrm{C}_{6} \mathrm{H}_{5}\right)$. Solution IR $\left(\mathrm{CH}_{2} \mathrm{Cl}_{2}, \mathrm{~cm}^{-1}\right)$ : 2227 (vs, $\mathrm{CN}_{\text {sym }}$ ), 2213 (vs, $\mathrm{CN}_{\text {asym }}$ ). UV-Vis absorption spectrum $\left[\mathrm{CH}_{2} \mathrm{Cl}_{2}, \lambda_{\max }, \mathrm{nm}\left(\varepsilon_{\mathrm{M}}, \mathrm{M}^{-1} \cdot \mathrm{cm}^{-1}\right)\right]$ : 427 (150), 518 (50). MALDI MS. Calcd for $\mathrm{C}_{30} \mathrm{H}_{24} \mathrm{~N}_{2} \mathrm{~S}_{2} \mathrm{Pd}: \mathrm{m} / \mathrm{z}$ 583.086. Observed: $m / z$ 582.733. Cyclic voltammetry: $5 \mathbf{b}-\mathrm{e}^{-} \rightarrow[5 \mathbf{b}]^{1+},+0.56 \mathrm{~V}$. Anal. Calcd for $\mathrm{C}_{30} \mathrm{H}_{24} \mathrm{~N}_{2} \mathrm{~S}_{2} \mathrm{Pd}$ : C, 61.80; H, 4.15; N, 4.80; S, 11.00. Found: C, 61.20; H, 4.06; N, 4.75; S, 10.62.

[(Ph $\left.\left.\mathbf{P}_{2} \mathbf{S}_{2}\right) \mathbf{P d}(\mathbf{C N C y})_{2}\right], 5$ c. $0.040 \mathrm{~mL}(0.32 \mathrm{mmol})$ of cyclohexylisonitrile was added to a solution of 2 (0.087 g, $0.147 \mathrm{mmol})$ in $\mathrm{CH}_{2} \mathrm{Cl}_{2}(25 \mathrm{~mL})$. The remaining synthesis and purification steps were analogous to those used for $\mathbf{4 b}$; ultimately, red crystals of $\mathbf{5 c}$ were produced. Yield: $0.067 \mathrm{~g}, 73 \%$. $\mathrm{R}_{\mathrm{f}}=0.51(2: 1$ $\mathrm{CH}_{2} \mathrm{Cl}_{2}$ :hexanes). ${ }^{1} \mathrm{H}$ NMR ( $\delta$, ppm in $\mathrm{CD}_{2} \mathrm{Cl}_{2}$ ): 7.17-7.07 (overlapping $\mathrm{m}, 10 \mathrm{H}$, aromatic $\mathrm{C}-\mathrm{H}$ ), 3.96 (br m, 2H, Cy), 1.93 (m, 4H, Cy) 1.85-1.78 (overlapping m, 8H, Cy), 1.45-1.50 (m, 8H, Cy). ${ }^{13} \mathrm{C} \mathrm{NMR}(\delta$, ppm in $\left.\mathrm{CD}_{2} \mathrm{Cl}_{2}\right)$ : 143.3, 135.0, 130.3, 127.8, 126.3, 55.3, 32.3, 25.2, 22.9. IR $\left(\mathrm{CH}_{2} \mathrm{Cl}_{2}, \mathrm{~cm}^{-1}\right): 2217$ (vs, $\mathrm{CN}_{\text {sym }}$ ), 2203 (vs, $\mathrm{CN}_{\text {asym }}$ ). UV-vis absorption spectrum $\left[\mathrm{CH}_{2} \mathrm{Cl}_{2} ; \lambda_{\max }, \mathrm{nm}\left(\varepsilon_{\mathrm{M}}, \mathrm{M}^{-1} \cdot \mathrm{cm}^{-1}\right)\right]: 431$ (430), 504 (490). Cyclic voltammetry (CV): 5c $-\mathrm{e}^{-} \rightarrow[5 \mathrm{c}]^{1+},+0.61$ V. Anal. Calcd for $\mathrm{C}_{28} \mathrm{H}_{32} \mathrm{~N}_{2} \mathrm{~S}_{2} \mathrm{Pd}$ : C, 59.30; $\mathrm{H}$, 5.69; N, 4.94. Found: C, 59.47; H, 5.99; N, 4.88.

[( $\left.\left(\mathbf{P h}_{2} \mathbf{C}_{2} \mathbf{S}_{2}\right) \mathbf{P d}(\mathbf{C N}-1-A d)_{2}\right]$, 5e. The same procedures and scale used in the synthesis of $\mathbf{4 b}$ were employed with 2 and $0.055 \mathrm{~g}$ (0.34 mmol) of 1-adamantylisonitrile. Diffraction-quality, red crystals of 5e were obtained. Yield: 0.062 g, 55\%. $\mathrm{R}_{\mathbf{f}}=0.61$ (2:1 $\mathrm{CH}_{2} \mathrm{Cl}_{2}$ :hexanes). ${ }^{1} \mathrm{H}$ NMR $\left(\delta\right.$, ppm in $\mathrm{CD}_{2} \mathrm{Cl}_{2}$ ): 7.17-7.07 (overlapping $\mathrm{m}, 10 \mathrm{H}$, aromatic $\mathrm{C}-\mathrm{H}$ ), 2.13 (overlapping $\mathrm{m}, 18 \mathrm{H}, \mathrm{Ad}$ ), 1.71 (overlapping $\mathrm{m}, 12 \mathrm{H}$, Ad). ${ }^{13} \mathrm{C}$ NMR ( $\delta$, ppm in $\left.\mathrm{CD}_{2} \mathrm{Cl}_{2}\right): 143.4,135.0,130.3,127.8,126.3$, 58.7, 43.2, 36.0, 29.3. $\mathrm{IR}\left(\mathrm{CH}_{2} \mathrm{Cl}_{2}\right.$, $\mathrm{cm}^{-1}$ ): 2211 (vs, $\mathrm{CN}_{\text {sym }}$ ), 2198 (vs, $\mathrm{CN}_{\text {asym }}$ ). UV-vis absorption spectrum $\left[\mathrm{CH}_{2} \mathrm{Cl}_{2} ; \lambda_{\max }, \mathrm{nm}\left(\varepsilon_{\mathrm{M}}, \mathrm{M}^{-1} \cdot \mathrm{cm}^{-1}\right)\right]$ : 430 (320), 505 (420). Cyclic voltammetry: $5 \mathbf{e}-\mathrm{e}^{-} \rightarrow[5 \mathbf{e}]^{1+},+0.59 \mathrm{~V}$. Anal. Calcd for $\mathrm{C}_{36} \mathrm{H}_{40} \mathrm{~N}_{2} \mathrm{~S}_{2} \mathrm{Pd}: \mathrm{C}$, 64.41; H, 6.01; N, 4.17. Found: C, 61.86; H, 6.08; N, 3.69.

[(Ph $\left.\left.\mathbf{C}_{2} \mathbf{S}_{2}\right) \mathbf{P d}(\mathbf{C N P h})_{2}\right]$, 5f. Phenylisonitrile $(0.040 \mathrm{~mL}, 0.38 \mathrm{mmol})$ was added to a solution of $\mathbf{2}(0.120$ g, $0.203 \mathrm{mmol})$, in $\mathrm{CH}_{2} \mathrm{Cl}_{2}(25 \mathrm{~mL})$. The remaining synthesis and purification steps were analogous to those implemented for compound $\mathbf{4 b}$, yielding red crystals of $\mathbf{5 f}$. Yield: 0.070 g, 66\%. $\mathrm{R}_{\mathrm{f}}=0.59(2: 1$ $\mathrm{CH}_{2} \mathrm{Cl}_{2}$ :hexanes). ${ }^{1} \mathrm{H}$ NMR ( $\delta$, ppm in $\mathrm{CD}_{2} \mathrm{Cl}_{2}$ ): 7.58-7.48 (overlapping $\mathrm{m}, 10 \mathrm{H}$, aromatic $\mathrm{C}-\mathrm{H}$ ), 7.21-7.19 (m, 4H, aromatic $\mathrm{C}-\mathrm{H})$, 7.14-7.11 (m, 6H, aromatic $\mathrm{C}-\mathrm{H})$. IR $\left(\mathrm{CH}_{2} \mathrm{Cl}_{2}, \mathrm{~cm}^{-1}\right): 2198$ (vs, $\left.\mathrm{CN}_{\mathrm{sym}}\right), 2182$ (vs, $\left.\mathrm{CN}_{\text {asym }}\right)$. UV-vis absorption spectrum $\left[\mathrm{CH}_{2} \mathrm{Cl}_{2}, \lambda_{\max }, \mathrm{nm}\left(\varepsilon_{\mathrm{M}}, \mathrm{M}^{-1} \cdot \mathrm{Cm}^{-1}\right)\right]: \sim 385$ (5180), $\sim 534$ (770). Cyclic voltammetry: $5 \mathbf{f}-\mathrm{e}^{-} \rightarrow[5 \mathbf{f}]^{1+},+0.67 \mathrm{~V}$. 
[(Ph $\left.\left.\mathbf{P}_{2} \mathrm{~S}_{2}\right) \mathbf{P t}(\mathrm{CNMe})_{2}\right], 6 a$. The same procedures and scale used in the synthesis and purification of 4b were employed but with 3 and methylisonitrile $(0.020 \mathrm{~mL}, 0.38 \mathrm{mmol})$. Ultimately, yellow crystals were obtained upon recrystallization by diffusion of $\mathrm{Et}_{2} \mathrm{O}$ into a concentrated $\mathrm{CH}_{2} \mathrm{Cl}_{2}$ solution of $\mathbf{6 a}$. Yield: 0.032 g, 42\%. $\mathrm{R}_{\mathbf{f}}=0.14\left(2: 1 \mathrm{CH}_{2} \mathrm{Cl}_{2}\right.$ :hexanes). ${ }^{1} \mathrm{H}$ NMR ( $\delta$, ppm in $\left.\mathrm{CD}_{2} \mathrm{Cl}_{2}\right): 7.19-7.09$ (unresolved m, $10 \mathrm{H}$, aromatic $\mathrm{C}-\mathrm{H}$ ), 3.42 (s, $\left.6 \mathrm{H}, \mathrm{CNCH}_{3}\right) .{ }^{13} \mathrm{C} \mathrm{NMR}\left(\delta\right.$, ppm in $\mathrm{CD}_{2} \mathrm{Cl}_{2}$ ): 142.9, 135.2, 130.4, 127.9, 126.4, 30.5 (isonitrile $-\mathrm{CH}_{3}$ ). IR $\left(\mathrm{CH}_{2} \mathrm{Cl}_{2}, \mathrm{~cm}^{-1}\right)$ : 2248 (vs, $\mathrm{CN}_{\text {sym }}$ ), 2223 (vs, $\mathrm{CN}_{\text {asym }}$ ). UV-vis absorption spectrum $\left[\mathrm{CH}_{2} \mathrm{Cl}_{2}, \lambda_{\max }, \mathrm{nm}\left(\varepsilon_{\mathrm{M}}, \mathrm{M}^{-1} \cdot \mathrm{cm}^{-1}\right)\right]: 337$ (4780). MALDI MS. Calcd for $\mathrm{C}_{18} \mathrm{H}_{16} \mathrm{~N}_{2} \mathrm{~S} 2 \mathrm{Pt}: \mathrm{m} / \mathrm{z} 519.554$. Observed: $m / z$ 519.858. Cyclic voltammetry: 6a $-\mathrm{e}^{-} \rightarrow[6 \mathbf{6 a}]^{1+},+0.64 \mathrm{~V}$. Anal. Calcd for $\mathrm{C}_{18} \mathrm{H}_{16} \mathrm{~N}_{2} \mathrm{~S}_{2} \mathrm{Pt}: \mathrm{C}$, 41.61; H, 3.11; N, 5.39. Found: C, 41.54; H, 2.92; N, 5.44.

[(Ph $\left.\left.\mathbf{P h}_{2} \mathbf{S}_{2}\right) \mathbf{P t}(\mathbf{C N B n})_{2}\right], 6 \mathbf{b}$. $0.070 \mathrm{~mL}(0.58 \mathrm{mmol})$ of benzylisonitrile was added to a solution of $\mathbf{3}$ (0.200 g, $0.294 \mathrm{mmol}$ ) in $\mathrm{CH}_{2} \mathrm{Cl}_{2}(20 \mathrm{~mL})$. The rest of the synthesis and purification procedures were analogous to those used for compound $\mathbf{4 b}$, yielding yellow crystals of $\mathbf{6 b}$. Yield: $0.073 \mathrm{~g}, 38 \%$. $\mathrm{R}_{\mathrm{f}}-0.35$ (2:1 $\mathrm{CH}_{2} \mathrm{Cl}_{2}$ :hexanes). ${ }^{1} \mathrm{H}$ NMR ( $\delta$, ppm in $\mathrm{CD}_{2} \mathrm{Cl}_{2}$ ): 7.50-7.40 (overlapping $\mathrm{m}, 10 \mathrm{H}$, aromatic $\mathrm{C}-\mathrm{H}$ ), 7.23-7.12 (overlapping m, 10H, aromatic $\mathrm{C}-\mathrm{H}$ ), 4.98 (s, $\left.4 \mathrm{H},-\mathrm{CH}_{2} \mathrm{C}_{6} \mathrm{H}_{5}\right) .{ }^{13} \mathrm{C} \mathrm{NMR}\left(\delta\right.$, ppm in $\mathrm{CD}_{2} \mathrm{Cl}_{2}$ ): 142.9, 135.4, 131.5, 130.5, 129.7, 129.4, 127.9, 127.3, 126.5, $48.8\left(-\mathrm{CH}_{2} \mathrm{C}_{6} \mathrm{H}_{5}\right) . \mathrm{IR}\left(\mathrm{CH}_{2} \mathrm{Cl}_{2}, \mathrm{~cm}^{-1}\right): 2229$ (vs, $\mathrm{CN}_{\text {sym }}$ ), 2204 (vs, $\mathrm{CN}_{\text {asym }}$ ). UV-vis absorption spectrum $\left[\mathrm{CH}_{2} \mathrm{Cl}_{2}, \lambda_{\max }, \mathrm{nm}\left(\varepsilon_{\mathrm{M}}, \mathrm{M}^{-1} \mathrm{~cm}^{-1}\right)\right]$ : 342 (1560). Cyclic voltammetry: $6 \mathbf{b}-\mathrm{e}^{-} \rightarrow[6 \mathbf{6 b}]^{1+},+0.57 \mathrm{~V}$. Anal. Calcd for $\mathrm{C}_{30} \mathrm{H}_{24} \mathrm{~N}_{2} \mathrm{~S}_{2} \mathrm{Pt}$ : C, 53.64; H, 3.61; N, 4.17; S, 9.54. Found: C, 53.49; H, 3.53; N, 4.13; S, 9.36.

[(Ph $\left.\left.\mathbf{P h}_{2} \mathbf{S}_{2}\right) \mathbf{P t}(\mathbf{C N C y})_{2}\right]$, 6c. Cyclohexylisonitrile $(0.070 \mathrm{~mL}, 0.56 \mathrm{mmol})$ of cyclohexylisonitrile was added to a solution of $3(0.203 \mathrm{~g}, 0.299 \mathrm{mmol})$ in $\mathrm{CH}_{2} \mathrm{Cl}_{2}(25 \mathrm{~mL})$. The remaining synthesis and purification steps were analogous to those used for compound $\mathbf{4 b}$, yielding yellow crystals of $\mathbf{6 c}$. Yield: $0.084 \mathrm{~g}, 46 \%$. $\mathrm{R}_{\mathrm{f}}=0.47$ (2:1 CH $\mathrm{CH}_{2} \mathrm{Cl}$ :hexanes). ${ }^{1} \mathrm{H}$ NMR ( $\delta$, ppm in $\mathrm{CD}_{2} \mathrm{Cl}_{2}$ ): 7.19-7.08 (overlapping $\mathrm{m}, 10 \mathrm{H}$, aromatic $\mathrm{C}-\mathrm{H}$ ), 4.02-3.98 (m, 2H, Cy), 1.97-1.93 (m, 4H, Cy), 1.90-1.75 (overlapping m, 8H, Cy), 1.51-1.46 (m, $8 \mathrm{H}$, Cy). ${ }^{13} \mathrm{C}$ NMR $\left(\delta\right.$, ppm in $\left.\mathrm{CD}_{2} \mathrm{Cl}_{2}\right): 143.1,135.3,130.5,127.8,126.3,56.0$, 32.3, 25.2, 23.0. $\mathrm{IR}\left(\mathrm{CH}_{2} \mathrm{Cl}_{2}\right.$, $\mathrm{cm}^{-1}$ ): 2219 (vs, $\mathrm{CN}_{\text {sym }}$ ), 2196 (vs, $\mathrm{CN}_{\text {asym }}$ ). UV-vis absorption spectrum $\left[\mathrm{CH}_{2} \mathrm{Cl}_{2}, \lambda_{\max }, \mathrm{nm}\left(\varepsilon_{\mathrm{M}}, \mathrm{M}^{-1} \cdot \mathrm{cm}^{-1}\right)\right]$ : 336 (9970). Cyclic voltammetry: 9c $-\mathrm{e}^{-} \rightarrow[9 \mathbf{9 c}]^{1+}$, +0.67 V. Anal. Calcd for $\mathrm{C}_{26} \mathrm{H}_{32} \mathrm{~N}_{2} \mathrm{~S}_{2} \mathrm{Pt}$ : C, 51.27; H, 4.93; N, 4.27. Found: C, 51.19; H, 4.83; N, 4.23.

[(Ph $\left.\left.\mathbf{P h}_{2} \mathrm{~S}_{2}\right) \mathbf{P t}\left(\mathrm{CN}^{t} \mathbf{B u}\right)_{2}\right], \mathbf{6 d}$. The same synthesis and purification procedures used in the preparation of 4b were employed but with $0.033 \mathrm{~mL}(0.29 \mathrm{mmol})$ of tert-butylisonitrile and $0.100 \mathrm{~g}(0.147 \mathrm{mmol})$ of 3 . Ultimately, yellow crystals of $\mathbf{6 d}$ were obtained upon recrystallization by diffusion of hexanes vapor into a 1,2-dichloroethane. Yield: 0.036 g, 41\%. $\mathrm{R}_{\mathbf{f}}=0.40$ (2:1 $\mathrm{CH}_{2} \mathrm{Cl}_{2}$ :hexanes). ${ }^{1} \mathrm{H} \mathrm{NMR}\left(\delta\right.$, ppm in $\left.\mathrm{CD}_{2} \mathrm{Cl}_{2}\right)$ : 7.16-7.08 (overlapping m, 10H, aromatic $\mathrm{C}-\mathrm{H}$ ), 1.58 (s, $\left.18 \mathrm{H},{ }^{t} \mathrm{Bu}\right) . \mathrm{IR}\left(\mathrm{CH}_{2} \mathrm{Cl}_{2}, \mathrm{~cm}^{-1}\right)$ : 2214 (vs, $\mathrm{CN}_{\mathrm{sym}}$ ), 2188 (vs, $\mathrm{CN}_{\text {asym }}$ ). UV-vis absorption spectrum $\left[\mathrm{CH}_{2} \mathrm{Cl}_{2}, \lambda_{\max }, \mathrm{nm}\left(\varepsilon_{\mathrm{M}}, \mathrm{M}^{-1} \mathrm{~cm}^{-1}\right)\right]$ : 336 (10290). Cyclic 
voltammetry: $6 \mathrm{~d}-\mathrm{e}^{-} \rightarrow[6 \mathrm{~d}]^{1+}$, $+0.66 \mathrm{~V}$. Anal. Calcd for $\mathrm{C}_{24} \mathrm{H}_{28} \mathrm{~N}_{2} \mathrm{~S}_{2} \mathrm{Pt}$ : C, 47.75; H, 4.67; N, 4.64. Found: C, 48.09; H, 4.77; N, 4.78.

[( $\left.\left.\mathbf{P h}_{2} \mathbf{C}_{2} \mathbf{S}_{2}\right) \mathbf{P t}(\mathbf{C N}-\mathbf{1}-\mathbf{A d})_{2}\right], 6 e$. To a solution of $\mathbf{3}(0.150 \mathrm{~g}, 0.221 \mathrm{mmol})$ in $\mathrm{CH}_{2} \mathrm{Cl}_{2}(25 \mathrm{~mL})$ was added $0.716 \mathrm{~g}$ (0.441 mmol) of 1-adamantylisonitrile. Subsequent handling and purification procedures were similar to those implemented for compound $\mathbf{4 b}$, ultimately yielding yellow crystals of $\mathbf{6 e}$. Yield: $0.087 \mathrm{~g}$, $52 \% . \mathrm{R}_{\mathrm{f}}=0.60$ (2:1 $\mathrm{CH}_{2} \mathrm{Cl}_{2}$ :hexanes). ${ }^{1} \mathrm{H}$ NMR $\left(\delta, \mathrm{ppm}\right.$ in $\left.\mathrm{CD}_{2} \mathrm{Cl}_{2}\right): 7.18-7.14(\mathrm{~m}, 4 \mathrm{H}$, aromatic $\mathrm{C}-H)$, 7.12-7.08 (m, 6H, aromatic C-H), 2.36 (m, 18H, Ad), 1.72 (m, 12H, Ad). IR $\left(\mathrm{CH}_{2} \mathrm{Cl}_{2}, \mathrm{~cm}^{-1}\right)$ : 2212 (vs, $\mathrm{CN}_{\text {sym }}$ ), 2192 (vs, $\mathrm{CN}_{\text {asym }}$ ). UV-vis absorption spectrum $\left[\mathrm{CH}_{2} \mathrm{Cl}_{2}, \lambda_{\max }, \mathrm{nm}\left(\varepsilon_{\mathrm{M}}, \mathrm{M}^{-1} \mathrm{~cm}^{-1}\right)\right]$ : 336 (6850). Calcd for $\mathrm{C}_{36} \mathrm{H}_{40} \mathrm{~N}_{2} \mathrm{~S}_{2} \mathrm{Pt}: \mathrm{m} / \mathrm{z}$ 759.943. Observed: $\mathrm{m} / \mathrm{z}$ 760.343. Cyclic voltammetry: $6 \mathbf{e}-\mathrm{e}^{-} \rightarrow[6 \mathbf{e}]^{1+},+0.65 \mathrm{~V}$. Anal. Calcd for $\mathrm{C}_{36} \mathrm{H}_{40} \mathrm{~N}_{2} \mathrm{~S}_{2} \mathrm{Pt}$ : C, 56.90; H, 5.31; N, 3.69. Found: C, 52.53; H, 4.71; N, 3.28.

$\left[\left(\mathbf{P h}_{2} \mathbf{C}_{2} \mathbf{S}_{2}\right) \mathbf{P t}\left(\mathbf{C N}^{t} \mathbf{B u}\right)_{2}\right]\left[\mathbf{S b C l}_{6}\right],[\mathbf{6 d}]\left[\mathbf{S b C l}_{6}\right]$. The following procedure was conducted under an atmosphere of $\mathrm{Ar}$ in oven-dried glassware. A solution of $\mathbf{6 d}(0.119 \mathrm{~g}, 0.198 \mathrm{mmol})$ in dry $\mathrm{CH}_{2} \mathrm{Cl}_{2}(10 \mathrm{~mL})$ cooled to $-20^{\circ} \mathrm{C}$ was dropwise treated via cannula with a solution of tris(4-bromophenyl)ammoniumyl hexachloroantimonate ( $0.161 \mathrm{~g}, 0.197 \mathrm{mmol})$ in $\mathrm{CH}_{2} \mathrm{Cl}_{2}(10 \mathrm{~mL})$ that was similarly chilled to $-20^{\circ} \mathrm{C}$ and protected from ambient light with a wrapping of $\mathrm{Al}$ foil. A dark green color was immediately induced in the mixture. This reaction mixture was stirred at $-20^{\circ} \mathrm{C}$ for $1 \mathrm{~h}$ in the dark, at which point the solvent was removed in vacuo. The residual dark solid was washed with $\mathrm{Et}_{2} \mathrm{O}(3 \mathrm{x} 5 \mathrm{~mL})$ and dried under vacuum. Crystallization of $\left[\left[\left(\mathrm{Ph}_{2} \mathrm{C}_{2} \mathrm{~S}_{2}\right) \mathrm{Pt}\left(\mathrm{CN}^{t} \mathrm{Bu}\right)_{2}\right]_{2}\right]\left[\mathrm{SbCl}_{6}\right]_{2}$ as irregularly-shaped black crystals was accomplished by diffusion of $\mathrm{Et}_{2} \mathrm{O}$ vapor into a $\mathrm{CH}_{2} \mathrm{Cl}_{2}$ solution held at $-20^{\circ} \mathrm{C}$ under $\mathrm{N}_{2}$. This salt is unstable in air, and in solution at room temperature it readily decomposes to $\left[\left(\mathrm{Ph}_{2} \mathrm{C}_{2} \mathrm{~S}_{2}\right)_{2} \mathrm{Pt}\right]$ within minutes of exposure to air. Yield: $0.064 \mathrm{~g}, 17.3 \%$. The following solution spectroscopic data are pertinent a $2[\mathbf{6 d}]^{+} \leftrightarrow[\mathbf{6 d}]_{2}{ }^{2+}$ equilibrium (vide infra) in which the former is believed to dominate: ${ }^{1} \mathrm{H}$ NMR $\left(\delta\right.$, ppm in $\mathrm{CD}_{2} \mathrm{Cl}_{2}$ ): 7.42-7.34 (overlapping m, 10H, aromatic $\mathrm{C}-\mathrm{H}$ ), 1.66, (s, 9H, ${ }^{t} \mathrm{Bu}$ ), 1.59 (s, 9H, $\left.{ }^{t} \mathrm{Bu}\right)$. IR $\left(\mathrm{CH}_{2} \mathrm{Cl}_{2}, \mathrm{~cm}^{-1}\right.$ ): 2243 (vs, $\mathrm{CN}_{\text {sym }}$ ), 2222 (vs, $\mathrm{CN}_{\text {asym }}$ ). UV-vis absorption spectrum $\left[\mathrm{CH}_{2} \mathrm{Cl}_{2}, \lambda_{\max }, \mathrm{nm}\left(\varepsilon_{\mathrm{M}}, \mathrm{M}^{-1} \cdot \mathrm{cm}^{-1}\right)\right]: ~ 439$ (910), 661 (1030), 799 (4050).

$\left[\left(\left(\mathrm{Ph}_{2} \mathrm{C}_{2} \mathrm{~S}_{2}\right) \mathbf{N i}\left(\mathrm{CN}^{t} \mathrm{Bu}\right)_{2}\right)_{2}(\boldsymbol{\mu}-\mathbf{A g})_{2}\right]\left[\mathrm{BF}_{4}\right]_{2},[7]\left[\mathrm{BF}_{4}\right]_{2}$. Under an atmosphere of Ar, a solution of AgBF (0.029 g, $0.15 \mathrm{mmol})$ in $\mathrm{CH}_{2} \mathrm{Cl}_{2}(10 \mathrm{~mL})$ was transferred via cannula to a solution of $\left[\left(\mathrm{Ph}_{2} \mathrm{C}_{2} \mathrm{~S}_{2}\right) \mathrm{Ni}\left(\mathrm{CN}{ }^{t} \mathrm{Bu}\right)_{2}\right]$ $(0.070 \mathrm{~g}, 0.15 \mathrm{mmol})$ in dry $\mathrm{CH}_{2} \mathrm{Cl}_{2}(10 \mathrm{~mL})$. The resulting mixture was stirred overnight in the dark at 25 ${ }^{\circ} \mathrm{C}$ under Ar. This magenta-colored solution was taken to dryness under reduced pressure, and the residual solid was washed with $\mathrm{Et}_{2} \mathrm{O}$ (3 x $10 \mathrm{~mL}$ ). Upon drying, a reddish-magenta solid product was collected. Diffraction quality crystals were obtained by slow diffusion of $\mathrm{Et}_{2} \mathrm{O}$ vapor into a concentrated $\mathrm{CH}_{2} \mathrm{Cl}_{2}$ solution. Yield: 0.079 g, 80\%. ${ }^{1} \mathrm{H}$ NMR ( $\delta$, ppm in $\mathrm{CD}_{2} \mathrm{Cl}_{2}$ ): 7.26-7.18 (m, 20H, aromatic $\left.\mathrm{C}-\mathrm{H}\right), 1.45$ (s, $\left.36 \mathrm{H},{ }^{t} \mathrm{Bu}\right)$. IR $\left(\mathrm{CH}_{2} \mathrm{Cl}_{2}, \mathrm{~cm}^{-1}\right)$ : 2211 (vs, $\mathrm{CN}_{\text {sym }}$ ), 2198 (vs, $\mathrm{CN}_{\text {asym }}$ ). UV-vis absorption spectrum $\mathrm{CCH}_{2} \mathrm{Cl}_{2}$; $\left.\lambda_{\max }, \mathrm{nm}\left(\varepsilon_{\mathrm{M}}, \mathrm{M}^{-1} \cdot \mathrm{cm}^{-1}\right)\right]: 523(\sim 35)$. 
[(2,6-Me 2 py)Ni $\left.\left(\boldsymbol{\mu}_{2}-\boldsymbol{\eta}^{1}, \boldsymbol{\eta}^{1}-\mathrm{S}^{\prime}, \boldsymbol{\eta}^{1}-\mathrm{S}^{\prime \prime}-\mathrm{C}_{2} \mathbf{P h} \mathbf{h}_{2}\right)\right]_{2}, \mathbf{8}$. Via gas-tight syringe, 2,6-dimethylpyridine (0.500 mL, $4.32 \mathrm{mmol})$ was delivered dropwise to a solution of $4 \mathbf{a}(0.050 \mathrm{~g}, 0.130 \mathrm{mmol})$ in toluene $(25 \mathrm{~mL})$ under an argon atmosphere. The mixture was refluxed for $2 \mathrm{~h}$, after which time it was cooled to room temperature. The volume of the solution was reduced by $\sim 2 \mathrm{~mL}$ under vacuum to aid in the removal of displaced MeNC ligand. Reflux in toluene was continued overnight, after which time a minimal quantity of silica gel was added to the flask. The dark green-brown slurry was then taken to dryness in vacuo. This dry-loaded silica was added to the top of a column packed as a silica slurry in hexanes. Elution with $1: 1 \mathrm{CH}_{2} \mathrm{Cl}_{2}$ :hexanes moved a green band of $\left[\left(\mathrm{Ph}_{2} \mathrm{C}_{2} \mathrm{~S}_{2}\right)_{2} \mathrm{Ni}\right]$ followed by brownish-orange 8. Following collection of $\left[\left(\mathrm{Ph}_{2} \mathrm{C}_{2} \mathrm{~S}_{2}\right)_{2} \mathrm{Ni}\right]$, the latter band was fully drawn from the column using 2:1 $\mathrm{CH}_{2} \mathrm{Cl}_{2}$ :hexanes. This fraction was reduced to dryness under reduced pressure, and the residual solid was washed with $n$-pentane and dried further. Recrystallization was achieved by the diffusion of hexanes vapor into a 1,2-dichloroethane solution. Yield: $0.023 \mathrm{~g}, 22 \%$. $\mathrm{R}_{\mathrm{f}}=0.168\left(2: 1 \mathrm{CH}_{2} \mathrm{Cl}_{2}\right.$ :hexanes). ${ }^{1} \mathrm{H} \mathrm{NMR}\left(\delta, \mathrm{ppm}\right.$ in $\left.\mathrm{CD}_{2} \mathrm{Cl}_{2}\right):$ 7.16-7.10 (m, $12 \mathrm{H}$, aromatic $\mathrm{C}-\mathrm{H}), 6.89-6.87(\mathrm{~m}, \sim 2 \mathrm{H}$, aromatic $\mathrm{C}-\mathrm{H}), 6.75-6.66(\mathrm{~m}, \sim 8 \mathrm{H}$, aromatic $\mathrm{C}-\mathrm{H}), 6.56-6.53(\mathrm{~m}, \sim 2 \mathrm{H}$, aromatic $\mathrm{C}-\mathrm{H}), 6.43-6.40(\mathrm{~m}, \sim 2 \mathrm{H}$, aromatic $\mathrm{C}-\mathrm{H}), 3.96\left(\mathrm{~s}, 6 \mathrm{H},-\mathrm{CH}_{3}\right), 3.70\left(\mathrm{~s}, 6 \mathrm{H},-\mathrm{CH}_{3}\right)$. UV-vis absorption spectrum $\left[\mathrm{CH}_{2} \mathrm{Cl}_{2} ; \lambda_{\max }, \mathrm{nm}\left(\varepsilon_{\mathrm{M}}, \mathrm{M}^{-1} \cdot \mathrm{cm}^{-1}\right)\right]: 272$ (8670), 364 (2800), 482 (2230), 693 (260). Cyclic voltammetry (CV): $27+\mathrm{e}^{-} \rightarrow[27]^{1-},+0.05 \mathrm{~V}$; [27] $-\mathrm{e}^{-} \rightarrow[27]^{1+},+0.67 \mathrm{~V}$. Anal. Calcd for $\mathrm{C}_{42} \mathrm{H}_{38} \mathrm{~N}_{2} \mathrm{~S}_{4} \mathrm{Ni}_{2}$ : C, 61.79; H, 4.69; N, 3.43. Found: C, 61.20; H, 4.62; N, 3.45.

[(Ph $\left.\left.\mathbf{C}_{2} \mathbf{S}_{2}\right) \mathbf{N i}(\mathbf{C N M e})\left(\mathbf{P M e}_{3}\right)\right]$, 9. Trimethylphosphine $(0.50 \mathrm{~mL}, 5.0 \mathrm{mmol})$ was delivered dropwise via a gas-tight syringe to a solution of $4 \mathbf{a}(0.039 \mathrm{~g}, 0.100 \mathrm{mmol})$ in $\mathrm{CH}_{2} \mathrm{Cl}_{2}(20 \mathrm{~mL})$, which immediately induce formation of a purple color. The resulting mixture was stirred overnight at $25^{\circ} \mathrm{C}$, after which time the volume of solvent was reduced to $\sim 5 \mathrm{~mL}$ in vacuo. Addition of $\sim 10 \mathrm{~mL} \mathrm{Et}_{2} \mathrm{O}$ led to the precipitation of a purple solid. The solvent was removed by cannula filtration, and the solid was washed with $\mathrm{Et}_{2} \mathrm{O}$ ( 3 x 5 $\mathrm{mL}$ ) to remove unreacted phosphine. The product was dried in vacuo for $1 \mathrm{~h}$ and redissolved in a minimal volume of 1,2-dichloroethane. Filtration of this concentrated solution through Celite and slow introduction of hexanes via vapor diffusion under an inert atmosphere afforded purple crystals. Yield: 0.0227 g, 53\%. $\mathrm{R}_{\mathrm{f}}=0.13$ (2:1 $\mathrm{CH}_{2} \mathrm{Cl}_{2}$ :hexanes). ${ }^{1} \mathrm{H} \mathrm{NMR}\left(\delta\right.$, ppm in $\mathrm{CD}_{2} \mathrm{Cl}_{2}$ ): 7.21-7.06 (overlapping $\mathrm{m}, 10 \mathrm{H}$, aromatic

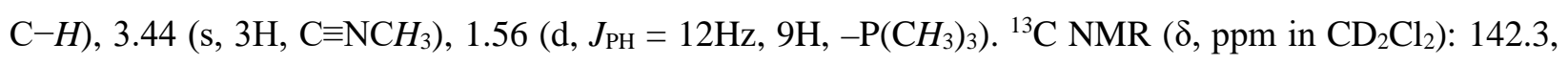
129.70, 129.68, 127.42, 127.36, 125.7, $15.7\left(\mathrm{~d}, J_{\mathrm{PC}}=30 \mathrm{~Hz}, \mathrm{P}\left(\mathrm{CH}_{3}\right)_{3}\right) .{ }^{31} \mathrm{P} \mathrm{NMR}\left(\delta, \mathrm{ppm}\right.$ in $\left.\mathrm{CD}_{2} \mathrm{Cl}_{2}\right): 3.74$. IR $\left(\mathrm{CH}_{2} \mathrm{Cl}_{2}, \mathrm{~cm}^{-1}\right)$ : 2199 (vs, CN). UV-vis absorption spectrum $\left[\mathrm{CH}_{2} \mathrm{Cl}_{2}, \lambda_{\max }, \mathrm{nm}\left(\varepsilon_{\mathrm{M}}, \mathrm{M}^{-1} \cdot \mathrm{cm}^{-1}\right)\right]: 564$ (370).

[( $\left.\left.\mathbf{P h}_{2} \mathbf{C}_{2} \mathbf{S}_{2}\right) \mathbf{N i}(\mathbf{C N M e})\left(\mathbf{P P h}_{3}\right)\right]$, 10. A solid portion of triphenylphosphine (0.113 g, $\left.0.431 \mathrm{mmol}\right)$ was added to a $20 \mathrm{~mL} \mathrm{CH} \mathrm{Cl}_{2}$ solution of $4 \mathbf{a}(0.0413 \mathrm{~g}, 0.108 \mathrm{mmol})$, which caused an immediate color change from blue to green. The resulting mixture was stirred overnight at $25^{\circ} \mathrm{C}$, after which time the solvent was removed under reduced pressure. The residual solid was purified by chromatography on a silica column 
packed as a slurry in hexanes. Unreacted phosphine ligand was separated first by flash elution with 1:1 $\mathrm{CH}_{2} \mathrm{Cl}_{2}$ :hexanes; continued elution with 2:1 $\mathrm{CH}_{2} \mathrm{Cl}_{2}$ :hexanes moved the product as a green band. The solvent was removed under reduced pressure, and the remaining solids were washed with $\mathrm{Et}_{2} \mathrm{O}$. Redissolution in a minimal amount of 1,2-dichloroethane, filtration through Celite, and slow crystallization by the introduction of hexanes via vapor diffusion afforded green crystals of the product. Yield: $0.0177 \mathrm{~g}$, 26\%. $\mathrm{R}_{\mathrm{f}}=0.30$ (2:1 $\mathrm{CH}_{2} \mathrm{Cl}_{2}$ :hexanes). ${ }^{1} \mathrm{H}$ NMR $\left(\delta, \mathrm{ppm}\right.$ in $\left.\mathrm{CD}_{2} \mathrm{Cl}_{2}\right):$ 7.81-7.75 (m, $6 \mathrm{H}$, aromatic $\left.\mathrm{C}-H\right)$, 7.53-7.50 (m, 9H, aromatic $\mathrm{C}-\mathrm{H}), 7.36(\mathrm{~m}, 3 \mathrm{H}$, aromatic $\mathrm{C}-\mathrm{H})$, 7.19-7.06 (overlapping $\mathrm{m}, 10 \mathrm{H}$, aromatic $\mathrm{C}-\mathrm{H}), 2.87$ (s, 3H, C $\left.\equiv \mathrm{NCH}_{3}\right) .{ }^{31} \mathrm{P}$ NMR $\left(\delta\right.$, ppm in $\left.\mathrm{CD}_{2} \mathrm{Cl}_{2}\right)$ : 30.46. IR $\left(\mathrm{CH}_{2} \mathrm{Cl}_{2}, \mathrm{~cm}^{-1}\right): 2204$ (vs, CN). UVvis absorption spectrum $\left[\mathrm{CH}_{2} \mathrm{Cl}_{2}, \lambda_{\max }, \mathrm{nm}\left(\varepsilon_{\mathrm{M}}, \mathrm{M}^{-1} \mathrm{~cm}^{-1}\right)\right]$ : 348 (3900), 463 (210), 622 (190). Calcd for $\mathrm{C}_{34} \mathrm{H}_{28} \mathrm{NPS}_{2} \mathrm{Ni}: \mathrm{m} / \mathrm{z}$ 604.402. Observed: $\mathrm{m} / \mathrm{z} 604.285$.

[(( $\left.\left.\mathbf{P h}_{2} \mathbf{C}_{2} \mathbf{S}_{2}\right) \mathbf{N i}(\mathbf{C} \equiv \mathbf{N M e})\right)_{2}\left(\boldsymbol{\mu}\right.$-dppb)], 11. Under an atmosphere of argon, a $\mathrm{CH}_{2} \mathrm{Cl}_{2}(10 \mathrm{~mL})$ solution of 1,4-dppb (0.312 g, $0.699 \mathrm{mmol})$ in $\mathrm{CH}_{2} \mathrm{Cl}_{2}(10 \mathrm{~mL})$ was added dropwise by cannulation to a solution of $4 \mathbf{a}$ ( $0.135 \mathrm{~g}, 0.352 \mathrm{mmol})$ in $\mathrm{CH}_{2} \mathrm{Cl}_{2}(10 \mathrm{~mL})$. The mixture was stirred at $25{ }^{\circ} \mathrm{C}$ for $\sim 10 \mathrm{~h}$, and progress of the reaction was attended by a change in color from blue to dark green. The solvent was then reduced in volume, and a minimal amount of silica was added to the flask. The resulting slurry was taken to dryness in vacuo. The dry-loaded silica was applied directly to the top of a column packed as a slurry in hexanes. Elution with 1:1 $\mathrm{CH}_{2} \mathrm{Cl}_{2}$ :hexanes moved unreacted ligand from the column; continued elution with 2:1 $\mathrm{CH}_{2} \mathrm{Cl}_{2}$ :hexanes brought forward a well-resolved blue band of [[( $\left.\left.\mathrm{Ph}_{2} \mathrm{C}_{2} \mathrm{~S}_{2}\right)_{2} \mathrm{Ni}\right]$. The desired $\left[\left(\left(\mathrm{Ph}_{2} \mathrm{C}_{2} \mathrm{~S}_{2}\right) \mathrm{Ni}(\mathrm{C} \equiv \mathrm{NMe})\right)_{2}\left(\mu\right.\right.$-dppb)] was flushed from the column as a green band using $5 \% \mathrm{THF}$ in $\mathrm{CH}_{2} \mathrm{Cl}_{2}$. The solvent was removed from the eluant under reduced pressure, and the solid residue was washed with $\sim 15 \mathrm{~mL}$ pentane and dried under vacuum. The product was then dissolved in a minimal volume of $\mathrm{CH}_{2} \mathrm{Cl}_{2}$, filtered through a Celite pad and taken to dryness again. Crystallization was accomplished by slow diffusion of $\mathrm{Et}_{2} \mathrm{O}$ vapor into a concentrated $\mathrm{CH}_{2} \mathrm{Cl}_{2}$ solution of the product. Yield: $0.097 \mathrm{~g}, 49 \%$. $\mathrm{R}_{\mathrm{f}}=0.81(5 \%$ THF: $\left.\mathrm{CH}_{2} \mathrm{Cl}_{2}\right) .{ }^{1} \mathrm{H}$ NMR $\left(\delta\right.$, ppm in $\left.\mathrm{CD}_{2} \mathrm{Cl}_{2}\right)$ : 7.85-7.79 (m, $\sim 6 \mathrm{H}$, aromatic $\left.\mathrm{C}-H\right), 7.74-7.69(\mathrm{~m}, \sim 4 \mathrm{H}$, aromatic C-H), 7.56-7.47 (m, $\sim 10 \mathrm{H}$, aromatic $\mathrm{C}-H$ ), 7.19-7.06 (overlapping $\mathrm{m}, \sim 24 \mathrm{H}$, aromatic $\mathrm{C}-\mathrm{H}$ ), 2.71 (s, 6H, $\left.\mathrm{CNCH}_{3}\right) .{ }^{31} \mathrm{P} \mathrm{NMR}\left(\delta, \mathrm{ppm}\right.$ in $\left.\mathrm{CD}_{2} \mathrm{Cl}_{2}\right): 30.72$. IR $\left(\mathrm{CH}_{2} \mathrm{Cl}_{2}, \mathrm{~cm}^{-1}\right): 2223$ (sh), 2211 (vs, CN). UVvis absorption spectrum $\left[\mathrm{CH}_{2} \mathrm{Cl}_{2} ; \lambda_{\max }, \mathrm{nm}\left(\varepsilon_{\mathrm{M}}, \mathrm{M}^{-1} \cdot \mathrm{cm}^{-1}\right)\right]$ : 468 (520), 618 (420). Cyclic voltammetry: 9 $2 \mathrm{e}^{-} \rightarrow[\mathbf{9}]^{2+},+0.51 \mathrm{~V} ;[\mathbf{9}]^{2+}-2 \mathrm{e}^{-} \rightarrow[\mathbf{9}]^{4+},+1.26 \mathrm{~V}$.

$\left[\left(\left(\mathbf{P h}_{2} \mathrm{C}_{2} \mathrm{~S}_{2}\right) \mathbf{P d}(\mathbf{C N M e})\right)_{2}(\boldsymbol{\mu}\right.$-dppb)], 12. The synthesis and purification procedures were analogous to those used for 11 but with $0.105 \mathrm{~g}(0.244 \mathrm{mmol})$ of $5 \mathrm{a}$ and $0.217 \mathrm{~g}(0.486 \mathrm{mmol})$ of the 1,4-dppb ligand utilized. Crystallization of the product by the diffusion of $\mathrm{Et}_{2} \mathrm{O}$ vapor into a concentrated $\mathrm{CH}_{2} \mathrm{Cl}_{2}$ solution of 12 produced violet diffraction-quality crystals. Yield: $0.121 \mathrm{~g}, 81 \% . \mathrm{R}_{\mathrm{f}}=0.77\left(5 \% \mathrm{THF}: \mathrm{CH}_{2} \mathrm{Cl}_{2}\right) \cdot{ }^{1} \mathrm{H}$ $\operatorname{NMR}\left(\delta\right.$, ppm in $\mathrm{CD}_{2} \mathrm{Cl}_{2}$ ): 7.76-7.68 (overlapping $\mathrm{m}, \sim 12 \mathrm{H}$, aromatic $\mathrm{C}-\mathrm{H}$ ), 7.56-7.49 (overlapping $\mathrm{m}$, $\sim 12 \mathrm{H}$, aromatic $\mathrm{C}-\mathrm{H}), 7.19-7.16(\mathrm{~m}, \sim 4 \mathrm{H}$, aromatic $\mathrm{C}-\mathrm{H}), 7.13-7.05(\mathrm{~m}, \sim 16 \mathrm{H}$, aromatic $\mathrm{C}-\mathrm{H}), 2.87$ (s, 
6H, $\left.\mathrm{CNCH}_{3}\right) .{ }^{31} \mathrm{P}$ NMR ( $\delta$, ppm in $\left.\mathrm{CD}_{2} \mathrm{Cl}_{2}\right):$ 22.62. IR $\left(\mathrm{CH}_{2} \mathrm{Cl}_{2}, \mathrm{~cm}^{-1}\right): \sim 2240$ (sh), 2227 (vs, CN). UV-vis absorption spectrum $\left[\mathrm{CH}_{2} \mathrm{Cl}_{2}, \lambda_{\max }, \mathrm{nm}\left(\varepsilon_{\mathrm{M}}, \mathrm{M}^{-1} \cdot \mathrm{cm}^{-1}\right)\right]: ~ 438$ (890), $\sim 543$ (820). Cyclic voltammetry: 10 $2 \mathrm{e}^{-} \rightarrow[\mathbf{1 0}]^{2+},+0.52 \mathrm{~V} ;[\mathbf{1 0}]^{2+}-2 \mathrm{e}^{-} \rightarrow[\mathbf{1 0}]^{4+},+0.93 \mathrm{~V}$

$\left[\left(\left(\mathbf{P h}_{2} \mathbf{C}_{2} \mathbf{S}_{2}\right) \mathbf{P t}(\mathbf{C N M e})\right)_{2}(\boldsymbol{\mu}\right.$-dppb)], 13. The synthesis and purification procedures were analogous to those employed for $\mathbf{1 1}$ but with $0.246 \mathrm{~g}(0.473 \mathrm{mmol})$ of $\mathbf{6 a}$ and $0.422 \mathrm{~g}(0.945 \mathrm{mmol})$ of the 1,4-dppb ligand utilized. Diffusion of $\mathrm{Et}_{2} \mathrm{O}$ vapor into a concentrated $\mathrm{CH}_{2} \mathrm{Cl}_{2}$ solution of $\mathbf{9}$ produced bright yellow, diffraction-quality crystals. Yield: $0.119 \mathrm{~g}, 36 \% . \mathrm{R}_{\mathrm{f}}=0.75$ (5\% THF: $\left.\mathrm{CH}_{2} \mathrm{Cl}_{2}\right) .{ }^{1} \mathrm{H} \mathrm{NMR}\left(\delta, \mathrm{ppm}\right.$ in $\left.\mathrm{CD}_{2} \mathrm{Cl}_{2}\right)$ : 7.80-7.73 (overlapping $\mathrm{m}, 8 \mathrm{H}$, aromatic $\mathrm{C}-\mathrm{H}$ ), 7.57-7.46 (overlapping $\mathrm{m}, 8 \mathrm{H}$, aromatic $\mathrm{C}-\mathrm{H}$ ), 7.20-7.10 (overlapping $\mathrm{m}, 28 \mathrm{H}$, aromatic $\mathrm{C}-H), 3.48\left(\mathrm{~s}, 6 \mathrm{H}, \mathrm{CNCH}_{3}\right) .{ }^{31} \mathrm{P} \mathrm{NMR}\left(\delta, \mathrm{ppm}\right.$ in $\left.\mathrm{CD}_{2} \mathrm{Cl}_{2}\right): 14.94\left(\mathrm{~s}, J_{\mathrm{Pt}-\mathrm{P}}=\right.$ $2655 \mathrm{~Hz}$ ). IR $\left(\mathrm{CH}_{2} \mathrm{Cl}_{2}, \mathrm{~cm}^{-1}\right)$ : 2237 (sh), 2222 (vs, CN). UV-vis absorption spectrum $\left[\mathrm{CH}_{2} \mathrm{Cl}_{2}, \lambda_{\max }, \mathrm{nm}\right.$ $\left.\left(\varepsilon_{\mathrm{M}}, \mathrm{M}^{-1} \cdot \mathrm{cm}^{-1}\right)\right]: 340$ (11800). Cyclic voltammetry: $11-2 \mathrm{e}^{-} \rightarrow[\mathbf{1 1}]^{2+},+0.57 \mathrm{~V} ;[\mathbf{1 1}]^{2+}-2 \mathrm{e}^{-} \rightarrow[\mathbf{1 1}]^{4+},+1.26$ $\mathrm{V}$.

$\left[\mathbf{E t}_{4} \mathbf{N}\right]_{2}\left[\left(\mathbf{P h}_{2} \mathbf{C}_{2} \mathbf{S}_{2}\right) \mathbf{N i}(\mathbf{C N})_{2}\right],\left[\mathbf{E t}_{4} \mathbf{N}\right]_{2}[\mathbf{1 5}]$. The following is a better-yielding alternative to a preparation reported earlier. ${ }^{10}$ A solution of $\left[\mathrm{Et}_{4} \mathrm{~N}\right]_{2}\left[\mathrm{Ni}(\mathrm{CN})_{4}\right](0.035 \mathrm{~g}, 0.083 \mathrm{mmol})$ in $10 \mathrm{~mL} \mathrm{MeCN}(10 \mathrm{~mL})$ was added to a solution of $\mathbf{4 a}(0.030 \mathrm{~g}, 0.078 \mathrm{mmol})$ in $10 \mathrm{~mL} \mathrm{MeCN}$, which produced a change in color from dark blue to maroon. The solution was stirred overnight under inert atmosphere, after which time the solution volume was reduced to $\sim 3 \mathrm{~mL}$. Addition of $10 \mathrm{~mL} \mathrm{Et}_{2} \mathrm{O}$ induced precipitation of a red solid. The $\mathrm{Et}_{2} \mathrm{O}$ was decanted by use of a cannula, and the solid was dried in vacuo for $1 \mathrm{~h}$. Redissolution in a minimal volume of MeCN, filtration through Celite, and slow crystallization by the introduction of $\mathrm{Et}_{2} \mathrm{O}$ via vapor diffusion afforded red crystals of $\left[\mathrm{Et}_{4} \mathrm{~N}\right]_{2}[15]$. Yield: $0.016 \mathrm{~g}, 0.026 \mathrm{mmol}, 35 \% .{ }^{1} \mathrm{H}$ NMR $(\delta$, ppm in $\left.\mathrm{CD}_{3} \mathrm{CN}\right)$ : 7.16-7.11 (m, 4H, aromatic $\left.\mathrm{C}-\mathrm{H}\right), 7.09-7.02(\mathrm{~m}, 6 \mathrm{H}$, aromatic), 3.36-3.29 (4, 16H, $\left.\mathrm{N}\left(\mathrm{CH}_{2} \mathrm{CH}_{3}\right)_{4}{ }^{+}\right), 1.30\left(\mathrm{t}, J=\sim 5 \mathrm{H}, 24 \mathrm{H}, \mathrm{N}\left(\mathrm{CH}_{2} \mathrm{CH}_{3}\right)_{4}{ }^{+}\right) .{ }^{13} \mathrm{C} \mathrm{NMR}\left(\delta, \mathrm{ppm}\right.$ in $\left.\mathrm{CD}_{3} \mathrm{CN}\right): 145.0,138.0,131.1$, 130.6, 128.9, 126.8, $53.8\left(-\mathrm{CH}_{2}-, \mathrm{Et}_{4} \mathrm{~N}^{+}\right)$, $8.4\left(-\mathrm{CH}_{3}, \mathrm{Et}_{4} \mathrm{~N}^{+}\right)$. IR $\left(\mathrm{CH}_{3} \mathrm{CN}, \mathrm{cm}^{-1}\right): 2111$ (vs, $\left.\mathrm{CN}_{\text {sym }}\right), 2099$ (vs, $\mathrm{CN}_{\text {asym }}$ ). UV-vis absorption spectrum $\left[\mathrm{CH}_{2} \mathrm{Cl}_{2}, \lambda_{\max }, \mathrm{nm}\left(\varepsilon_{\mathrm{M}}, \mathrm{M}^{-1} \cdot \mathrm{cm}^{-1}\right)\right]$ : 489 (220), 378 (5380). Cyclic voltammetry: [15 $]^{2-}-\mathrm{e}^{-} \rightarrow[15]^{1-},-0.07 \mathrm{~V}$.

[Et $\left.{ }_{4} \mathbf{N}\right]\left[\left(\mathbf{P h}_{2} \mathbf{C}_{2} \mathbf{S}_{2}\right) \mathbf{P d}(\mathbf{C N})(\mathbf{C N M e})\right],\left[\mathbf{E t}_{\mathbf{4}} \mathbf{N}\right][\mathbf{1 6}]$. Under an atmosphere of Ar, [Et $\left.{ }_{4} \mathrm{~N}\right][\mathrm{CN}](0.100 \mathrm{~g}, 0.64$ mmol) was added to a solution of $5 \mathbf{a}(0.138 \mathrm{~g}, 0.32 \mathrm{mmol})$ in $\mathrm{CH}_{3} \mathrm{NO}_{2}(25 \mathrm{~mL})$ and the mixture was refluxed overnight with stirring. A gradual color change from red to dark orange was observed as the reaction progressed. After being cooled, the solution was then reduced in volume, and a minimal amount of neutral alumina ( $\sim 1.0 \mathrm{~g}$ ) was added to the flask. The resulting slurry was taken to dryness in vacuo. The dry-loaded alumina was applied directly to the top of a neutral alumina column packed as a slurry in 1:1 $\mathrm{CH}_{2} \mathrm{Cl}_{2}: \mathrm{CH}_{3} \mathrm{CN}$. The column was then eluted with this same solvent mixture. A red band of unreacted started material was collected followed by a well resolved dark orange band of [ $\left.\mathrm{Et}_{4} \mathrm{~N}\right][\mathbf{1 6}]$, which was fully flushed from the column with $100 \% \mathrm{CH}_{3} \mathrm{CN}$. The solvent was removed from the eluant in vacuo, and the 
residue was washed with $\mathrm{Et}_{2} \mathrm{O}(3 \times 10 \mathrm{~mL})$. The residual solid was then dissolved in a minimal volume of $\mathrm{CH}_{3} \mathrm{CN}$, and the resulting solution was filtered through a Celite pad and taken to dryness again. Crystallization of [ $\left.\mathrm{Et}_{4} \mathrm{~N}\right][\mathbf{1 6}]$ was accomplished by slow diffusion of $\mathrm{Et}_{2} \mathrm{O}$ vapor into a concentrated $\mathrm{CH}_{3} \mathrm{CN}$ solution. Yield: $0.104 \mathrm{~g}, 59 \%$. $\mathrm{R}_{\mathrm{f}}=0.69\left(1: 1 \mathrm{CH}_{2} \mathrm{Cl}_{2}: \mathrm{CH}_{3} \mathrm{CN}\right) .{ }^{1} \mathrm{H}$ NMR $\left(\delta\right.$, ppm in $\left.\mathrm{CD}_{3} \mathrm{CN}\right): 7.11-7.01(\mathrm{~m}$, $10 \mathrm{H}$, aromatic $\mathrm{C}-\mathrm{H}), 3.41$ (s, 3H, $\left.\mathrm{CNCH}_{3}\right), 3.16\left(\mathrm{q}, 8 \mathrm{H}, \mathrm{N}\left(\mathrm{CH}_{2} \mathrm{CH}_{3}\right)_{4}{ }^{+}\right), 1.19\left(\mathrm{t}, 12 \mathrm{H}, \mathrm{N}\left(\mathrm{CH}_{2} \mathrm{CH}_{3}\right)_{4}{ }^{+}\right) . \mathrm{IR}$ $\left(\mathrm{CH}_{2} \mathrm{Cl}_{2}, \mathrm{Cm}^{-1}\right)$ : 2227 (vs, $\left.\mathrm{C} \equiv \mathrm{NMe}\right), 2120\left(\mathrm{~s},{ }^{-} \mathrm{C} \equiv \mathrm{N}\right)$. UV-vis absorption spectrum $\left[\mathrm{CH}_{2} \mathrm{Cl}_{2} ; \lambda_{\max }, \mathrm{nm}\left(\varepsilon_{\mathrm{M}}, \mathrm{M}^{-}\right.\right.$ $\left.{ }^{1} \cdot \mathrm{cm}^{-1}\right)$ ]: 338 (9800), 535 (920). Cyclic voltammetry: [16 $]^{1-}-\mathrm{e}^{-} \rightarrow[\mathbf{1 6}],+0.27 \mathrm{~V}$.

$\left[\mathrm{Et}_{4} \mathbf{N}\right]\left[\left(\mathrm{Ph}_{2} \mathrm{C}_{2} \mathrm{~S}_{2}\right) \mathbf{P t}(\mathrm{CN})(\mathrm{CNMe})\right],\left[\mathrm{Et}_{4} \mathbf{N}\right][17]$. The same synthesis and purification procedures used in the synthesis of $\left[\mathrm{Et}_{4} \mathrm{~N}\right][\mathbf{1 6}]$ were employed but with $0.060 \mathrm{~g}(0.38 \mathrm{mmol})$ of $\left[\mathrm{Et}_{4} \mathrm{~N}\right][\mathrm{CN}]$ and $0.066 \mathrm{~g}$ $(0.13 \mathrm{mmol})$ of $\mathbf{6 a}$. Diffraction quality crystals were obtained by slow diffusion of $\mathrm{Et}_{2} \mathrm{O}$ vapor into a saturated $\mathrm{CH}_{3} \mathrm{CN}$ solution of the reddish-orange product. Yield: $0.040 \mathrm{~g}, 49 \%$. $\mathrm{R}_{\mathrm{f}}=0.74(1: 1$ $\mathrm{CH}_{2} \mathrm{Cl}_{2}: \mathrm{CH}_{3} \mathrm{CN}$ ). ${ }^{1} \mathrm{H}$ NMR $\left(\delta\right.$, ppm in $\left.\mathrm{CD}_{2} \mathrm{Cl}_{2}\right):$ 7.17-7.11 (m, 4H, aromatic $\left.\mathrm{C}-\mathrm{H}\right), 7.10-7.03(\mathrm{~m}, 6 \mathrm{H}$, aromatic $\mathrm{C}-\mathrm{H}$ ), 3.38 (s, 3H, $\left.\mathrm{CNCH}_{3}\right), 3.24$ (q, 8H, N( $\left.\left.\mathrm{CH}_{2} \mathrm{CH}_{3}\right)_{4}{ }^{+}\right), 1.26\left(\mathrm{t}, 12 \mathrm{H}, \mathrm{N}\left(\mathrm{CH}_{2} \mathrm{CH}_{3}\right)_{4}{ }^{+}\right) .{ }^{13} \mathrm{C} \mathrm{NMR}$

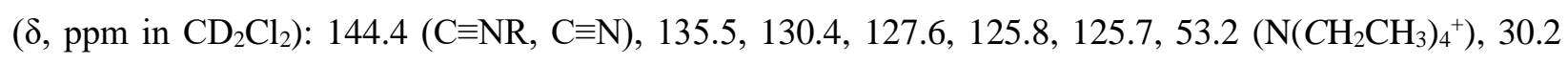
$\left(\mathrm{CNCH}_{3}\right), 7.9\left(\mathrm{~N}\left(\mathrm{CH}_{2} \mathrm{CH}_{3}\right)_{4}{ }^{+}\right)$. IR $\left(\mathrm{CH}_{2} \mathrm{Cl}_{2}, \mathrm{~cm}^{-1}\right)$ : 2219 (vs, $\left.\mathrm{C} \equiv \mathrm{NMe}\right), 2120$ (s, $\left.{ }^{-} \mathrm{C} \equiv \mathrm{N}\right)$. UV-vis absorption spectrum $\left[\mathrm{CH}_{2} \mathrm{Cl}_{2}, \lambda_{\max }, \mathrm{nm}\left(\varepsilon_{\mathrm{M}}, \mathrm{M}^{-1} \mathrm{~cm}^{-1}\right)\right]: 497$ (1350). Cyclic voltammetry: [17] ${ }^{1-}-\mathrm{e}^{-} \rightarrow[17],+0.26 \mathrm{~V}$.

[(Ph $\left.\left.\mathbf{P h}_{2} \mathrm{~S}_{2}\right) \mathbf{N i}(\mathrm{IPr})(\mathbf{C N M e})\right]$, 18. The synthesis, purification and characterization of $\mathbf{1 8}$ have been described previously. ${ }^{10}$

[(Ph $\left.\left.\mathbf{P h}_{2} \mathrm{~S}_{2}\right) \mathbf{P d}(\mathrm{IPr})(\mathrm{CNMe})\right]$, 19. The synthesis and purification procedures were analogous to those used for 18 but with $0.050 \mathrm{~g}(0.116 \mathrm{mmol})$ of 5 a and $0.090 \mathrm{~g}(0.232 \mathrm{mmol})$ of $\mathrm{IPr}^{10}$ Purification was accomplished by flash column chromatography from a silica column eluted with 2:1 $\mathrm{CH}_{2} \mathrm{Cl}_{2}$ :hexanes followed by $100 \% \mathrm{CH}_{2} \mathrm{Cl}_{2}$ to collect $\mathbf{1 9}$ as an orange fraction. Crystallization of $\mathbf{1 9}$ as orange needle crystals was accomplished by the diffusion of hexanes vapor into a concentrated 1,2-dichloroethane solution. Yield: 0.0465 g, 51\%. $\mathrm{R}_{\mathrm{f}}=0.70$ (5\% THF: $\left.\mathrm{CH}_{2} \mathrm{Cl}_{2}\right) .{ }^{1} \mathrm{H}$ NMR $\left(\delta\right.$, ppm in $\left.\mathrm{CD}_{2} \mathrm{Cl}_{2}\right): 7.58$ (t, $J=6 \mathrm{~Hz}, 2 \mathrm{H}$, aromatic $\mathrm{C}-H$ ), 7.40 (d, $J=6 \mathrm{~Hz}, 4 \mathrm{H}$, aromatic $\mathrm{C}-H$ ), 7.25 (s, 2H, carbene -CH=HC-), 7.06-6.97 (overlapping m, $10 \mathrm{H}$, aromatic $\mathrm{C}-\mathrm{H}$ ), 3.24 (s, 3H, $\mathrm{CNCH}_{3}$ ), 2.96 (septet, $\left.J=6 \mathrm{~Hz}, 4 \mathrm{H}, \mathrm{IPr}-\mathrm{CH}\left(\mathrm{CH}_{3}\right)_{2}\right), 1.40$ (d, $J=6 \mathrm{~Hz}$, $12 \mathrm{H}$, IPr $\left.-\mathrm{CH}\left(\mathrm{CH}_{3}\right)_{2}\right), 1.16$ (d, $J=6 \mathrm{~Hz}, 12 \mathrm{H}$, IPr $\left.-\mathrm{CH}\left(\mathrm{CH}_{3}\right)_{2}\right) .{ }^{13} \mathrm{C} \mathrm{NMR}\left(\delta, \operatorname{ppm}\right.$ in $\left.\mathrm{CD}_{2} \mathrm{Cl}_{2}\right): 177.7,146.3$, 144.4, 135.9, 130.7, 130.5, 130.3, 127.4, 127.3, 125.6, 125.4, 124.6, $29.3\left(\mathrm{CNCH}_{3}\right)$, $26.5\left(\mathrm{IPr},-\mathrm{CH}\left(\mathrm{CH}_{3}\right)_{2}\right)$, 22.9 (IPr, - $\left.\mathrm{CH}\left(\mathrm{CH}_{3}\right)_{2}\right)$. IR $\left(\mathrm{CH}_{2} \mathrm{Cl}_{2}, \mathrm{~cm}^{-1}\right)$ : 2213 (vs, CN). UV-vis absorption spectrum $\left[\mathrm{CH}_{2} \mathrm{Cl}_{2}, \lambda_{\max }\right.$, nm $\left.\left(\varepsilon_{\mathrm{M}}, \mathrm{M}^{-1} \cdot \mathrm{cm}^{-1}\right)\right]: 470$ (370). Cyclic voltammetry: $19-\mathrm{e}^{-} \rightarrow[19]^{1+},+0.33 \mathrm{~V}$.

$\left[\left(\mathbf{P h}_{2} \mathrm{C}_{2} \mathrm{~S}_{2}\right) \mathbf{P t}(\mathbf{C}(\mathbf{N M e})(\mathrm{IPr}))(\mathrm{C} \equiv \mathbf{N M e})\right], 20$. The scale of synthesis and procedure for purification were the same as those detailed for $\mathbf{1 9}$. Crude $\mathbf{2 0}$ was purified by flash column chromatography from a silica column eluted with 2:1 $\mathrm{CH}_{2} \mathrm{Cl}_{2}$ :hexanes followed by 5\% THF: $\mathrm{CH}_{2} \mathrm{Cl}_{2}$ to flush it from the column as a dark yellow band. After removal of the eluting solvents, crystallization of $\mathbf{2 0}$ was accomplished by the diffusion 
of hexanes vapor into a concentrated 1,2-dichloroethane solution. Yield: $0.0166 \mathrm{~g}, 19 \%$. $\mathrm{R}_{\mathrm{f}}=0.75$ (5\% THF: $\left.\mathrm{CH}_{2} \mathrm{Cl}_{2}\right) .{ }^{1} \mathrm{H}$ NMR $\left(\delta\right.$, ppm in $\left.\mathrm{CD}_{2} \mathrm{Cl}_{2}\right): 7.56(\mathrm{t}, J=\sim 8 \mathrm{~Hz}, 2 \mathrm{H}$, aromatic $\mathrm{C}-\mathrm{H}), 7.32$ (d, $J=\sim 8 \mathrm{~Hz}, 4 \mathrm{H}$, aromatic $\mathrm{C}-\mathrm{H}) 7.14$ (s, 2H, carbene $-\mathrm{CH}=\mathrm{HC}-$ ), 7.12-6.99 (overlapping m, 10H, aromatic $\mathrm{C}-\mathrm{H}$ ), 3.24 (s, 3H, $-\mathrm{CH}_{3}$ ), 3.03 (s, 3H, $-\mathrm{CH}_{3}$ ), 2.90 (septet, $\left.J=\sim 6 \mathrm{~Hz}, 4 \mathrm{H}, \mathrm{IPr}-\mathrm{CH}\left(\mathrm{CH}_{3}\right)_{2}\right), 1.26$ (d, $J=6 \mathrm{~Hz}, 12 \mathrm{H}, \mathrm{IPr}-$ $\left.\mathrm{CH}\left(\mathrm{CH}_{3}\right)_{2}\right), 1.14$ (d, $J=6 \mathrm{~Hz}, 12 \mathrm{H}$, IPr -CH( $\left.\left(\mathrm{CH}_{3}\right)_{2}\right)$. IR $\left(\mathrm{CH}_{2} \mathrm{Cl}_{2}, \mathrm{Cm}^{-1}\right)$ : 2213 (vs, CN). UV-vis absorption spectrum $\left[\mathrm{CH}_{2} \mathrm{Cl}_{2}, \lambda_{\max }, \mathrm{nm}\left(\varepsilon_{\mathrm{M}}, \mathrm{M}^{-1} \cdot \mathrm{cm}^{-1}\right)\right]$ : 344 (7530). Cyclic voltammetry: $20-\mathrm{e}^{-} \rightarrow[20]^{1+},+0.28 \mathrm{~V}$.

$\left[\left(\mathbf{P h}_{2} \mathbf{C}_{2} \mathrm{~S}_{2}\right) \mathbf{N i}(\mathbf{C N M e})\left(\mathbf{C}(\mathbf{N H M e})\left(\mathrm{NMe}_{2}\right)\right)\right]$, 21. Under an $\mathrm{Ar}$ atmosphere, tetraisopropyl benzobisimidazolium dibromide $(0.100 \mathrm{~g}, 0.20 \mathrm{mmol})$ was transferred into an oven-dried $100 \mathrm{~mL}$ Schlenk flask followed by $\mathrm{NaH}(0.020 \mathrm{~g}, 0.82 \mathrm{mmol})$ and a catalytic amount of potassium tert-butoxide introduced via the tip of a spatula. The flask was affixed to a Schlenk line, and the mixture of solids was suspended in dry THF (50 mL). The mixture was stirred at $25^{\circ} \mathrm{C}$ for $\sim 14 \mathrm{~h}$, during which time smooth bubbling of $\mathrm{H}_{2}$ gas was observed. Separation of the deprotected carbene ligand from the insoluble materials in the mixture was achieved by filtration through a Schlenk frit padded with 1 inch of Celite. The filtrate was transferred dropwise via cannula into a Schlenk flask containing a solution of $\mathbf{4 a}(0.08 \mathrm{~g}, 0.2 \mathrm{mmol})$ in dry THF (10 $\mathrm{mL}$ ). The resulting mixture was stirred for $8 \mathrm{~h}$ at $25^{\circ} \mathrm{C}$, during which time the color changed from dark blue to magenta and then to red. The solvent was removed in vacuo, and the red residual solid was washed with $\mathrm{Et}_{2} \mathrm{O}$ (3 x $10 \mathrm{~mL}$ ). After being dried under vacuum, 21 was crystallized in diffraction quality form by slow diffusion of $\mathrm{CH}_{3} \mathrm{O}^{t} \mathrm{Bu}$ vapor into a concentrated 1,2-dichloroethane solution. Yield: $0.081 \mathrm{~g}, 90.7 \%$. ${ }^{1} \mathrm{H}$ NMR ( $\delta$, ppm in $\mathrm{CD}_{2} \mathrm{Cl}_{2}$ ): 7.19-7.01 (overlapping m, 10H, aromatic $\mathrm{C}-\mathrm{H}$ ), 5.55 (br, $1 \mathrm{H},-\mathrm{NHCH}_{3}$ carbene), 3.92 (s, 3H, - $\mathrm{CH}_{3}$ ), 3.71 (d, $J=6 \mathrm{~Hz}, 3 \mathrm{H},-\mathrm{NHCH}_{3}$ carbene), 3.35 (s, 3H, - $\mathrm{CH}_{3}$ ), 2.94 (s, 3H, $\left.\mathrm{CH}_{3}\right) .{ }^{13} \mathrm{C}$ NMR $\left(\delta\right.$, ppm in $\mathrm{CD}_{2} \mathrm{Cl}_{2}$ ): 204.9, 143.6, 143.3, 137.9, 136.8, 130.2, 130.0, 127.7, 127.6, 125.6, 47.6, 36.6, 36.5, 30.3. IR $\left(\mathrm{CH}_{2} \mathrm{Cl}_{2}, \mathrm{~cm}^{-1}\right)$ : 2197 (vs, CN). UV-vis absorption spectrum $\left[\mathrm{CH}_{2} \mathrm{Cl}_{2}, \lambda_{\max }, \mathrm{nm}\right.$ $\left.\left(\varepsilon_{\mathrm{M}}, \mathrm{M}^{-1} \cdot \mathrm{cm}^{-1}\right)\right]: 510$ (200). Cyclic voltammetry: $24-\mathrm{e}^{-} \rightarrow[24]^{1+},+0.35 \mathrm{~V}$.

\section{Discussion}

Synthesis and Structures. The formation of $\left[\left(\mathrm{Ph}_{2} \mathrm{C}_{2} \mathrm{~S}_{2}\right) \mathrm{M}(\mathrm{C} \equiv \mathrm{NR})_{2}\right]$ from $\left[\left(\mathrm{Ph}_{2} \mathrm{C}_{2} \mathrm{~S}_{2}\right)_{2} \mathrm{M}\right]$ occurs readily under ambient conditions upon the introduction of 2 eq of $\mathrm{C} \equiv \mathrm{NR}$ (Scheme 3, top). With constant metal $\left(\mathrm{Ni}^{2+}\right)$ and isonitrile ligand $\left(\mathrm{CN}^{t} \mathrm{Bu}\right)$, variation in dithiolene ligand substituent among the set $\mathrm{R}^{\prime}=\mathrm{Me}, \mathrm{Ph}$, p-anisyl has no important effect upon either yield or upon physical properties (Scheme 3, bottom). These products are generally amenable to column chromatography purification and standard methods of crystallization.

In our preceding report, ${ }^{10}$ several members of the set $\mathbf{4 a - 4 f ,} 5 \mathbf{5 a - 5 f}$ and $\mathbf{6 a - 6 f}$ were described structurally (4a, 5d, 6f). In this succeeding work, the structures of the remaining members and of a variant with a different dithiolene ligand (4bd) are joined to the array to crystallographic data. Unit cell, refinement 
Table 1. Selected bond lengths ( $\AA$ ), angles (deg.) for 4a-f, 5a-f, 6a-f. ${ }^{a}$

\begin{tabular}{|c|c|c|c|c|c|c|}
\hline & $4 a$ & $4 b$ & $4 \mathbf{c}^{g}$ & $\mathbf{4 d}^{h}$ & $4 e$ & 4f \\
\hline $\mathrm{Ni}-\mathrm{C}_{\text {ave }}{ }^{b}$ & $1.8551[9]^{f}$ & $1.8454[13]$ & $1.848[2]$ & $1.8498(17)$ & $1.848[2]$ & 1.8507[13] \\
\hline $\mathrm{Ni}-\mathrm{S}_{\text {ave }}$ & $2.1427[3]$ & $2.1404[4]$ & $2.1402[2]$ & $2.1344(5)$ & $2.1346[5]$ & $2.1485[6]$ \\
\hline$\Delta^{c}$ & 0.288 & 0.295 & 0.292 & 0.285 & 0.287 & 0.298 \\
\hline $\mathrm{S}-\mathrm{C}_{\mathrm{ave}}$ & $1.7649[8]$ & $1.7571[12]$ & $1.761[2]$ & $1.7577(16)$ & $1.760[2]$ & $1.7688[12]$ \\
\hline $\mathrm{C}-\mathrm{C}_{\text {chelate }}$ & $1.348[1]$ & $1.354(2)$ & $1.346[3]$ & $1.343(3)$ & $1.351[3]$ & $1.355(2)$ \\
\hline $\mathrm{C} \equiv \mathrm{N}_{\mathrm{ave}}$ & $1.149[1]$ & $1.152[1]$ & $1.149[2]$ & $1.149(2)$ & $1.147[2]$ & $1.162[1]$ \\
\hline $\mathrm{C}-\mathrm{Ni}-\mathrm{C}$ & 89.91[5] & $93.44(7)$ & $90.68[11]$ & 93.83(10) & 89.15[10] & $96.15(8)$ \\
\hline $\mathrm{S}-\mathrm{Ni}-\mathrm{S}$ & $90.478[13]$ & $90.613(18)$ & $91.10[3]$ & $90.32(2)$ & $90.79[3]$ & $90.35(3)$ \\
\hline $\mathrm{S}-\mathrm{Ni}-\mathrm{C}_{c i s, \text { ave }}$ & $90.18[3]$ & 88.68[4] & 89.87[5] & $89.02(5)$ & $90.60[5]$ & $88.41[4]$ \\
\hline $\mathrm{S}-\mathrm{Ni}-\mathrm{C}_{\text {trans, ave }}$ & $173.44[3]$ & $170.71[4]$ & 169.13[5] & 168.83(5) & $171.51[5]$ & $165.84[4]$ \\
\hline$\theta,{ }^{d}$ deg. & $6.5,12.2$ & 12.6 & $\begin{array}{c}\text { 14.7, 19.9 } \\
12.5\end{array}$ & 15.6 & $11.7,13.4$ & 19.2 \\
\hline \multirow[t]{2}{*}{$\delta,{ }^{e} \AA$} & $0.064,0.121$ & 0.127 & $\begin{array}{c}0.147,0.194, \\
0.121\end{array}$ & 0.155 & $0.113,0.132$ & 0.195 \\
\hline & $5 a$ & $5 \mathbf{b}$ & $5 c$ & 5d & $5 e$ & $5 f$ \\
\hline $\mathrm{Pd}-\mathrm{C}_{\mathrm{ave}}{ }^{b}$ & $2.002[1]^{f}$ & $1.994[2]$ & $1.9982[11]$ & $1.9955(13)$ & $1.9945[2]$ & $2.0010[13]$ \\
\hline $\mathrm{Pd}-\mathrm{S}_{\mathrm{ave}}$ & $2.2684[1]$ & $2.2708[4]$ & $2.2650[3]$ & $2.2604(3)$ & $2.2625[5]$ & $2.2641[4]$ \\
\hline$\Delta^{c}$ & 0.266 & 0.277 & 0.267 & 0.265 & 0.268 & 0.263 \\
\hline $\mathrm{S}-\mathrm{C}_{\mathrm{ave}}$ & $1.762[1]$ & $1.761[1]$ & $1.7652[9]$ & $1.7606(12)$ & $1.765[2]$ & $1.7668[11]$ \\
\hline $\mathrm{C}-\mathrm{C}_{\text {chelate }}$ & $1.351(3)$ & $1.352(3)$ & $1.3555(17)$ & $1.351(2)$ & $1.347(3)$ & $1.356(2)$ \\
\hline $\mathrm{C} \equiv \mathrm{N}_{\text {ave }}$ & $1.135[2]$ & $1.145[2]$ & $1.1442[14]$ & $1.1465(17)$ & $1.145[3]$ & $1.146[1]$ \\
\hline $\mathrm{C}-\mathrm{Pd}-\mathrm{C}$ & $91.8[4]^{i}$ & $92.07(1)$ & $88.48(6)$ & 93.33(7) & $96.79(10)$ & $92.20(7)$ \\
\hline $\mathrm{S}-\mathrm{Pd}-\mathrm{S}$ & $88.87(2)$ & $88.940(19)$ & 88.678(12) & $88.028(16)$ & $88.76(2)$ & 88.356(15) \\
\hline $\mathrm{S}-\mathrm{Pd}-\mathrm{C}_{c i s, \text { ave }}$ & $89.6[3]^{i}$ & $89.63[5]$ & $91.44[3]$ & $89.92(4)$ & 87.27[5] & $90.76[4]$ \\
\hline $\mathrm{S}-\mathrm{Pd}-\mathrm{C}_{\text {trans,ave }}$ & $175.7[2]^{i}$ & $175.52[5]$ & $178.64[3]$ & 171.28(4) & $175.27[5]$ & $175.13[4]$ \\
\hline$\theta,{ }^{d}$ deg. & $2.3,8.7^{j}$ & 5.5 & 1.9 & 11.7 & 3.3 & 4.4 \\
\hline \multirow[t]{2}{*}{$\delta,{ }^{e} \AA$} & $0.083,0.024$ & 0.059 & 0.020 & 0.123 & 0.036 & 0.044 \\
\hline & $6 a$ & $6 \mathbf{b}$ & $6 c$ & 6d & $6 e$ & $6 f$ \\
\hline $\mathrm{Pt}-\mathrm{C}_{\mathrm{ave}}{ }^{b}$ & $1.955[2]^{f}$ & $1.973[2]$ & $1.964[3]$ & $1.965[2]$ & $1.957[2]$ & $1.965[3]$ \\
\hline $\mathrm{Pt}-\mathrm{S}_{\mathrm{ave}}$ & $2.2757[5]$ & $2.2829[6]$ & $2.2747[7]$ & $2.2773[5]$ & $2.2745[6]$ & $2.2748[7]$ \\
\hline$\Delta^{c}$ & 0.321 & 0.310 & 0.311 & 0.312 & 0.318 & 0.310 \\
\hline $\mathrm{S}-\mathrm{C}_{\mathrm{ave}}$ & $1.765[2]$ & $1.760[2]$ & $1.762[2]$ & $1.763[2]$ & $1.760[2]$ & $1.764[3]$ \\
\hline $\mathrm{C}-\mathrm{C}_{\text {chelate }}$ & $1.349[4]$ & $1.353(4)$ & $1.353(3)$ & $1.343(4)$ & $1.353(4)$ & $1.345(5)$ \\
\hline $\mathrm{C} \equiv \mathrm{N}_{\mathrm{ave}}$ & $1.146[3]$ & $1.134[3]$ & $1.136[3]$ & $1.145[3]$ & $1.150[3]$ & $1.138[4]$ \\
\hline $\mathrm{C}-\mathrm{Pt}-\mathrm{C}$ & 91.38[11] & $91.43(12)$ & 88.36(16) & $96.13(12)$ & $96.08(13)$ & $90.70(7)$ \\
\hline $\mathrm{S}-\mathrm{Pt}-\mathrm{S}$ & $88.74[2]$ & 89.13(3) & $88.77(3)$ & 89.12(2) & 88.84(3) & $91.512(17)$ \\
\hline $\mathrm{S}-\mathrm{Pt}-\mathrm{C}_{c i s, \text { ave }}$ & 89.97[6] & $89.78[6]$ & $91.45[8]$ & $87.37[6]$ & $87.58[7]$ & 89.99[4] \\
\hline $\mathrm{S}-\mathrm{Pt}-\mathrm{C}_{\text {trans,ave }}$ & $177.67[6]$ & $178.51[6]$ & $178.50[6]$ & $176.27[6]$ & $175.66[7]$ & 176.67[4] \\
\hline$\theta,{ }^{d}$ deg. & $3.7,1.6$ & 4.1 & 2.1 & 1.9 & 3.2 & 4.0 \\
\hline$\delta,{ }^{e} \AA$ & $0.039,0.013$ & 0.044 & 0.021 & 0.008 & 0.034 & 0.041 \\
\hline
\end{tabular}

${ }^{a}$ The structures of $\mathbf{4 a}, \mathbf{5 d}$, and $\mathbf{6 f}$ were reported previously. ${ }^{7}$ Structural data are included here to assist identification of general trends. ${ }^{b}$ Uncertainties are propagated according to Taylor, J. R. An Introduction to Error Analysis; 2nd ed.; University Science Books: Sausalito, CA, 1997, pp 73-77. ${ }^{c} \Delta=$ M-Save $\mathrm{M}-\mathrm{C}_{\text {ave }} ;{ }^{d} \theta=$ angle between $\mathrm{C}-\mathrm{M}-\mathrm{C}$ and $\mathrm{S}-\mathrm{M}-\mathrm{S}$ planes; ${ }^{e}$ Average atom displacement from $\mathrm{MS}_{2} \mathrm{C}_{2}$ mean plane; fSquare brackets designate propagated uncertainty; ${ }^{9}$ Values are from one whole molecule and two half molecules on special positions in the unit cell. ${ }^{h}$ Values are not averaged, as only a half molecule occurs in the asymmetric unit of the cell. ${ }^{i}$ High uncertainties originate from the presence of a disordered MeNC, which was used in averaging. ${ }^{j}$ Two values occur due to a MeNC ligand disordered over two positions. 


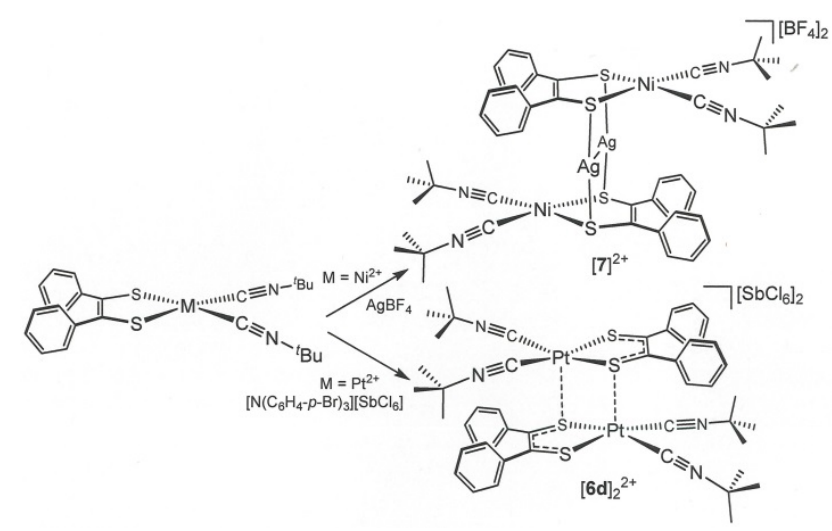

Scheme 4. Outcomes from reaction of $\left[\left(\mathrm{Ph}_{2} \mathrm{C}_{2} \mathrm{~S}_{2}\right) \mathrm{M}\left(\mathrm{CN}^{t} \mathrm{Bu}\right)_{2}\right]$ with oxidizing agents.

indices and other pertinent crystallographic data are available in supporting information (Tables S1-S4), while representative structures are depicted in Figure 1. Selected bond lengths and angles, presented where possible as averaged values, are gathered into Table 1. Considered in the aggregate, these data identify several trends: 1) A tendency toward greater planarity, as gauged by $\theta$, the angle between the $\mathrm{MS}_{2}$ and $\mathrm{MC}_{2}$ planes, and by $\delta$, the average atom displacement $(\AA)$ from the $\mathrm{MS}_{2} \mathrm{C}_{2}$ mean plane, upon descending the Group 10 metals from Ni to Pd to Pt (Table 1). This progression is due to the stronger ligand field enjoyed by the $2^{\text {nd }}$ and $3^{\text {rd }}$ row metals, in a constant ligand environment, as compared to a first row metal. 2) A lower value of $\Delta$, the difference between $\mathrm{M}-\mathrm{S}$ and $\mathrm{M}-\mathrm{C}$ bond lengths, in moving from $\mathrm{Ni}$ to $\mathrm{Pd}$ and then an increase in moving from Pd to Pt. This change arises from either an enhanced Pd-S interaction or diminished $\mathrm{Pd}-\mathrm{C}$ bonding - or both - relative to the Ni and Pt compounds. 3) A modest decrease in the isonitrile $\mathrm{C} \equiv \mathrm{N}$ bond length in moving from $\mathrm{Ni}^{2+}$ to $\mathrm{Pd}^{2+}$ to $\mathrm{Pt}^{2+}$ at parity of ligand such that, in more instances than not, the difference in $\mathrm{C} \equiv \mathrm{N}$ for the nickel compound vs the platinum compound is significant by the $3 \sigma$ criterion (Table 1, bold type). The meaningfulness of this trend is corroborated by $v_{\mathrm{CN}}$ (vide infra), which trend to higher energy as metal varies from $\mathrm{Ni}^{2+}$ to $\mathrm{Pd}^{2+}$ to $\mathrm{Pt}^{2+}$.

Compounds of the type $\left[\left(\mathrm{Ph}_{2} \mathrm{C}_{2} \mathrm{~S}_{2}\right) \mathrm{M}(\mathrm{C} \equiv \mathrm{NR})_{2}\right]$ generally support a single one-electron oxidation at $\sim+0.50 \mathrm{~V}$ vs. AgCl/Ag (vide infra), which is attributed to transformation of the ene-1,2-dithiolate to radical monoanion (Scheme 1). Among redox agents with oxidizing power nominally sufficient to generate cationic $\left[\left(\mathrm{Ph}_{2} \mathrm{C}_{2} \mathrm{~S}_{2}\right) \mathrm{M}(\mathrm{C} \equiv \mathrm{NR})_{2}\right]^{+}$is $\mathrm{Ag}^{+}$in $\mathrm{CH}_{2} \mathrm{Cl}_{2} \cdot{ }^{19}$ Introduction of $\mathrm{AgBF}_{4}$ in $\mathrm{CH}_{2} \mathrm{Cl}_{2}$ to $\left[\left(\mathrm{Ph}_{2} \mathrm{C}_{2} \mathrm{~S}_{2}\right) \mathrm{Ni}\left(\mathrm{C} \equiv \mathrm{N}^{t} \mathrm{Bu}\right)_{2}\right]$ produces dimetallic $\left[\left[\left(\mathrm{Ph}_{2} \mathrm{C}_{2} \mathrm{~S}_{2}\right) \mathrm{Ni}\left(\mathrm{C} \equiv \mathrm{N}^{t} \mathrm{Bu}\right)_{2}\right]_{2}(\mu-\mathrm{Ag})_{2}\right]\left[\mathrm{BF}_{4}\right]_{2}\left([7]\left[\mathrm{BF}_{4}\right]_{2}\right.$, Scheme 4), wherein two closely juxtaposed $\mathrm{Ag}^{+}$ions are flanked by the dithiolene sulfurs atoms of two parallel $\left[\left(\mathrm{Ph}_{2} \mathrm{C}_{2} \mathrm{~S}_{2}\right) \mathrm{Ni}\left(\mathrm{C} \equiv \mathrm{N}^{t} \mathrm{Bu}\right)_{2}\right]$ units such that the midpoint of the $\mathrm{Ag}_{2}{ }^{2+}$ unit coincides with an inversion center and only half of the overall assembly is structurally unique. Intraligand $\mathrm{S}-\mathrm{C}$ and $\mathrm{C}-\mathrm{C}_{\text {chelate }}$ bonds in [7] ${ }^{2+}$ (Table 2, Scheme 1) remain consistent with ene-1,2-dithiolate formulation of the ligand and indicate that electron transfer did not occur. Rather, the $\mathrm{Ag}^{+}$ions have simply been ensconced between the soft thiolate 


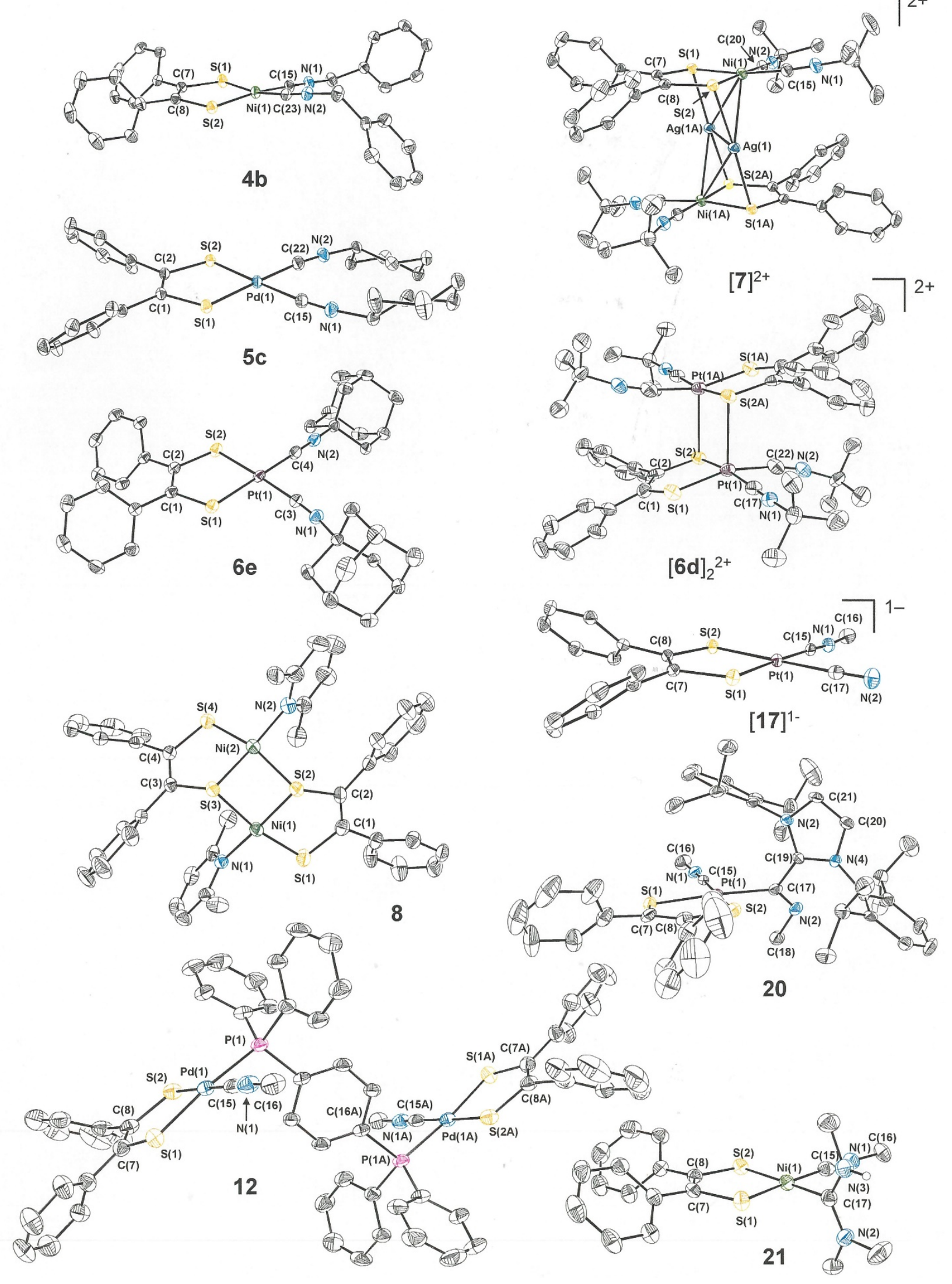

Figure 1. Representative thermal ellipsoid plots at the 50\% probability level of compounds from Schemes 3, 4 and 5. Hydrogen atoms are omitted for clarity. 
Table 2. Selected bond lengths $(\AA)$, angles (deg.) for $[7]^{2+}$ and $[6 \mathbf{6 d}]_{2}^{2+}$.

\begin{tabular}{|c|c|c|c|}
\hline \multicolumn{2}{|c|}{$[7]^{2+}$} & \multicolumn{2}{|c|}{$[\mathbf{6 d}]_{2^{2+}}$} \\
\hline $\mathrm{Ni}-\mathrm{C}_{\text {ave }}$ & $1.863[2]$ & $\mathrm{Pt}-\mathrm{C}^{d}$ & $1.986[7]$ \\
\hline $\mathrm{Ni}-\mathrm{S}_{\text {ave }}$ & $2.1631[4]$ & $\mathrm{Pt}-\mathrm{C}^{e}$ & $1.997[9]$ \\
\hline$\Delta^{a}$ & 0.300 & $\mathrm{Pt}-\mathrm{S}^{f}$ & $2.263[1]$ \\
\hline $\mathrm{S}-\mathrm{C}_{\mathrm{ave}}$ & $1.7626[13]$ & $\mathrm{Pt}-\mathrm{S}^{g}$ & $2.272[1]$ \\
\hline $\mathrm{C}-\mathrm{C}_{\text {chelate }}$ & $1.348(2)$ & $\Delta^{a}$ & 0.276 \\
\hline $\mathrm{C} \equiv \mathrm{N}_{\text {ave }}$ & $1.146[2]$ & $\mathrm{C} \equiv \mathrm{N}_{\mathrm{ave}}$ & $1.131[6]$ \\
\hline S-Agave & $2.4403[4]$ & $\mathrm{Pt} \cdots \cdot \mathrm{S}^{h}$ & $2.749[1]$ \\
\hline $\mathrm{Ni}-\mathrm{Ag}_{\text {ave }}$ & $2.9442[2]$ & $\mathrm{S}-\mathrm{C}^{i}$ & $1.708[7]$ \\
\hline 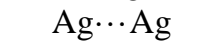 & 3.0921(3) & $\mathrm{S}-\mathrm{C}^{j}$ & $1.726[7]$ \\
\hline $\mathrm{Ni} \cdots \mathrm{Ni}$ & 5.011 & $\mathrm{C}-\mathrm{C}_{\text {chelate }}$ & $1.384[9]$ \\
\hline$\delta,^{b} \AA$ & 0.054 & $\delta,{ }^{b} \AA$ & $0.216,0.223$ \\
\hline $\mathrm{C}-\mathrm{Ni}-\mathrm{C}$ & 92.94(9) & $\mathrm{C}-\mathrm{Pt}-\mathrm{C}$ & $90.2[3]$ \\
\hline $\mathrm{S}-\mathrm{Ni}-\mathrm{S}$ & 90.877(19) & $\mathrm{S}-\mathrm{Pt}-\mathrm{S}^{k}$ & $87.70[6]$ \\
\hline $\mathrm{S}-\mathrm{Ni}-\mathrm{C}_{c i s, \text { ave }}$ & $88.26[5]$ & $\mathrm{S}_{\mathrm{br}}-\mathrm{Pt}-\mathrm{S}_{\mathrm{br}}{ }^{l}$ & $94.58[5]$ \\
\hline $\mathrm{S}-\mathrm{Ni}-\mathrm{C}_{\text {trans,ave }}$ & 173.85[4] & $\mathrm{S}_{\text {non br }}-\mathrm{Pt}-\mathrm{S}_{\mathrm{br}}{ }^{m}$ & 103.42[6] \\
\hline Ag-Ni-Ag & 63.352(7) & $\mathrm{S}-\mathrm{Pt}-\mathrm{C}_{\text {trans, non br }}{ }^{n}$ & $162.4[2]$ \\
\hline $\mathrm{Ni}-\mathrm{Ag}-\mathrm{Ni}$ & 116.648(7) & $\mathrm{S}-\mathrm{Pt}-\mathrm{C}_{\text {trans, br }}{ }^{\circ}$ & $173.1[2]$ \\
\hline$\theta,{ }^{c}$ deg. & 8.7 & $\theta,{ }^{c}$ deg. & $18.3,18.9$ \\
\hline
\end{tabular}

${ }^{a} \Delta=\mathrm{M}-\mathrm{S}_{\text {ave }}-\mathrm{M}-\mathrm{C}_{\text {ave }}$; ${ }^{b}$ Displacement of $\mathrm{M}$ above the $\mathrm{S}_{2} \mathrm{C}_{2}$ mean plane. ${ }^{c} \theta=$ angle between $\mathrm{C}_{2} \mathrm{M}$ and $\mathrm{S}_{2} \mathrm{M}$ planes; ' Isonitrile carbon trans to bridging sulfur; ' Isonitrile carbon cis to bridging sulfur; 'Dithiolene sulfur not involved in bridging; ${ }^{g}$ Dithiolene sulfur involved in bridging; ${ }^{h} \mathrm{Pt} \cdots \mathrm{S}$ interaction through which dimerization occurs; ${ }^{i}$ Dithiolene sulfur not involved in dimer formation; ${ }^{j}$ Dithiolene sulfur involved in dimeric interaction; ${ }^{k}$ Intraligand S-Pt-S angle; ${ }^{l}$ Dithiolene sulfur atoms involved in bridging. ${ }^{m}$ Angle defined by nonbridging sulfur and bridging dithiolene sulfur from other half of dimer; ${ }^{n}$ trans $\mathrm{S}-\mathrm{Pt}-\mathrm{C}$ angle involving dithiolene sulfur not involved in bridging; ${ }^{\circ}$ trans S-Pt-C angle involving dithiolene sulfur involved in bridging.

sulfur atoms. A similar outcome was found with [(dcpe) $\left.\mathrm{Ni}\left(\mathrm{S}_{2} \mathrm{C}_{6} \mathrm{H}_{2} \mathrm{~S}_{2}\right) \mathrm{Ni}(\mathrm{dcpe})\right]$ (dcpe = 1,2bis(dicyclohexylphosphino)ethane; $\left[\mathrm{S}_{2} \mathrm{C}_{6} \mathrm{H}_{2} \mathrm{~S}_{2}\right]^{4-}=1,2,4,5$-tetrathiolatobenzene) in reaction with $\mathrm{Ag}[\mathrm{B}(3,5$ $\left.\left.-\left(\mathrm{CF}_{3}\right)_{2}-\mathrm{C}_{6} \mathrm{H}_{3}\right)_{4}\right],{ }^{20}$ and other complexes featuring the $\mathrm{Ag}_{2}{ }^{2+}$ unit in an environment of sulfur donor atoms have been described..$^{21-22}$

Triarylaminium cations such as $\left[\mathrm{N}\left(\mathrm{C}_{6} \mathrm{H}_{4}-p-\mathrm{Br}\right)_{3}\right]^{+}$are both more potent oxidizing agents and uncomplicated by any tendency to act as ligand. Treatment of a $\mathrm{CH}_{2} \mathrm{Cl}_{2}$ solution of $\left[\left(\mathrm{Ph}_{2} \mathrm{C}_{2} \mathrm{~S}_{2}\right) \mathrm{Pt}\left(\mathrm{C} \equiv \mathrm{N}^{t} \mathrm{Bu}\right)_{2}\right]$ with $\left[\mathrm{N}\left(\mathrm{C}_{6} \mathrm{H}_{4}-p-\mathrm{Br}\right)_{3}\right]\left[\mathrm{SbCl}_{6}\right]$ induces an immediate and pronounced darkening in color. Following removal of $\left[\mathrm{N}\left(\mathrm{C}_{6} \mathrm{H}_{4}-p-\mathrm{Br}\right)_{3}\right]$ upon washing, recrystallization from $\mathrm{CH}_{2} \mathrm{Cl}_{2} / \mathrm{Et}_{2} \mathrm{O}$ provides crystalline $\left[\left[\left(\mathrm{Ph}_{2} \mathrm{C}_{2} \mathrm{~S}_{2}\right) \mathrm{Pt}\left(\mathrm{C} \equiv \mathrm{N}^{t} \mathrm{Bu}\right)_{2}\right]_{2}\right]\left[\mathrm{SbCl}_{6}\right]_{2} \cdot 2 \mathrm{CH}_{2} \mathrm{Cl}_{2} \quad\left([\mathbf{6 d}]_{2}\left[\mathrm{SbCl}_{6}\right]_{2} \cdot 2 \mathrm{CH}_{2} \mathrm{Cl}_{2}\right)$, which was identified by X-ray

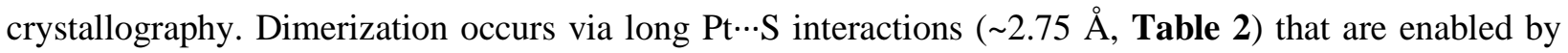
moderate pyramidalization at the $\mathrm{Pt}^{2+}$ ions. This pyramidalization lifts the $\mathrm{Pt}^{2+}$ ions $\sim 0.22 \AA$ above the $\mathrm{S}_{2} \mathrm{C}_{2}$ mean plane and folds the $\mathrm{PtS}_{2}$ and $\mathrm{PtC}_{2}$ planes to a dihedral angle of $\sim 18.5^{\circ}$ from their near planarity in charge-neutral 6d (Table 2). The center of the resulting $\mathrm{Pt}_{2} \mathrm{~S}_{2}$ rhomb resides on an inversion center in triclinic $P-1$. Intraligand $\mathrm{S}-\mathrm{C}$ and $\mathrm{C}-\mathrm{C}_{\text {chelate }}$ bond lengths in $[\mathbf{6 d}]_{2}{ }^{2+}$ are appreciably shorter and longer, respectively, than the corresponding values in $\mathbf{6 d}(c f$. Table 1). These bond length changes indicate 


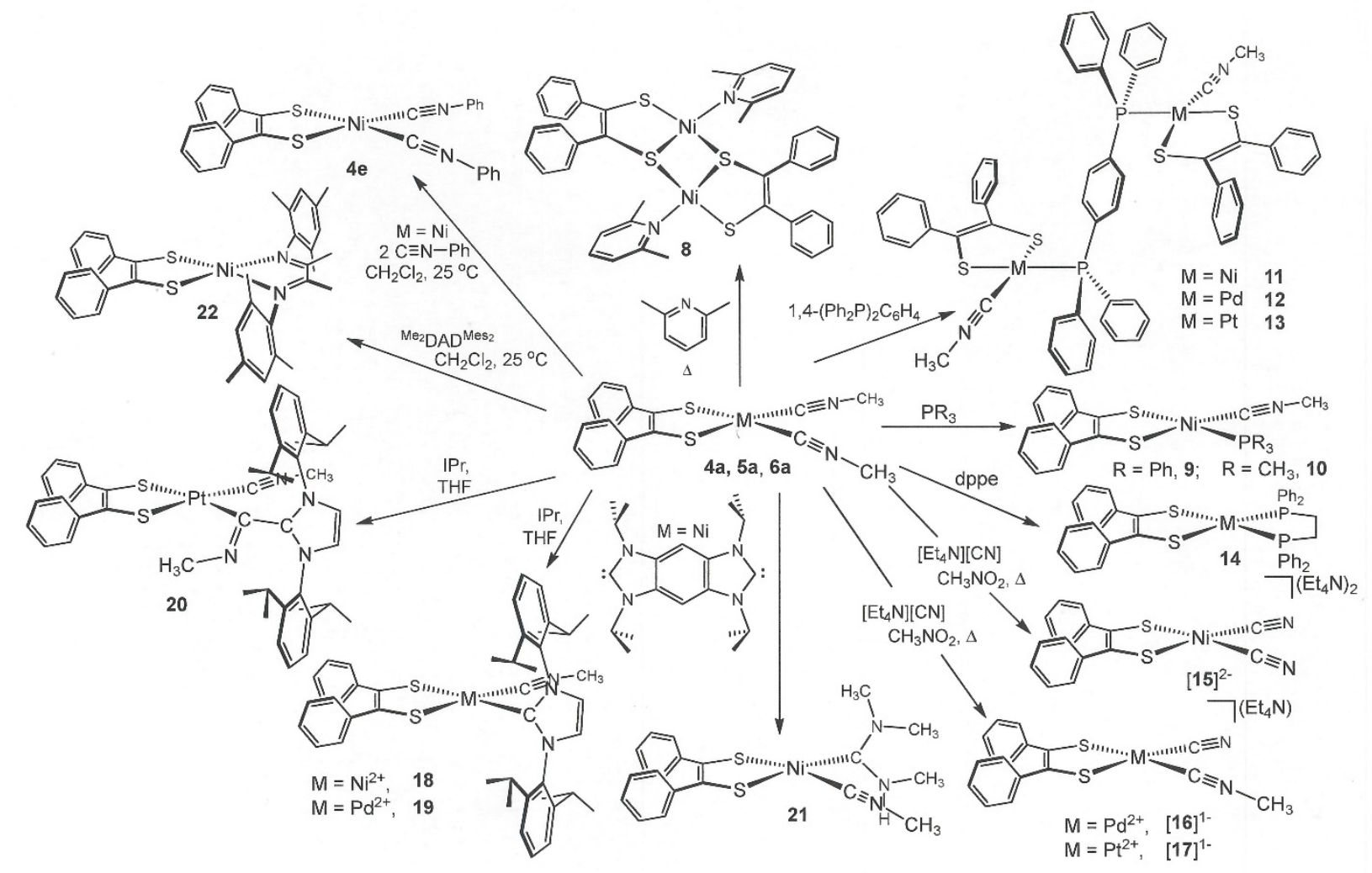

Scheme 5. Substitution reactions of $\left[\left(\mathrm{Ph}_{2} \mathrm{C}_{2} \mathrm{~S}_{2}\right) \mathrm{M}\left(\mathrm{CNCH}_{3}\right)_{2}\right]\left(\mathrm{M}=\mathrm{Ni}^{2+}, \mathrm{Pd}^{2+}, \mathrm{Pt}^{2+}\right)$.

transformation of the dithiolene ligand from ene-1,2-dithiolate to radical monoanion (Scheme 1), which simultaneously introduces multiple bond character between sulfur and carbon and lowers the $\mathrm{C}-\mathrm{C}_{\text {chelate }}$ bond order. The general dimeric structure exemplified in $[\mathbf{6 d}]_{2}{ }^{2+}$ is typical for homoleptic $\left[M\left(S_{2} C_{2} R_{2}\right)_{2}\right]^{0,1-2-}(M$ $=\mathrm{Fe}, \mathrm{Co})$ complexes $^{23-24}$ but is a less common structure type for heteroleptic transition metal dithiolene complexes. A similar dimeric structure held by axial Pt $\cdots$ S interactions has been observed for [[ $\left({ }^{t} \mathrm{Bu}-p-\right.$ $\left.\left.\mathrm{C}_{6} \mathrm{H}_{4}\right)_{2} \mathrm{C}_{2} \mathrm{~S}_{2}\right) \mathrm{Pt}\left(4,4^{\prime}-{ }^{t} \mathrm{Bu}_{2}\right.$-bipy $\left.\left.)\right]_{2}\right]\left[\mathrm{PF}_{6}\right]_{2}{ }^{25}$

Following an initial limited set of observed displacements of MeNC from $\left[\left(\mathrm{Ph}_{2} \mathrm{C}_{2} \mathrm{~S}_{2}\right) \mathrm{Ni}(\mathrm{C} \equiv \mathrm{NMe})_{2}\right],{ }^{10}$ we have more thoroughly probed the breadth of substitution reactions that can be accomplished by new ligands across the $\left[\left(\mathrm{Ph}_{2} \mathrm{C}_{2} \mathrm{~S}_{2}\right) \mathrm{M}(\mathrm{C} \equiv \mathrm{NMe})_{2}\right](\mathrm{M}=\mathrm{Ni}, \mathrm{Pd}, \mathrm{Pt})$ series. Scheme 5 presents the full range of results observed thus far with $\left[\left(\mathrm{Ph}_{2} \mathrm{C}_{2} \mathrm{~S}_{2}\right) \mathrm{M}(\mathrm{C} \equiv \mathrm{NMe})_{2}\right]\left(\mathrm{M}=\mathrm{Ni}^{2+}, \mathrm{Pd}^{2+}, \mathrm{Pt}^{2+}\right)$, including those disclosed previously. Treatment of $\mathbf{4 a}$ with excess 2,6-dimethylpyridine led to modest yields of dimetallic $\mathbf{8}$, which is noteworthy among the compounds of Scheme 5 in being only one of two outcomes showing complete displacement of MeNC by a monodentate ligand. The two $\mathrm{Ni}^{2+}$ ions are bridged by one thiolate sulfur from each dithiolene ligand but in a slightly asymmetric fashion in which the shorter $\mathrm{Ni}-\mathrm{S}$ bond is formed with the sulfur atom from the dithiolene ligand that chelates the metal ion. The difference in $\mathrm{Ni}-\mathrm{S}_{\mathrm{br}}$ bond lengths is $\sim 0.07$ (Table 3). The central $\mathrm{Ni}_{2} \mathrm{~S}_{2}$ core shows a distinctive "butterfly" fold along the $\mathrm{Ni} \cdots \mathrm{Ni}$ axis such that the two Ni- $\mathrm{S}_{b r}-\mathrm{Ni}$ planes meet at a $62.8^{\circ}$ angle (Figure 1). The nearly planar $\mathrm{C}_{2} \mathrm{~S}_{2} \mathrm{Ni}$ metallodithiolene groups at the two ends of the molecule are, in consequence of the folding within the central $\mathrm{Ni}_{2} \mathrm{~S}_{2}$ core, 
Table 3. Selected interatomic distances $(\AA)$ and angles (deg.) for $\mathbf{8}$.

\begin{tabular}{c|c}
\hline & $\mathbf{8}$ \\
\hline $\mathrm{Ni} \cdots \mathrm{Ni}$ & $2.8406(9)$ \\
$\mathrm{Ni}-\mathrm{N}$ & $1.941[3]$ \\
$\mathrm{Ni}-\mathrm{S}_{\text {nonbridging }}$ & $2.1508[10]$ \\
$\mathrm{Ni}-\mathrm{S}_{\text {bridging, cis to N }}$ & $2.2158[10]$ \\
$\mathrm{Ni}-\mathrm{S}_{\text {bridging, trans to N }}$ & $2.1411[9]$ \\
$\mathrm{Ni}-\mathrm{S}_{\mathrm{br}}-\mathrm{Ni}$ & $81.36[3]$ \\
$\mathrm{S}_{\mathrm{br}}-\mathrm{Ni}-\mathrm{S}_{\mathrm{br}}$ & $80.68[4]$ \\
$\mathrm{S}-\mathrm{Ni}-\mathrm{S}_{\text {trans }}$ & $167.33[4]$ \\
$\mathrm{S}-\mathrm{Ni}-\mathrm{S}_{\mathrm{chelate}}$ & $89.72[4]$ \\
$\mathrm{N}-\mathrm{Ni}-\mathrm{S}_{\text {br,trans }}$ & $174.09[9]$ \\
$\varphi^{a}$, deg. & 74.3 \\
$\tau^{b}$, deg. & 62.8 \\
\hline
\end{tabular}

${ }^{a}$ Angle between $\left(\mathrm{C}_{2} \mathrm{~S}_{2}\right) \mathrm{Ni}$ coordination planes; ${ }^{b}$ Fold angle between $\mathrm{Ni}-\mathrm{S}_{\mathrm{br}}-\mathrm{Ni}$ planes.

disposed at an angle of $74.3^{\circ}$. Similar structural features have been observed in dipalladium dithiolene complexes bearing $\mathrm{Ph}_{3} \mathrm{P}$ in place of 2,6-dimethylpyridine. ${ }^{26-27}$

With either $\mathrm{PPh}_{3}$ or $\mathrm{PMe}_{3}$, substitution of $\mathrm{MeNC}$ in $\left[\left(\mathrm{Ph}_{2} \mathrm{C}_{2} \mathrm{~S}_{2}\right) \mathrm{Ni}(\mathrm{CNMe})_{2}\right]$ leads only to $\left[\left(\mathrm{Ph}_{2} \mathrm{C}_{2} \mathrm{~S}_{2}\right) \mathrm{Ni}(\mathrm{CNMe})\left(\mathrm{PPh}_{3}\right)\right]$ (9) and $\left[\left(\mathrm{Ph}_{2} \mathrm{C}_{2} \mathrm{~S}_{2}\right) \mathrm{Ni}(\mathrm{CNMe})\left(\mathrm{PMe}_{3}\right)_{2}\right]$ (10), both of which have been identified spectroscopically and structurally by X-ray diffraction. With the kinetic advantage of the chelate effect, 1,2-bis(diphenylphosphino)ethane (dppe) effects a second displacement of MeNC and produces [( $\left.\left.\mathrm{Ph}_{2} \mathrm{C}_{2} \mathrm{~S}_{2}\right) \mathrm{Ni}(\mathrm{dppe})\right]$ (14). An enhanced $\mathrm{M}-\mathrm{CNMe}$ interaction is clearly manifested by a Ni-CNMe bond length that is shortened by $\sim 0.03 \AA$ relative to that in $\mathbf{4 a}$ ( $c f$. Tables 1 and $\mathbf{4}$ ) and by the greater resistance of this second isonitrile ligand to a second ligand substitution. As anticipated in view of the outcome found with $\mathrm{PPh}_{3}$, use of 1,4-bis(diphenylphosphino)benzene with $\left[\left(\mathrm{Ph}_{2} \mathrm{C}_{2} \mathrm{~S}_{2}\right) \mathrm{M}(\mathrm{CNMe})_{2}\right]$ yields dimetallic $\left[\left(\mathrm{Ph}_{2} \mathrm{C}_{2} \mathrm{~S}_{2}\right) \mathrm{M}(\mathrm{CNMe})\right]_{2}\left(\mu-1,4-\left(\mathrm{Ph}_{2} \mathrm{P}\right)_{2} \mathrm{C}_{6} \mathrm{H}_{4}\right)\left(\mathrm{M}=\mathrm{Ni}^{2+}(11), \mathrm{Pd}^{2+}(12), \mathrm{Pt}^{2+}(13)\right)$ (Scheme 5, Figure 1). Although crystals of 11-13 were grown under the same conditions, $\mathbf{1 1}$ crystallizes on a general position in triclinic $P$-1 with a syn disposition of the MeNC ligands, while 12 and 13 both occur on an inversion center in $P-1$, which demands an anti configuration of the two MeNC ligands. An upshot of the syn configuration in $\mathbf{1 1}$ is an intermetal distance that is $\sim 1.3 \AA$ shorter that in 12 and $\mathbf{1 3}$ (Table 4), while a consequence of the occurrence of $\mathbf{1 2}$ and $\mathbf{1 3}$ on inversion centers is a parallel disposition of the $\mathrm{S}_{2} \mathrm{MPC}$ mean planes that contrasts with an angle of $67.4^{\circ}$ for the square planar ends in $\mathbf{1 1}$. 
Table 4. Selected interatomic distances and angles in phosphine MeNC complexes.

\begin{tabular}{c|c|c|c|c|c}
\hline & $\mathbf{9}$ & $\mathbf{1 0}$ & $\mathbf{1 1}$ & $\mathbf{1 2}$ & $\mathbf{1 3}$ \\
\hline $\mathrm{M}-\mathrm{S}^{a}$ & $2.1407(5)$ & $2.1392(5)$ & $2.1345[6]^{i}$ & $2.2727(9)$ & $2.2780(10)$ \\
$\mathrm{M}-\mathrm{S}^{b}$ & $2.1497(5)$ & $2.1602(5)$ & $2.1463[6]$ & $2.2851(9)$ & $2.2901(10)$ \\
$\Delta_{\mathrm{M}-\mathrm{S}}$ & 0.009 & 0.021 & 0.0118 & 0.0124 & 0.0121 \\
$\mathrm{M}-\mathrm{P}$ & $2.2025(5)$ & $2.1908(5)$ & $2.2028[6]$ & $2.3210(9)$ & $2.2881(10)$ \\
$\mathrm{M}-\mathrm{CNMe}$ & $1.8233(19)$ & $1.8213(18)$ & $1.826[2]$ & $1.982(4)$ & $1.958(4)$ \\
$\mathrm{C} \equiv \mathrm{N}$ & $1.155(3)$ & $1.148(2)$ & $1.153[3]$ & $1.138(5)$ & $1.130(5)$ \\
$\mathrm{S}-\mathrm{M}-\mathrm{S}$ & $90.780(19)$ & $91.045(18)$ & $90.57[2]$ & $88.00(3)$ & $88.02(3)$ \\
$\mathrm{C}-\mathrm{M}-\mathrm{P}$ & $93.36(6)$ & $90.72(6)$ & $91.16[6]$ & $92.15(10)$ & $90.62(11)$ \\
$\mathrm{S}-\mathrm{M}-\mathrm{CNMe} \mathrm{C}^{c}$ & $167.92(6)$ & $177.74(6)$ & $177.85[7]$ & $176.20(10)$ & $177.18(11)$ \\
$\mathrm{S}-\mathrm{M}-\mathrm{P}^{c}$ & $90.576(19)$ & $89.737(19)$ & $90.76[2]$ & $91.46(3)$ & $91.66(3)$ \\
$\mathrm{S}-\mathrm{M}-\mathrm{CNMe}{ }^{d}$ & $88.06(6)$ & $88.78(6)$ & $87.54[6]$ & $88.53(10)$ & $89.86(11)$ \\
$\mathrm{S}-\mathrm{M}-\mathrm{P}^{d}$ & $166.47(2)$ & $172.788(19)$ & $177.07[3]$ & $174.31(3)$ & $174.95(3)$ \\
$\delta,{ }^{e} \AA$ & 0.187 & 0.078 & $0.010,0.047$ & 0.062 & 0.059 \\
$\tau,{ }^{f} \AA$ & - & - & 7.848 & 9.138 & 9.162 \\
$\theta,{ }^{g}$ deg. & 17.7 & 7.5 & $1.1,4.9$ & 5.7 & 5.4 \\
$\varphi,{ }^{h}$ deg. & - & - & 67.4 & 0.0 & 0.0 \\
\hline
\end{tabular}

${ }^{a} \mathrm{~S}$ atom trans to MeNC; ${ }^{b} \mathrm{~S}$ atom trans to phosphorus; ${ }^{c}$ Angle defined with $\mathrm{S}$ atom trans to MeNC; ${ }^{d}$ Angle defined with $\mathrm{S}$ atom cis to MeNC; ${ }^{e}$ Average atom

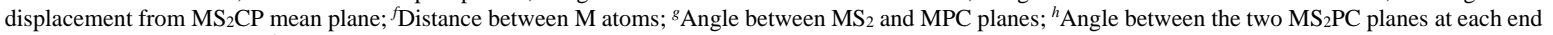
of the dimetallic molecule. ${ }^{i}[]$ notation designates uncertainties propagated in averaging.

Displacement of $\mathrm{MeNC}$ in $\left[\left(\mathrm{Ph}_{2} \mathrm{C}_{2} \mathrm{~S}_{2}\right) \mathrm{Ni}(\mathrm{CNMe})_{2}\right]$ by $\mathrm{CN}^{-}$can occur twice to afford $\left[\left(\mathrm{Ph}_{2} \mathrm{C}_{2} \mathrm{~S}_{2}\right) \mathrm{Ni}(\mathrm{CN})_{2}\right]^{2-}[\mathbf{1 5}]^{2-}$, but in poor yields of $\sim 9 \%$. The same dianion, however, is accessible in $\sim 34 \%$ yield in the reaction between $\left[\mathrm{Ni}(\mathrm{CN})_{4}\right]^{2-}$ and $\left[\left(\mathrm{Ph}_{2} \mathrm{C}_{2} \mathrm{~S}_{2}\right) \mathrm{Ni}(\mathrm{CNMe})_{2}\right]$. With $\left[\left(\mathrm{Ph}_{2} \mathrm{C}_{2} \mathrm{~S}_{2}\right) \mathrm{M}(\mathrm{CNMe})_{2}\right](\mathrm{M}=$ Pd or Pt), only monosubstitution to $\left[\left(\mathrm{Ph}_{2} \mathrm{C}_{2} \mathrm{~S}_{2}\right) \mathrm{M}(\mathrm{CN})(\mathrm{CNMe})\right]^{1-}$ occurs $\left(\mathrm{M}=\mathrm{Pd},[\mathbf{1 6}]^{1-} ; \mathrm{M}=\mathrm{Pt},\left[\mathbf{1 7}^{1-}\right)\right.$, evidently because enhanced dipositive character for the second and third metals provides for stronger metalligand bonding. Although no appreciable difference in M-CNMe bonds lengths exists between 5a vs [16 $]^{1-}$ or 6a vs $[\mathbf{1 7}]^{1-}$, the M-S bond lengths in $[\mathbf{1 6}]^{1-}$ and $[\mathbf{1 7}]^{1-}$ reveal a modest difference of $\sim 0.02 \AA$ (Table 5) that suggests a slightly stronger trans influence for $\mathrm{CN}^{-}$over CNMe.

Compounds 4a and 5a undergo substitution of CNMe by $\operatorname{IPr}$ (IPr = 1,3-bis(2,6diisopropylphenyl)imidazol-2-ylidene) to afford [( $\left.\left.\mathrm{Ph}_{2} \mathrm{C}_{2} \mathrm{~S}_{2}\right) \mathrm{M}(\mathrm{IPr})(\mathrm{CNMe})\right](\mathrm{M}=\mathrm{Ni}, \mathbf{1 8}$; $\mathrm{M}=\mathrm{Pd}$ 19), the structures of which reveal a difference of 0.011-0,020 $\AA$ between M-S bond lengths (Table 5). This difference is in accord with a documented ligand field strength for Arduengo-type carbene ligands that is at least comparable to that of phosphines and $\mathrm{CN}^{1-}$. Instead of simple ligand substitution, reaction between 6a and IPr leads unexpectedly to a ketenimine-type ligand formed by attachment of the carbene carbon to the isonitrile carbon. The $\mathrm{C}-\mathrm{C}_{\text {ketenimine }}$ bond length of $1.490[8] \AA$, which is indicative of single bond character, and an intraligand $\mathrm{C}-\mathrm{C}-\mathrm{N}$ angle of $113.0[4]^{\circ}$, which points toward $s p^{2}$ character for the carbon atom bound to Pt, are indicative of the charge separated resonance form of the ketenimine (Scheme 6, (b)) being the dominant contributor to its description. Insofar as $\Delta_{\mathrm{Pt}-\mathrm{S}}$ can be a gauge, (Table 5), the ketenimine ligand appears to be an appreciably stronger field ligand than even the Arduengo carbene. We are not aware of any prior example of this type of ligand in this binding mode. As reported by Bielawski, ${ }^{28}$ the free 
Table 5. Selected interatomic distances and angles in $\mathrm{CN}^{1-}$, carbene, and ketenimine complexes.

\begin{tabular}{|c|c|c|c|c|c|}
\hline & {$[16]^{1-}$} & {$[\mathbf{1 7}]^{1-}$} & 19 & $\mathbf{2 0}^{g}$ & 21 \\
\hline $\mathrm{M}-\mathrm{S}^{a}$ & $2.2605(7)$ & $2.2716(7)$ & $2.2697(14)$ & $2.272[1]$ & $2.1292(8)$ \\
\hline $\mathrm{M}-\mathrm{S}^{b}$ & $2.2821(7)$ & $2.2908(7)$ & $2.2808(13)$ & $2.317[1]$ & $2.1625(8)$ \\
\hline$\Delta_{\mathrm{M}-\mathrm{S}}$ & 0.0216 & 0.0192 & 0.0111 & 0.045 & 0.033 \\
\hline $\mathrm{M}-\mathrm{CN}, \mathrm{M}-\mathrm{C}_{\text {carbene/ketenimine }}$ & $2.019(2)$ & $1.993(2)$ & $2.046(5)$ & $2.057[6]$ & $1.906(3)$ \\
\hline $\mathrm{M}-\mathrm{CNMe}$ & $1.996(2)$ & 1.953(3) & $1.997(6)$ & $1.948[6]$ & $1.830(3)$ \\
\hline $\mathrm{C} \equiv \mathrm{NMe}$ & 1.131(3) & $1.140(3)$ & $1.127(7)$ & $1.135[7]$ & $1.148(4)$ \\
\hline $\mathrm{C}-\mathrm{C}_{\text {ketenimine }}$ & - & 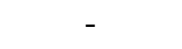 & & $1.490[8]$ & - \\
\hline S-M-S & 88.911(16) & $88.858(18)$ & $88.29(5)$ & $88.86[5]$ & $90.79(3)$ \\
\hline C-M-C & $92.50(7)$ & $92.08(8)$ & $92.9(5)$ & $92.7[2]$ & 89.47(13) \\
\hline S-M-CNMe ${ }^{c}$ & $176.81(5)$ & $177.86(7)$ & 174.21(16) & $178.3[2]$ & $174.77(10)$ \\
\hline $\mathrm{S}-\mathrm{M}-\mathrm{CN}^{c}$ & $177.78(6)$ & $176.70(6)$ & - & - & - \\
\hline $\mathrm{S}-\mathrm{M}-\mathrm{C}_{\text {carbene/ketenimine }}{ }^{\mathrm{c}}$ & - & - & 179.59(15) & $175.4[1]$ & $175.96(9)$ \\
\hline S-M-CNMe ${ }^{d}$ & $90.42(5)$ & $90.97(6)$ & $86.72(16)$ & $90.9[1]$ & 94.41(9) \\
\hline $\mathrm{S}-\mathrm{M}-\mathrm{CN}^{d}$ & $88.22(5)$ & $88.14(6)$ & - & - & - \\
\hline $\mathrm{S}-\mathrm{M}-\mathrm{C}_{\text {carbene/ketenimine }}{ }^{d}$ & - & - & $92.09(14)$ & 87.8[1] & 85.32(9) \\
\hline $\mathrm{C}-\mathrm{C}-\mathrm{N}_{\text {ketenimine }}$ & - & - & - & $113.0[4]$ & - \\
\hline $\mathrm{C}-\mathrm{N}-\mathrm{CH}_{3, \text { ketenimine }}$ & - & - & - & 118.1[5] & - \\
\hline$\delta,{ }^{e} \AA$ & 0.0267 & 0.0266 & 0.0252 & $0.0371,0.0396$ & 0.0064 \\
\hline$\theta,{ }^{\prime}$ deg. & 2.5 & 2.5 & 2.9 & 3.4, 3.7 & 1.2 \\
\hline
\end{tabular}

${ }^{a} \mathrm{~S}$ atom trans to MeNC; ${ }^{b} \mathrm{~S}$ atom trans to $\mathrm{NC}^{1-}$ or carbene; ${ }^{c}$ Angle defined with $\mathrm{S}$ atom trans to MeNC, $\mathrm{NC}^{1-}$, or carbene; ${ }^{d}$ Angle defined with $\mathrm{S}$ atom cis to MeNC, $\mathrm{CN}^{1-}$, carbene, or ketenimine; ${ }^{e}$ Average atom displacement from $\mathrm{MS}_{2} \mathrm{CP}$ mean plane; ${ }^{f}$ Angle between $\mathrm{MS}_{2}$ and $\mathrm{MC}_{2}$ planes; ${ }^{9} \mathrm{Two}$ independent molecules occur in the asymmetric unit. Values are averages of corresponding values from both molecules, and [] notation designates uncertainties propagated in averaging.

ketenimine that would be obtained by reaction of $\mathrm{C} \equiv \mathrm{N}-{ }^{t} \mathrm{Bu}$ and 1,3-bis(2,4,6-trimethylphenyl)imidazol-2ylidene results in a similar angled geometry but faces unfavorable activation energetics. Carbene-derived ketenimines arising from direct reaction between isonitrile and carbene only appear accessible from electronically perturbed carbene derivatives such as $N, N^{\prime}$-diamido substituted variants. ${ }^{28}$

Use of tetraisopropylbenzobisimidazolium dibromide (Scheme 5), intended as a precursor to a bridging bis(carbene), in reaction with 4a produced an unanticipated Fischer carbene ligand wherein the carbenoid carbon is asymmetrically flanked by $\mathrm{NMe}_{2}$ and NHMe groups. The probable genesis of this ligand is via condensation of a free $\mathrm{C} \equiv \mathrm{NMe}$ molecule with bound $\mathrm{C} \equiv \mathrm{NMe}$, with hydrogen atoms possibly originating from incompletely deprotected benzobisimidazolium dication or from adventitious moisture. In light of the

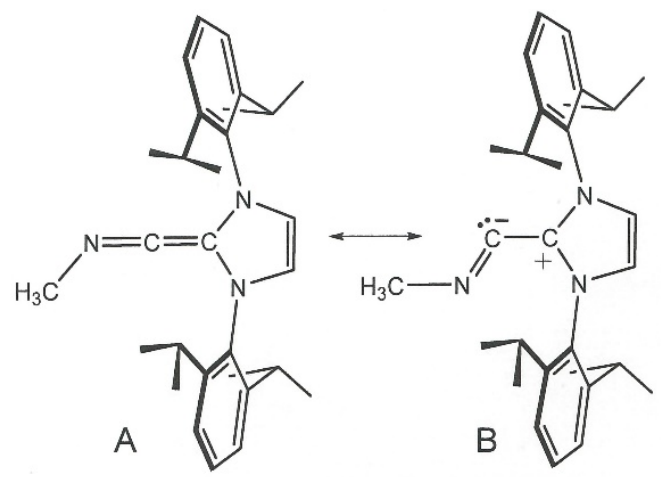

Scheme 6. Resonance forms of a ketenimine. 


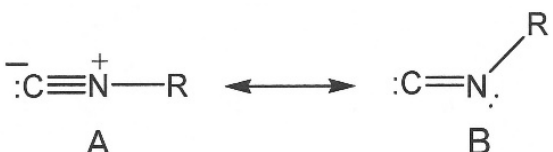

Scheme 7. Resonance forms of an isonitrile ligand.

well-documented capacity of carbenes to catalytically mediate a broad variety of carbon-carbon bond forming reactions, ${ }^{29}$ this unanticipated outcome may involve the bis(carbene) complex activating either bound or free MeNC in a direct reaction, as opposed to accomplishing the intended straightforward substitution reaction. As gauged by the $\Delta_{\mathrm{M}-\mathrm{S}}$ values, the carbene ligand in $\mathbf{2 1}$ exerts a somewhat stronger trans influence than IPr, possibly because the larger steric profile of the latter tends to limit its bonding distance to $\mathrm{M}^{2+}$. The asymmetry to the carbene ligand in $\mathbf{2 1}$ appears not to have a precedent, at least not in a structurally authenticated compound, but the symmetric noncyclic $\mathrm{C}\left(\mathrm{NMe}_{2}\right)_{2}$ carbene has been used in a few instances as a ligand in Group 11 complexes. ${ }^{30-31}$

Spectroscopy. Compounds sets 4-6 exhibit two infrared stretching frequencies in the $2100-2300 \mathrm{~cm}^{-1}$ region, which arise from the symmetric and antisymmetric vibrations of the $\mathrm{C} \equiv \mathrm{NR}$ pair. These frequencies occur at higher energy than found for the free isonitrile (Table 6), indicating that $\pi$-backbonding is not

Table 6. Summary of infrared $\mathrm{C} \equiv \mathrm{N}$ stretching frequencies $\left(\mathrm{cm}^{-1}\right)$ in $\mathrm{CH}_{2} \mathrm{Cl}_{2}$ solution.

\begin{tabular}{|c|c|c|c|}
\hline & $\mathrm{Ni}$ & Pd & Pt \\
\hline$\left[(\mathrm{pdt}) \mathrm{M}(\mathrm{CNMe})_{2}\right]^{a}(2168)^{\mathrm{b}}$ & 2228,2214 & 2246,2230 & 2248,2223 \\
\hline$\left[(\mathrm{pdt}) \mathrm{M}(\mathrm{CNBn})_{2}\right](2155)^{b}$ & 2213, 2197 & 2227, 2213 & 2229, 2204 \\
\hline$\left[(\mathrm{pdt}) \mathrm{M}(\mathrm{CNCy})_{2}\right](2144)^{b}$ & 2204, 2189 & 2217, 2203 & 2219, 2196 \\
\hline$\left[(\mathrm{pdt}) \mathrm{M}\left(\mathrm{CN}^{t} \mathrm{Bu}\right)_{2}\right](2140)^{b}$ & 2197, 2180 & 2213, 2197 & 2214, 2188 \\
\hline$\left[(\mathrm{pdt}) \mathrm{M}(\mathrm{CN}-1-\mathrm{Ad})_{2}\right](2133)^{b}$ & 2196, 2175 & 2211, 2198 & 2212, 2192 \\
\hline$\left[(\mathrm{pdt}) \mathrm{M}(\mathrm{CNPh})_{2}\right](2130)^{b}$ & 2182, 2162 & 2198, 2182 & 2199, 2172 \\
\hline$\left[(\mathrm{adt}) \mathrm{M}\left(\mathrm{CN}^{t} \mathrm{Bu}\right)_{2}\right]^{c}$ & 2196, 2180 & - & - \\
\hline$\left[(\mathrm{mdt}) \mathrm{M}\left(\mathrm{CN}^{t} \mathrm{Bu}\right)_{2}\right]^{d}$ & 2196, 2177 & - & - \\
\hline$\left[(\mathrm{pdt}) \mathrm{M}\left(\mathrm{CN}^{t} \mathrm{Bu}\right)_{2}\right]^{1+}$ & - & - & 2243, 2222 \\
\hline$\left[\left((\mathrm{pdt}) \mathrm{M}\left(\mathrm{CN}^{t} \mathrm{Bu}\right)_{2}\right)_{2}(\mu-\mathrm{Ag})_{2}\right]^{2+}$ & 2211, 2198 & - & - \\
\hline [(pdt)M(CNMe)(PMe $)]$ & 2199 & - & - \\
\hline$\left[(\mathrm{pdt}) \mathrm{M}(\mathrm{CNMe})\left(\mathrm{PPh}_{3}\right)\right]$ & 2204 & - & - \\
\hline$[(\mathrm{pdt}) \mathrm{M}(\mathrm{CNMe})]_{2}(\mu-1,4-\mathrm{dppb})$ & $2223,{ }^{e} 2211$ & $2240,{ }^{e} 2227$ & $2237,,^{e} 2222$ \\
\hline$\left[(\mathrm{pdt}) \mathrm{M}(\mathrm{CN})_{2}\right]^{2-}$ & $2100,2095^{f}$ & - & - \\
\hline$[(\mathrm{pdt}) \mathrm{M}(\mathrm{CN})(\mathrm{CNMe})]^{1-}$ & - & 2227, 2120 & 2219, 2120 \\
\hline$[(\mathrm{pdt}) \mathrm{M}(\mathrm{IPr})(\mathrm{CNMe})]$ & $2190^{f}$ & 2213 & - \\
\hline$[(\mathrm{pdt}) \mathrm{M}(\mathrm{C}(\mathrm{NMe})(\mathrm{IPr}))(\mathrm{CNMe})]$ & - & - & 2213 \\
\hline$[(\mathrm{pdt}) \mathrm{M}(\mathrm{C}(\mathrm{NMe})(\mathrm{IPr}))(\mathrm{CNMe})]$ & 2197 & - & - \\
\hline
\end{tabular}



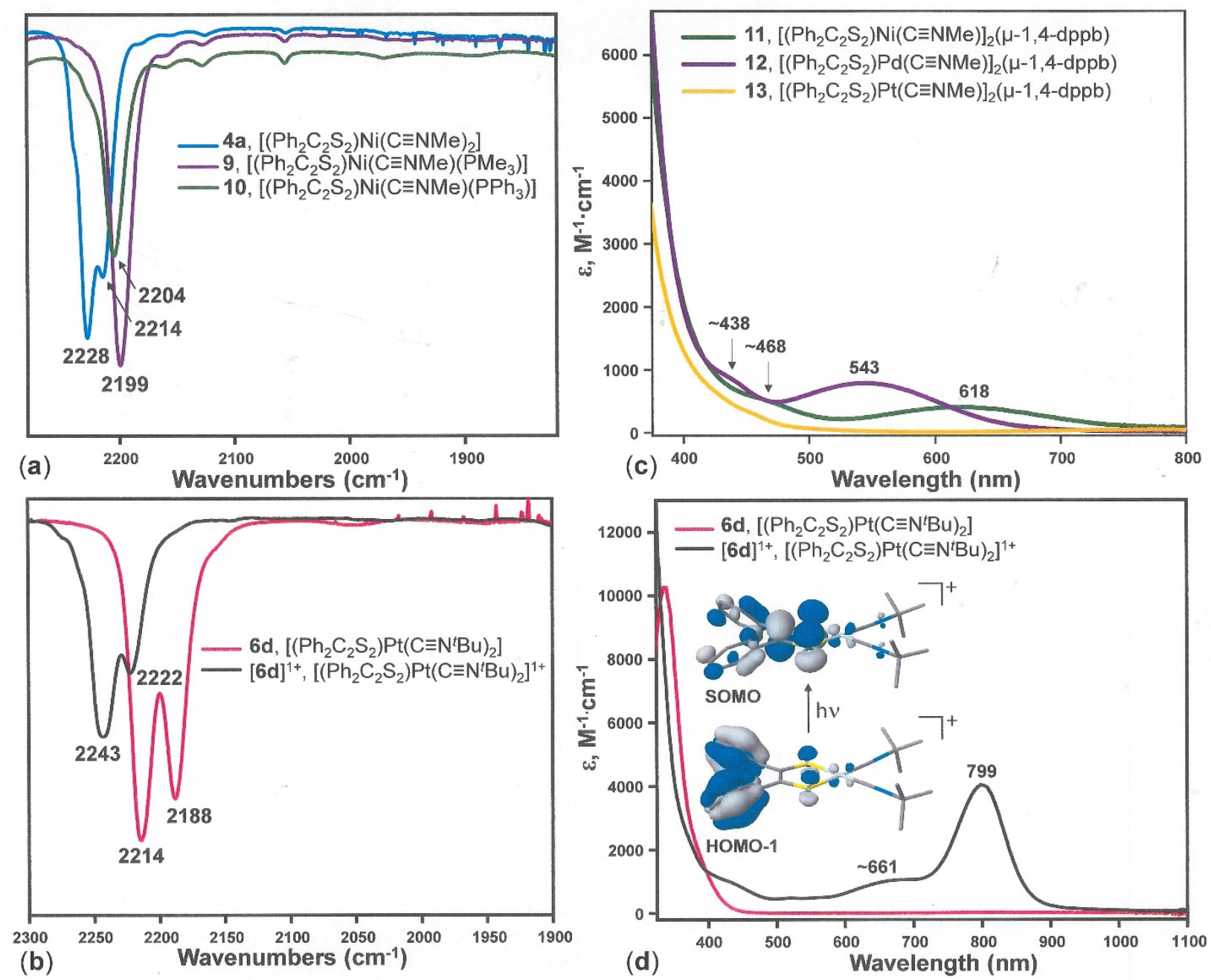

Figure 2. Selected IR ((a) and (b)) and UV-vis ((c) and (d)) spectra. The inset to panel (d) illustrates the MOs involved (0.04 contour level) with the $799 \mathrm{~nm}$ absorption.

relevant to the metal-ligand interaction and suggesting instead that the increase in stretching frequency arises from an increased importance of resonance form A relative to B (Scheme 7) when ligated to metal. These stretching frequencies increase as one traverses the series from $\mathrm{Ni}^{2+}$ to $\mathrm{Pd}^{2+}$ to $\mathrm{Pt}^{2+}$ at constancy of ligand, implying then an increasing positive character to the metal ion such that resonance form $\mathrm{A}$ is even further accentuated in its weighted contribution to the electronic description. A similar effect has been noted by Cotton in Group 10 isonitrile complexes. ${ }^{32}$ This behavior contrasts sharply with the $173-183 \mathrm{~cm}^{-1}$ shift to lower energy observed for $v_{\mathrm{CN}}$ in the zerovalent homoleptic isonitrile compounds $[\mathrm{M}(\mathrm{CN}(2,6-$ $\left.\left.\left.\mathrm{Me}_{2} \mathrm{C}_{6} \mathrm{H}_{3}\right)\right)_{6}\right]\left(\mathrm{M}=\mathrm{V},{ }^{33} \mathrm{~W}^{34}\right)$ and $\left[\mathrm{M}\left(\mathrm{CN}\left(2,6-{ }^{i} \mathrm{Pr}_{2} \mathrm{C}_{6} \mathrm{H}_{3}\right)\right)_{6}\right]\left(\mathrm{M}=\mathrm{Nb},{ }^{35} \mathrm{Ta},{ }^{36} \mathrm{~W}^{37}\right)$, as compared to the free ligands. A decrease in $v_{\mathrm{CN}}$ is found for 4-6 as $\mathrm{R}$ becomes more electron-donating $\left(\mathrm{Me}>\mathrm{Bn}>\mathrm{Cy}>{ }^{t} \mathrm{Bu} \approx\right.$ 1-Ad), while Ph produces the lowest value for $v_{\mathrm{CN}}$ through a presumed delocalizing effect upon the isonitrile $\pi$-electrons. 
Although $\pi$-backbonding does not describe the metal-isonitrile interaction in the compounds of Schemes 3 and 4, the isonitrile frequencies in $\left[\left(\mathrm{Ph}_{2} \mathrm{C}_{2} \mathrm{~S}_{2}\right) \mathrm{M}(\mathrm{CNMe}) \mathrm{L}\right]$ complexes manifest variation that apparently correlates to the $\sigma$ donor power of L (Figure 2 (a)). Stronger $\sigma$-donors should moderate the positive character of $\mathrm{M}^{2+}$ and, because the negative end in charge-separated form $\mathrm{A}$ is then joined to metal ion with attenuated cationic charge, they should tend to promote resonance form B over A. The shift from 2204 to 2199 to $2190 \mathrm{~cm}^{-1}$ for $v_{\mathrm{CN}}$ in moving from $\left[\left(\mathrm{Ph}_{2} \mathrm{C}_{2} \mathrm{~S}_{2}\right) \mathrm{Ni}(\mathrm{CNMe})\left(\mathrm{PPh}_{3}\right)\right]$ to $\left[\left(\mathrm{Ph}_{2} \mathrm{C}_{2} \mathrm{~S}_{2}\right) \mathrm{Ni}(\mathrm{CNMe})\left(\mathrm{PMe}_{3}\right)\right]$ to $\left[\left(\mathrm{Ph}_{2} \mathrm{C}_{2} \mathrm{~S}_{2}\right) \mathrm{Ni}(\mathrm{CNMe})(\mathrm{IPr})\right]$ has a plausible basis in this light. In contrast,

Table 7. Summary of UV-vis spectral data for all compounds.

\begin{tabular}{|c|c|}
\hline Compound & Electronic spectra, $\lambda_{\max }, \mathrm{nm}\left(\varepsilon_{\mathrm{M}}, \mathrm{M}^{-1} \mathrm{~cm}^{-1}\right)$ \\
\hline$\left[\left(\mathrm{Ph}_{2} \mathrm{C}_{2} \mathrm{~S}_{2}\right) \mathrm{Ni}(\mathrm{CNMe})_{2}\right]^{a}$ & 274 (31700), 348 (17200), 445 (240), 604 (300) \\
\hline$\left[\left(\mathrm{Ph}_{2} \mathrm{C}_{2} \mathrm{~S}_{2}\right) \mathrm{Ni}(\mathrm{CNBn})_{2}\right]$ & $460(400), 608(420)$ \\
\hline$\left[\left(\mathrm{Ph}_{2} \mathrm{C}_{2} \mathrm{~S}_{2}\right) \mathrm{Ni}(\mathrm{CNCy})_{2}\right]$ & 440 (240), $601(420)$ \\
\hline$\left[\left(\mathrm{Ph}_{2} \mathrm{C}_{2} \mathrm{~S}_{2}\right) \mathrm{Ni}\left(\mathrm{CN}^{t} \mathrm{Bu}\right)_{2}\right]$ & 424 (260), $600(410)$ \\
\hline$\left.\left[\left(\mathrm{MeO}-p-\mathrm{C}_{6} \mathrm{H}_{4}\right)_{2} \mathrm{C}_{2} \mathrm{~S}_{2}\right) \mathrm{Ni}\left(\mathrm{CN}^{t} \mathrm{Bu}\right)_{2}\right]$ & 420 (200), 607 (320) \\
\hline$\left[\left(\mathrm{Me}_{2} \mathrm{C}_{2} \mathrm{~S}_{2}\right) \mathrm{Ni}\left(\mathrm{CN}^{t} \mathrm{Bu}\right)_{2}\right]$ & 350 (6400), 450 (290), 614 (170) \\
\hline$\left[\left(\mathrm{Ph}_{2} \mathrm{C}_{2} \mathrm{~S}_{2}\right) \mathrm{Ni}(\mathrm{CN}-1-\mathrm{Ad})_{2}\right]$ & 437 (180), $599(360)$ \\
\hline$\left[\left(\mathrm{Ph}_{2} \mathrm{C}_{2} \mathrm{~S}_{2}\right) \mathrm{Ni}(\mathrm{CNPh})_{2}\right]$ & 466 (1400), 639 (480) \\
\hline$\left[\left(\mathrm{Ph}_{2} \mathrm{C}_{2} \mathrm{~S}_{2}\right) \mathrm{Pd}(\mathrm{CNMe})_{2}\right]$ & 421 (690), 502 (530) \\
\hline$\left[\left(\mathrm{Ph}_{2} \mathrm{C}_{2} \mathrm{~S}_{2}\right) \mathrm{Pd}(\mathrm{CNBn})_{2}\right]$ & 427 (150), 518 (50) \\
\hline$\left[\left(\mathrm{Ph}_{2} \mathrm{C}_{2} \mathrm{~S}_{2}\right) \mathrm{Pd}(\mathrm{CNCy})_{2}\right]$ & 431 (430), 504 (490) \\
\hline$\left[\left(\mathrm{Ph}_{2} \mathrm{C}_{2} \mathrm{~S}_{2}\right) \mathrm{Pd}\left(\mathrm{CN}^{t} \mathrm{Bu}\right)_{2}\right]^{a}$ & 240 (32500), 336 (17300), 419 (860), 498 (620) \\
\hline$\left[\left(\mathrm{Ph}_{2} \mathrm{C}_{2} \mathrm{~S}_{2}\right) \mathrm{Pd}(\mathrm{CN}-1-\mathrm{Ad})_{2}\right]$ & $430(320), 505(420)$ \\
\hline$\left[\left(\mathrm{Ph}_{2} \mathrm{C}_{2} \mathrm{~S}_{2}\right) \mathrm{Pd}(\mathrm{CNPh})_{2}\right]$ & 385 (5180), 534 (770) \\
\hline$\left[\left(\mathrm{Ph}_{2} \mathrm{C}_{2} \mathrm{~S}_{2}\right) \mathrm{Pt}(\mathrm{CNMe})_{2}\right]$ & $337(4780)$ \\
\hline$\left[\left(\mathrm{Ph}_{2} \mathrm{C}_{2} \mathrm{~S}_{2}\right) \mathrm{Pt}(\mathrm{CNBn})_{2}\right]$ & $342(1560)$ \\
\hline$\left[\left(\mathrm{Ph}_{2} \mathrm{C}_{2} \mathrm{~S}_{2}\right) \mathrm{Pt}(\mathrm{CNCy})_{2}\right]$ & $336(9970)$ \\
\hline$\left[\left(\mathrm{Ph}_{2} \mathrm{C}_{2} \mathrm{~S}_{2}\right) \mathrm{Pt}\left(\mathrm{CN}^{t} \mathrm{Bu}\right)_{2}\right]$ & $336(10290)$ \\
\hline$\left[\left(\mathrm{Ph}_{2} \mathrm{C}_{2} \mathrm{~S}_{2}\right) \mathrm{Pt}(\mathrm{CN}-1-\mathrm{Ad})_{2}\right]$ & $336(6850)$ \\
\hline$\left[\left(\mathrm{Ph}_{2} \mathrm{C}_{2} \mathrm{~S}_{2}\right) \mathrm{Pt}(\mathrm{CNPh})_{2}\right]^{a}$ & 274 (35800), 390 (4070) \\
\hline$\left[\left(\mathrm{Ph}_{2} \mathrm{C}_{2} \mathrm{~S}_{2}\right) \mathrm{Pt}\left(\mathrm{CN}^{t} \mathrm{Bu}\right)_{2}\right]^{1+}$ & 439 (910), 661 (1030), 799 (4050) \\
\hline$\left[\left(\left(\mathrm{Ph}_{2} \mathrm{C}_{2} \mathrm{~S}_{2}\right) \mathrm{Ni}\left(\mathrm{CN}^{t} \mathrm{Bu}\right)_{2}\right)_{2}(\mu-\mathrm{Ag})_{2}\right]^{2+}$ & $523(\sim 35)$ \\
\hline$\left[\left(2,6-\mathrm{Me}_{2} \mathrm{py}\right) \mathrm{Ni}\left(\mu_{2}-\eta^{1}, \eta^{1}-\mathrm{S}^{\prime}, \eta^{1}-\mathrm{S}^{\prime \prime}-\mathrm{C}_{2} \mathrm{Ph}_{2}\right)\right]_{2}$ & 272 (8670), 364 (2800), 482 (2230), 693 (260) \\
\hline$\left[\left(\left(\mathrm{Ph}_{2} \mathrm{C}_{2} \mathrm{~S}_{2}\right) \mathrm{Ni}(\mathrm{C} \equiv \mathrm{NMe})\right)_{2}(\mu-\mathrm{dppb})\right]$ & $468(520), 618(420)$ \\
\hline$\left[\left(\left(\mathrm{Ph}_{2} \mathrm{C}_{2} \mathrm{~S}_{2}\right) \mathrm{Pd}(\mathrm{C} \equiv \mathrm{NMe})\right)_{2}(\mu-\mathrm{dppb})\right]$ & 438 (890), 543 (820) \\
\hline$\left[\left(\left(\mathrm{Ph}_{2} \mathrm{C}_{2} \mathrm{~S}_{2}\right) \mathrm{Pt}(\mathrm{C} \equiv \mathrm{NMe})\right)_{2}(\mu-\mathrm{dppb})\right]$ & $340(11800)$ \\
\hline$\left[\left(\mathrm{Ph}_{2} \mathrm{C}_{2} \mathrm{~S}_{2}\right) \mathrm{Ni}(\mathrm{CNMe})\left(\mathrm{PMe}_{3}\right)\right]$ & $564(370)$ \\
\hline$\left[\left(\mathrm{Ph}_{2} \mathrm{C}_{2} \mathrm{~S}_{2}\right) \mathrm{Ni}(\mathrm{CNMe})\left(\mathrm{PPh}_{3}\right)\right]$ & 348 (3900), 463 (210), 622 (190) \\
\hline$\left[\left(\mathrm{Ph}_{2} \mathrm{C}_{2} \mathrm{~S}_{2}\right) \mathrm{Ni}(\mathrm{CN})_{2}\right]^{2-}$ & 489 (220), 378 (5380) \\
\hline$\left[\left(\mathrm{Ph}_{2} \mathrm{C}_{2} \mathrm{~S}_{2}\right) \mathrm{Pd}(\mathrm{CN})(\mathrm{CNMe})\right]^{1-}$ & 338 (9800), 553 (920) \\
\hline$\left[\left(\mathrm{Ph}_{2} \mathrm{C}_{2} \mathrm{~S}_{2}\right) \mathrm{Pt}(\mathrm{CN})(\mathrm{CNMe})\right]^{1-}$ & $497(1350)$ \\
\hline$\left[\left(\mathrm{Ph}_{2} \mathrm{C}_{2} \mathrm{~S}_{2}\right) \mathrm{Ni}(\mathrm{IPr})(\mathrm{CNMe})\right]^{a}$ & 350 (11600), 560 (410), 867 (120) \\
\hline$\left[\left(\mathrm{Ph}_{2} \mathrm{C}_{2} \mathrm{~S}_{2}\right) \mathrm{Pd}(\mathrm{IPr})(\mathrm{CNMe})\right]$ & $470(370)$ \\
\hline$\left[\left(\mathrm{Ph}_{2} \mathrm{C}_{2} \mathrm{~S}_{2}\right) \mathrm{Pt}(\mathrm{C}(\mathrm{NMe})(\mathrm{IPr}))(\mathrm{CNMe})\right]$ & $344(7530)$ \\
\hline$\left[\left(\mathrm{Ph}_{2} \mathrm{C}_{2} \mathrm{~S}_{2}\right) \mathrm{N}(\mathrm{CNMe})\left(\mathrm{C}(\mathrm{NHMe})\left(\mathrm{NMe}_{2}\right)\right)\right]$ & $510(200)$ \\
\hline
\end{tabular}

${ }^{a}$ Data have been previously published. 


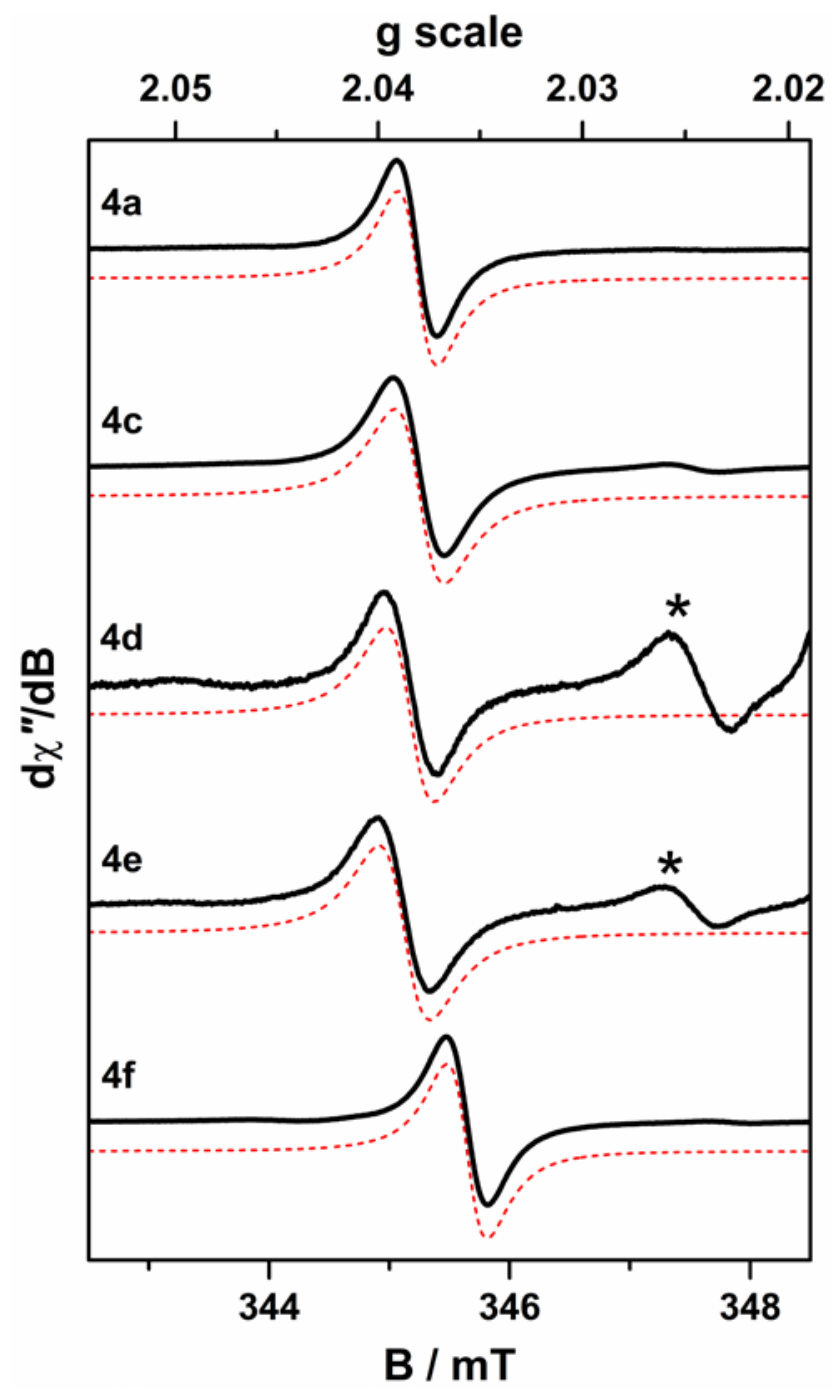

Figure 3. Comparison of the X-band EPR spectra of one-electron oxidized 4a and 4c-4f recorded in $\mathrm{CH}_{2} \mathrm{Cl}_{2}$ solution at $293 \mathrm{~K}$ (experimental conditions: frequency, $9.808 \mathrm{GHz}$; power, $6.3 \mathrm{~mW}$; modulation, $0.5 \mathrm{mT}$ ). Experimental data are represented by the black line; simulation is depicted by the dashed trace: $[4 \mathbf{a}]^{+}, g_{\text {iso }}=2.0381 ;[\mathbf{4 c}]^{+}, g_{\text {iso }}=2.0383 ;[4 \mathbf{d d}]^{+}, g_{\text {iso }}=2.0386 ;[\mathbf{4 e}]^{+}, g_{\text {iso }}=2.0386 ;[\mathbf{4 f}]^{+}, g_{\text {iso }}=2.0353$. The asterisk denotes an impurity caused by partial decomposition of the oxidized species at ambient temperature.

an effect of substantially greater magnitude is found for the $\left[(\mathrm{acac}) \mathrm{Rh}(\mathrm{CO})\left(\mathrm{PR}_{3}\right)\right]$ series $\mathrm{R}=\mathrm{Ph}, \mathrm{Cy}$, and Ad ( $v_{\mathrm{CO}}=1977.6,1958.7,1948.3 \mathrm{~cm}^{-1}$, respectively), where bona fide $\pi$-backbonding is likely operative. ${ }^{38-}$ ${ }^{39}$ For cationic $\left[\left(\mathrm{Ph}_{2} \mathrm{C}_{2} \mathrm{~S}_{2}\right) \mathrm{Pt}\left(\mathrm{CN}^{t} \mathrm{Bu}\right)_{2}\right]^{1+},[\mathbf{6 d}]^{1+}$, both symmetric and antisymmetric $v_{\mathrm{CN}}$ shift to higher energy by $\sim 30 \mathrm{~cm}^{-1}$ (Table 6, Figure 2 (b)) due to an amplification of the positive character at $\mathrm{Pt}^{2+}$ by oxidation of the dithiolene ligand to a monoanion and enhancement of structure A in Scheme 7. Structurally, in keeping with this increase in $v_{\mathrm{CN}},[\mathbf{6 d}]_{2}{ }^{2+}$ shows a decrease in its average $\mathrm{C} \equiv \mathrm{N}$ bond length by $\sim 0.014 \AA$ relative to $\mathbf{6 d}$ (Tables 1 and 2). A more modest increase in $v_{\mathrm{CN}}$ by $\sim 16 \mathrm{~cm}^{-1}$ is observed for $\left[\left(\left(\mathrm{Ph}_{2} \mathrm{C}_{2} \mathrm{~S}_{2}\right) \mathrm{Ni}\left(\mathrm{CN}^{t} \mathrm{Bu}\right)_{2}\right)_{2}(\mu-\mathrm{Ag})_{2}\right]^{2+}$ vs $\left[\left(\mathrm{Ph}_{2} \mathrm{C}_{2} \mathrm{~S}_{2}\right) \mathrm{Ni}\left(\mathrm{CN}^{t} \mathrm{Bu}\right)_{2}\right]$ since the positive charge, being associated with the $\mathrm{Ag}^{1+}$ ions, is less directly transmitted to the Group 10 metal. 
The UV-vis spectra of compounds 4 feature low intensity absorptions at $\sim 600 \mathrm{~nm}$ and at 420-460 nm, the former of which is a transition between the HOMO, comprised of the dithiolene $\pi$ system, and the LUMO, which is $\mathrm{M}-\mathrm{L} \sigma^{*}$ in nature and involves the metal $\mathrm{d}_{\mathrm{x}^{2}-\mathrm{y}^{2}}$ atomic orbital. This band shifts to higher energy when $\mathrm{M}=\operatorname{Pd}(\sim 500 \mathrm{~nm})$ and Pt (unresolved from other features at still higher energy) owing to the inherently higher energies of the d orbitals of the heavier metals (Table 7). The same pattern is evident in the mono(isonitrile) compounds of the type $\left[\left(\mathrm{Ph}_{2} \mathrm{C}_{2} \mathrm{~S}_{2}\right) \mathrm{M}(\mathrm{CNMe}) \mathrm{L}\right]$. As M varies from Ni to Pd to Pt in $\left[\left(\mathrm{Ph}_{2} \mathrm{C}_{2} \mathrm{~S}_{2}\right) \mathrm{M}(\mathrm{CNMe})\right]_{2}(\mu-1,4-\mathrm{dppb})$, for example, the lowest energy absorption similarly moves from 618 $\mathrm{nm}$ to $543 \mathrm{~nm}$ to a position unresolved from higher energy absorption above $450 \mathrm{~nm}$ (Figure 2, (c)).

Oxidation of $\mathbf{6 d}$ by one electron induces a dark color and the onset of an intense low energy maximum at $\sim 799 \mathrm{~nm}$ with an unresolved shoulder at $\sim 661 \mathrm{~nm}$ (Figure 2 (d)). Absorptions in the lower energy 900$1100 \mathrm{~nm}$ region are conspicuously absent. The related cation, $\left.\left[\left({ }^{t} \mathrm{Bu}-p-\mathrm{C}_{6} \mathrm{H}_{4}\right)_{2} \mathrm{C}_{2} \mathrm{~S}_{2}\right) \mathrm{Pt}\left(4,4^{\prime}-{ }^{t} \mathrm{Bu}_{2}-\mathrm{bipy}\right)\right]^{+}\left(\lambda_{\max }\right.$ $=728 \mathrm{~nm}$ ) has been shown by Wieghardt and coworkers to be in thermal equilibrium with the dimeric dication $\left[\left({ }^{t} \mathrm{Bu}-p-\mathrm{C}_{6} \mathrm{H}_{4}\right)_{2} \mathrm{C}_{2} \mathrm{~S}_{2}\right) \mathrm{Pt}\left(4,4^{\prime}-{ }^{t} \mathrm{Bu}_{2}-\right.$ bipy $\left.)\right]_{2}{ }^{2+}\left(\lambda_{\max }=1015 \mathrm{~nm}\right)$ and to be favored at ambient temperature. ${ }^{25}$ Considering the fundamental similarity between $\left[\left({ }^{t} \mathrm{Bu}-p-\mathrm{C}_{6} \mathrm{H}_{4}\right)_{2} \mathrm{C}_{2} \mathrm{~S}_{2}\right) \mathrm{Pt}\left(4,4^{\prime}-{ }^{t} \mathrm{Bu}_{2}-\right.$ bipy $\left.)\right]^{+}$and $\left[\left(\mathrm{Ph}_{2} \mathrm{C}_{2} \mathrm{~S}_{2}\right) \mathrm{Pt}\left(\mathrm{C} \equiv \mathrm{N}^{t} \mathrm{Bu}\right)_{2}\right]^{+}$, the lack of any absorption feature at energy lower than $800 \mathrm{~nm}$ suggests that $\left[\left(\mathrm{Ph}_{2} \mathrm{C}_{2} \mathrm{~S}_{2}\right) \mathrm{Pt}\left(\mathrm{C} \equiv \mathrm{N}^{t} \mathrm{Bu}\right)_{2}\right]^{+}$is the dominant species in solution and that $\left[\left[\left(\mathrm{Ph}_{2} \mathrm{C}_{2} \mathrm{~S}_{2}\right) \mathrm{Pt}\left(\mathrm{C} \equiv \mathrm{N}^{t} \mathrm{Bu}\right)_{2}\right]_{2}\right]^{2+}$, although the form identified in the crystalline state, is of negligible concentration in $\mathrm{CH}_{2} \mathrm{Cl}_{2}$ at $25^{\circ} \mathrm{C}$. Time-dependent DFT calculations upon $\left[\left(\mathrm{Ph}_{2} \mathrm{C}_{2} \mathrm{~S}_{2}\right) \mathrm{Pt}\left(\mathrm{C} \equiv \mathrm{N}^{t} \mathrm{Bu}\right)_{2}\right]^{+}$suggest that the $799 \mathrm{~nm}$ absorption is due to an intraligand dithiolene $\pi$ (phenyl) $\rightarrow \pi\left(\mathrm{C}_{2} \mathrm{~S}_{2}\right)$ excitation ((Figure 2 (d), inset) and that the $661 \mathrm{~nm}$ band is similar in description but involves the HOMO-2 with a different constitution to its phenyl $\pi$ character.

The one-electron oxidation generally observed for compound types 4-6 is predominantly based on the $\mathrm{C}_{2} \mathrm{~S}_{2} \pi$ system of the dithiolene ligand and produces a delocalized radical monoanion that gives rise to an isotropic signal. The $g$ value for this signal is subject to minor variation as function of the nature of the isonitrile substituent $\mathrm{R}$ (Figure 3). A slight shift to higher $g$ values is found as $\mathrm{R}$ varies from Me to the more electron-rich ${ }^{t} \mathrm{Bu}$ and 1-adamantyl, while a somewhat greater move to lower $g$ value is observed when $\mathrm{R}=\mathrm{Ph}$. As noted earlier in the context of $\mathrm{C} \equiv \mathrm{N}$ stretching frequencies, the $\mathrm{Ph} \pi$-system exerts a modest delocalizing influence upon the $\mathrm{C} \equiv \mathrm{N}$ multiple bond; indirectly therefrom, the Ph group registers more effect upon the dithiolene radical than its aliphatic counterparts.

Electrochemistry. Compounds sets 4, 5, and 6 display a reversible or quasireversible one-electron oxidation that is due to the $\mathrm{Ph}_{2} \mathrm{C}_{2} \mathrm{~S}_{2}{ }^{2-} \rightarrow \mathrm{Ph}_{2} \mathrm{C}_{2} \mathrm{~S}^{-} \mathrm{S}^{-}+\mathrm{e}^{-}$transformation. Consistent with the increasing positive character at metal that is indicated by $v_{\mathrm{CN}}$ stretching frequencies (vide supra), the potentials for these oxidations $\left(E_{\text {ox }}\right)$ trend toward more positive values as M varies from Ni to Pd to Pt (Table 8). Where available, data for $\left[\left(\mathrm{Ph}_{2} \mathrm{C}_{2} \mathrm{~S}_{2}\right) \mathrm{M}(\mathrm{CNMe}) \mathrm{L}\right]$ ( $\mathrm{L}=$ phosphine, carbene) show $E_{\mathrm{ox}}$ that move toward less positive values as the donor strength of $\mathrm{L}$ increases and renders the oxidation more facile. Thus, $E_{\mathrm{ox}}$ values 
Table 8. Summary of cyclic voltammetry data for $\left[(\mathrm{pdt}) \mathrm{M}\left(\mathrm{L}_{1}\right)\left(\mathrm{L}_{2}\right)\right]$ in $\mathrm{CH}_{2} \mathrm{Cl}_{2}$ with $\left[{ }^{n} \mathrm{Bu}_{4} \mathrm{~N}\right]\left[\mathrm{PF}_{6}\right]$ supporting electrolyte. All waves are reversible except where noted otherwise. Potentials are referenced with respect to $\mathrm{AgCl} / \mathrm{Ag}^{a}{ }^{a}$

\begin{tabular}{|c|c|c|c|c|}
\hline & & $\mathrm{M}=\mathrm{Ni}$ & $\mathrm{M}=\mathrm{Pd}$ & $\mathrm{M}=\mathrm{Pt}$ \\
\hline MeNC & MeNC & +0.52 & +0.61 & +0.64 \\
\hline $\mathrm{C}_{6} \mathrm{H}_{5} \mathrm{CH}_{2} \mathrm{NC}$ & $\mathrm{C}_{6} \mathrm{H}_{5} \mathrm{CH}_{2} \mathrm{NC}$ & +0.50 & +0.56 & +0.57 \\
\hline CyNC & CyNC & +0.56 & +0.61 & +0.67 \\
\hline${ }^{t} \mathrm{BuNC}$ & ${ }^{t} \mathrm{BuNC}$ & +0.59 & +0.60 & +0.66 \\
\hline${ }^{t} \mathrm{BuNC}$ & ${ }^{t} \mathrm{BuNC}$ & $+0.50^{b}$ & - & - \\
\hline${ }^{t} \mathrm{BuNC}$ & ${ }^{t} \mathrm{BuNC}$ & $+0.42^{c}$ & - & - \\
\hline 1-Ad-NC & 1-Ad-NC & +0.57 & +0.59 & +0.65 \\
\hline PhNC & PhNC & +0.62 & +0.67 & +0.73 \\
\hline 2,6--Me $2 \mathrm{py}$ & $\mu_{2}-\mathrm{S}-\mathrm{C}_{2} \mathrm{Ph}_{2} \mathrm{~S}$ & $+0.05,+0.67^{d}$ & - & - \\
\hline MeNC & $1,4-\left(\mathrm{Ph}_{2} \mathrm{P}\right)_{2} \mathrm{C}_{6} \mathrm{H}_{4}$ & $+0.51,+1.26^{e}$ & $+0.52,+0.93^{d}$ & $+0.57,+1.26^{d}$ \\
\hline $\mathrm{CN}^{1-}$ & $\mathrm{CN}^{1-}$ & -0.07 & - & - \\
\hline MeNC & $\mathrm{CN}^{1-}$ & - & +0.27 & +0.26 \\
\hline MeNC & IPr & $+0.41,+1.78^{e}$ & +0.33 & - \\
\hline MeNC & $\mathrm{C}(\mathrm{NMe}) \mathrm{IPr}$ & - & - & +0.28 \\
\hline MeNC & $\mathrm{C}(\mathrm{NHMe})\left(\mathrm{NMe}_{2}\right)$ & +0.35 & - & - \\
\hline
\end{tabular}

${ }^{a}$ Under these conditions, the $\mathrm{Cp}_{2} \mathrm{Fe}^{+} / \mathrm{Cp}_{2} \mathrm{Fe}$ couple consistently occurred at $+540 \mathrm{mV} .{ }^{b} \mathrm{The}$ dithiolene ligand is $\left[\left(\mathrm{CH}_{3} \mathrm{O}-p-\mathrm{C}_{6} \mathrm{H}_{4}\right)_{2} \mathrm{~S}_{2} \mathrm{C}_{2}\right]^{2-}$ rather than $\left[\mathrm{Ph}_{2} \mathrm{C}_{2} \mathrm{~S}_{2}\right]^{2-}$. ${ }^{c}$ The dithiolene ligand is $\left[\mathrm{Me}_{2} \mathrm{~S}_{2} \mathrm{C}_{2}\right]^{2-}$ rather than $\left[\mathrm{Ph}_{2} \mathrm{C}_{2} \mathrm{~S}_{2}\right]^{2-}$. ${ }^{d}$ Quasireversible wave. ${ }^{e}$ Irreversible wave.

for $\left[\left(\mathrm{Ph}_{2} \mathrm{C}_{2} \mathrm{~S}_{2}\right) \mathrm{Ni}(\mathrm{CNMe})\right]_{2}(\mu-1,4-\mathrm{dppb})$, $\left[\left(\mathrm{Ph}_{2} \mathrm{C}_{2} \mathrm{~S}_{2}\right) \mathrm{Ni}(\mathrm{CNMe})\left(\mathrm{C}(\mathrm{NHMe})\left(\mathrm{NMe}_{2}\right)\right.\right.$ trend as $+0.51 \mathrm{~V}>+0.41 \mathrm{~V}>+0.35$ respectively. The basicity of the noncyclic carbene in $\mathbf{2 1}$ is likely greater than that of IPr in $\mathbf{1 8}$ because of a delocalizing effect of the imidazole ring in the latter. The electrochemistry of dimetallic compounds 11-13 is essentially similar to that of monometallic congeners 9 and 10 because their redox-active $M\left(S_{2} C_{2} P_{2}\right)$ end groups are far enough apart as to be effectively independent. Thus, the waves they reveal at $+0.51-0.57 \mathrm{~V}$ are attributed to concurrent one-electron oxidations of the metallodithiolene end-groups such that, as has been found for dimetallic compounds of the composition $\left[\left(\mathrm{R}_{2} \mathrm{C}_{2} \mathrm{~S}_{2}\right) \mathrm{M}\left(\mu_{2}-\mathrm{tpbz}\right) \mathrm{M}\left(\mathrm{S}_{2} \mathrm{C}_{2} \mathrm{R}_{2}\right)\right]^{40-41}$ (tpbz $=1,2,4,5-$ tetrakis(diphenylphosphino)benzene), two remotely coupled radical monoanions are generated.

Compound 8 differs in kind from those of the type $\left[\left(\mathrm{Ph}_{2} \mathrm{C}_{2} \mathrm{~S}_{2}\right) \mathrm{M}(\mathrm{CNMe}) \mathrm{L}\right]$ in having no MeNC ligand. More importantly, while the compound is dimetallic, the bridging interactions are provided by a thiolate sulfur atom from each of the two dithiolene ligands. Consequently, the $\mathrm{Ni}_{2}\left(\mu_{2}-\eta^{1}, \eta^{1}-\mathrm{S}^{\prime}, \eta^{1}-\mathrm{S}^{\prime \prime}-\mathrm{C}_{2} \mathrm{Ph}_{2}\right)_{2}$ core can only be regarded as a single redox-active entity rather than as two independent fragments as in 11-13. The cyclic voltammogram of $\mathbf{8}$ shows a reversible wave at $+0.05 \mathrm{~V}$, substantially less positive by $\sim+0.50$ $\mathrm{V}$ than the oxidations found for compounds 4 . This feature is followed by a quasireversible oxidation at +0.67 (Figure 4). As suggested by symmetry considerations and by its relatively compact core, the HOMO for 8 is largely composed of the $C_{2} S_{2} \pi$ systems of the two identical dithiolene ligands with modest contribution from $\mathrm{Ni}^{2+}$. Delocalization of charge in $[\mathbf{8}]^{1+}$ throughout the $\mathrm{Ni}_{2}\left(\mu_{2}-\eta^{1}, \eta^{1}-\mathrm{S}^{\prime}, \eta^{1}-\mathrm{S}^{\prime \prime}-\mathrm{C}_{2} \mathrm{Ph}_{2}\right)_{2}$ center enables both the milder oxidation potential for $\mathbf{8}$ and, most likely, its capacity to sustain another oxidation 


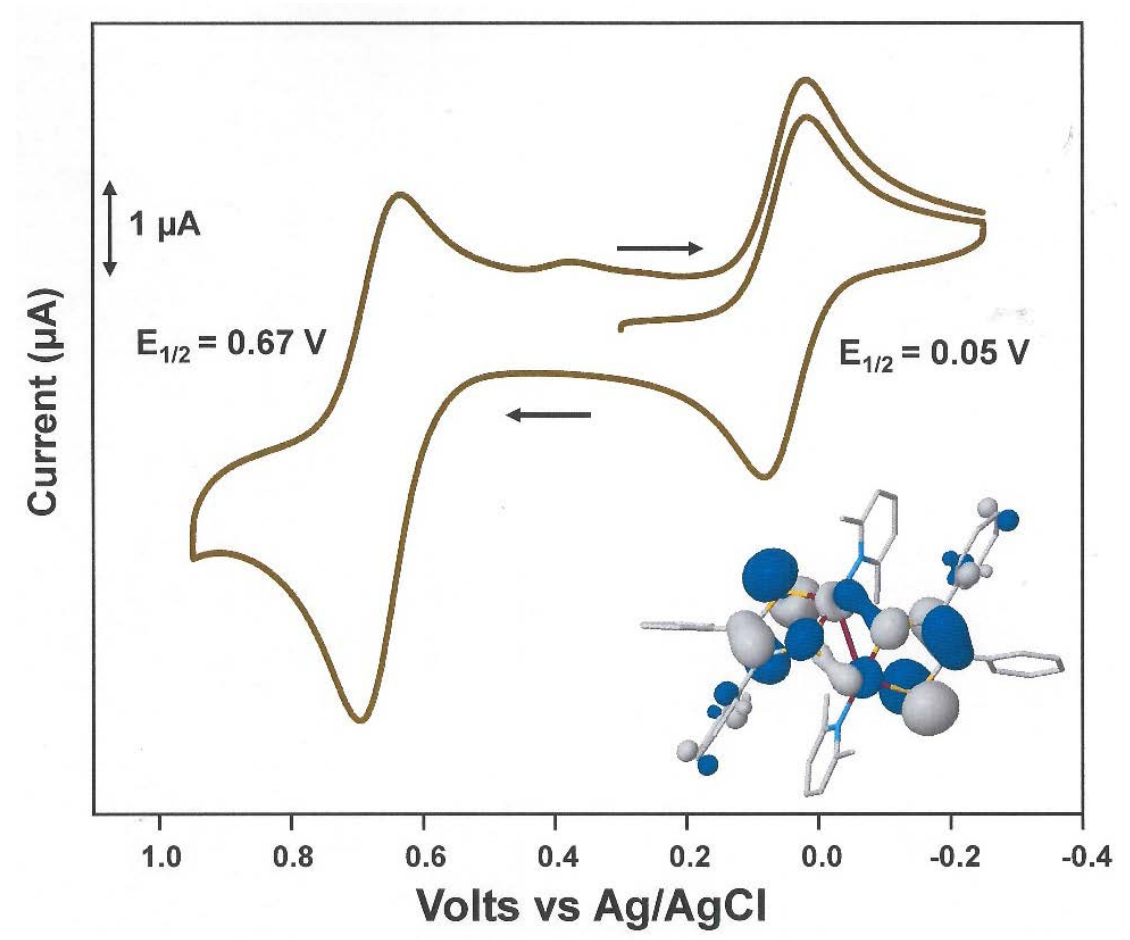

Figure 4. Cyclic voltammogram of $\left[\left(2,6-\mathrm{Me}_{2} \mathrm{py}\right) \mathrm{Ni}\left(\mu_{2}-\eta^{1}, \eta^{1}-\mathrm{S}^{\prime}, \eta^{1}-\mathrm{S}^{\prime \prime}-\right.\right.$ $\left.\left.\mathrm{C}_{2} \mathrm{Ph}_{2}\right)\right]_{2}$ in $\mathrm{CH}_{2} \mathrm{Cl}_{2}$ with $\left[\mathrm{Bu}_{4} \mathrm{~N}\right]\left[\mathrm{PF}_{6}\right]$ supporting electrolyte. The figure inset illustrates the HOMO (0.03 contour level), which is largely comprised of the $\mathrm{C}_{2} \mathrm{~S}_{2}$ portions of the dithiolene ligands.

where compounds $\mathbf{4}$ cannot. Were it to be isolated, $[\mathbf{8}]^{1+}$ is anticipated to show $\mathrm{C}-\mathrm{C}_{\text {chelate }}$ and $\mathrm{S}-\mathrm{C}$ bond lengths between those of the fully reduced ene-1,2-dithiolate and the radical monoanion ((a) and (b), respectively, Scheme 1) since the presumed one-electron oxidation is averaged over two ligands. The LUMO for 8 is calculated as being $\sim 2.8 \mathrm{eV}$ higher in energy than the HOMO and, similar to compounds 46, is $M\left(d_{x^{2}-y^{2}}\right)-L \sigma^{*}$ in nature.

Summary. The principal findings of this work are as follows:

(1) The synthesis of $\left[\left(\mathrm{Ph}_{2} \mathrm{C}_{2} \mathrm{~S}_{2}\right) \mathrm{M}(\mathrm{C} \equiv \mathrm{NR})_{2}\right]\left(\mathrm{M}=\mathrm{Ni}^{2+}, \mathrm{Pd}^{2+}\right.$, or $\mathrm{Pt}^{2+} ; \mathrm{R}=\mathrm{Me}, \mathrm{Bn}, \mathrm{Cy},{ }^{t} \mathrm{Bu}, 1$-adamantyl, $\mathrm{Ph})$, proceed by dithiolene substitution from homoleptic $\left[\left(\mathrm{Ph}_{2} \mathrm{C}_{2} \mathrm{~S}_{2}\right)_{2} \mathrm{M}\right]$. All compounds are air-stable and amenable to purification by column chromatography. Yields of isolated material range from $72-91 \%$ for $\mathrm{M}$ $=\mathrm{Ni}^{2+}, 55-80 \%$ for $\mathrm{M}=\mathrm{Pd}^{2+}$, and 37-52\% for $\mathrm{M}=\mathrm{Pt}^{2+}$, implying stronger metal-dithiolene binding in moving from the first to the third row metal.

(2) Between this report and a preceding communication, all 18 members of the set have been characterized by X-ray crystallography. Progressively greater square planarity is observed in moving from $\mathrm{Ni}^{2+}$ to $\mathrm{Pd}^{2+}$ to $\mathrm{Pt}^{2+}$. 
(3) The $v_{\mathrm{CN}}$ stretching frequencies in $\left[\left(\mathrm{Ph}_{2} \mathrm{C}_{2} \mathrm{~S}_{2}\right) \mathrm{M}(\mathrm{C} \equiv \mathrm{NR})_{2}\right]$ all occur at higher energy than the free ligand and increase in energy as $\mathrm{M}$ varies from $\mathrm{Ni}^{2+}$ to $\mathrm{Pd}^{2+}$ to $\mathrm{Pt}^{2+}$. This trend is rationalized as arising from increasing cationic character for the heavier metals, which tends to promote the linear, charge-separated resonance form of the isonitrile ligand $\left(\overline{: C} \equiv N^{+}-R\right)$ over its bent counterpart $(: C=\ddot{N}-R)$.

(4) Compound types $\left[\left(\mathrm{Ph}_{2} \mathrm{C}_{2} \mathrm{~S}_{2}\right) \mathrm{M}(\mathrm{C} \equiv \mathrm{NR})_{2}\right]$ and $\left[\left(\mathrm{Ph}_{2} \mathrm{C}_{2} \mathrm{~S}_{2}\right) \mathrm{M}(\mathrm{C} \equiv \mathrm{NR}) \mathrm{L}\right]$ sustain a reversible or quasireversible 1-electron oxidation due to the redox-active dithiolene ligand: $\mathrm{Ph}_{2} \mathrm{C}_{2} \mathrm{~S}_{2}{ }^{2-}-\mathrm{e}^{-} \rightarrow \mathrm{Ph}_{2} \mathrm{C}_{2} \mathrm{~S}^{-} \mathrm{S}^{*}$. In the case of $\left[\left(\mathrm{Ph}_{2} \mathrm{C}_{2} \mathrm{~S}_{2}\right) \mathrm{Pt}\left(\mathrm{C} \equiv \mathrm{N}^{t} \mathrm{Bu}\right)_{2}\right]$, treatment with $\left[\left(\mathrm{Br}-p-\mathrm{C}_{6} \mathrm{H}_{4}\right)_{3} \mathrm{~N}\right]\left[\mathrm{SbCl}_{6}\right]$ and crystallization of the isolated product yields centrosymmetric dimeric $\left[\left[\left(\mathrm{Ph}_{2} \mathrm{C}_{2} \mathrm{~S}_{2}\right) \mathrm{Pt}\left(\mathrm{C} \equiv \mathrm{N}^{t} \mathrm{Bu}\right)_{2}\right]_{2}\right]^{2+}$ formed via $\mathrm{Pt} \cdots \mathrm{S}$ axial interactions in a rhombic $(\mathrm{Pt} \cdots \mathrm{S})_{2}$ core.

(5) The $\left[\left(\mathrm{Ph}_{2} \mathrm{C}_{2} \mathrm{~S}_{2}\right) \mathrm{M}(\mathrm{C} \equiv \mathrm{NMe})_{2}\right]$ compounds provide access to new heteroleptic dithiolene compounds by displacement of $\mathrm{MeN} \equiv \mathrm{C}$ with various other ligands $\left(\mathrm{CN}^{-}\right.$, phosphine, carbene). The spectroscopic and electrochemical properties of $\left[\left(\mathrm{Ph}_{2} \mathrm{C}_{2} \mathrm{~S}_{2}\right) \mathrm{M}(\mathrm{C} \equiv \mathrm{NMe}) \mathrm{L}\right]$ trend according to the $\sigma$-donor strength of $\mathrm{L}$.

(6) Differences as function of $\mathrm{M}$ in the pattern of $\mathrm{MeN} \equiv \mathrm{C}$ displacement from $\left[\left(\mathrm{Ph}_{2} \mathrm{C}_{2} \mathrm{~S}_{2}\right) \mathrm{M}(\mathrm{C} \equiv \mathrm{NMe})_{2}\right]$ arise from strengthened $\mathrm{M}-\mathrm{C} \equiv \mathrm{NMe}$ binding in moving from $\mathrm{M}=\mathrm{Ni}^{2+}$ to $\mathrm{Pd}^{2+}$ to $\mathrm{Pt}^{2+}$. Thus, for $\mathrm{M}^{2} \mathrm{Ni}^{2+}$ and $\mathrm{Pd}^{2+}$, the Arduengo carbene $\mathrm{IPr}$ reacts with $\left[\left(\mathrm{Ph}_{2} \mathrm{C}_{2} \mathrm{~S}_{2}\right) \mathrm{M}(\mathrm{C} \equiv \mathrm{NMe})_{2}\right]$ to form $\left[\left(\mathrm{Ph}_{2} \mathrm{C}_{2} \mathrm{~S}_{2}\right) \mathrm{M}(\mathrm{IPr})(\mathrm{C} \equiv \mathrm{NMe})\right]$, while for $\left[\left(\mathrm{Ph}_{2} \mathrm{C}_{2} \mathrm{~S}_{2}\right) \mathrm{Pt}(\mathrm{C} \equiv \mathrm{NMe})_{2}\right]$, IPr attacks the isonitrile carbon to form an $\eta^{1}, \kappa C$-ketenimine ligand in a bent, charge-separated resonance form.

In continuing work, we probe the utility of $\left[\left(\mathrm{Ph}_{2} \mathrm{C}_{2} \mathrm{~S}_{2}\right) \mathrm{M}(\mathrm{C} \equiv \mathrm{NMe})_{2}\right]$ as synthons toward new metallodithiolene compounds, including multi-metallic compounds, that are not accessible by other routes. 


\section{Associated Content}

Supporting Information. Procedures for crystal growth, X-ray diffraction data collection, and structure solution and refinement; tables summarizing unit cell and refinement data (Tables S1-S6; thermal ellipsoid plots with complete atom labelling (Figures S1- S48); spectroscopic, electrochemical, and analytical data for compounds reported (Figures S49-S196); description of computation procedures; coordinates for geometry optimized structures.

Accession Codes. CCDC 1920776-1920790 and CCDC 1995894-1995907 contain the supplementary crystallographic data for this paper. These data can be obtained free of charge via www.ccdc.cam.ac.uk/data_request/cif, or by emailing data_request@ccdc.cam.ac.uk, or by contacting The Cambridge Crystallographic Data Centre, 12 Union Road, Cambridge CB2 1EZ, UK; fax: +44 1223 336033.

\section{Author Information}

Corresponding Authors

*E-mail: donahue@tulane.edu

Notes

The authors declare no competing financial interest.

\section{Acknowledgments.}

The Louisiana Board of Regents (LEQSF-(2002-03)-ENH-TR-67) and the National Science Foundation (MRI: 1228232 and 0619770) are thanked for funding of Tulane University's X-ray crystallography and mass spectrometry instrumentation, and Tulane University is acknowledged for its ongoing assistance with operational costs for the X-ray diffraction facility. The authors (A. O., J. P. D.) gratefully acknowledge support for this project from the National Science Foundation (CHE: 1836589). Ms. Titir Das Gupta is thanked for assistance with addressing a few experimental measurements. 


\section{References}

(1) Schrauzer, G. N.; Mayveg, V. P.; Heinrich, W. Coordination Compounds with Delocalized Ground States. $\alpha$-Dithiodiketone-Substituted Group VI Metal Carbonyls and Related Compounds. J. Am. Chem. Soc. 1966, 88, 5174-5179.

(2) Enemark, J. H.; Cooney, J. J. A.; Wang, J.-J.; Holm, R. H. Synthetic Analogues and Reaction Systems Relevant to the Molybdenum and Tungsten Oxotransferases. Chem. Rev. 2004, 104, 1175-1200.

(3) Nomura, M.; Okuyama, R.; Fujita-Takayama, C.; Kajitani, M. New Synthetic Methods for $\eta^{5}$ Cyclopentadienyl Nickel(III) Dithiolene Complexes Derived from Nickelocene. Organometallics 2005, 24, 5110-5115.

(4) Adams, H.; Morris, M. J.; Morris, S. A.; Motley, J. C. Dithiolene Transfer from Nickel to a Dimolybdenum Centre: the First Dithiolene Alkyne Complex. J. Organomet. Chem. 2004, 689, 522-527.

(5) Adams, H.; Gardner, H. C.; McRoy, R. A.; Morris, M. J.; Motley, J. C.; Torker, S. Heterometallic Dithiolene Complexes Formed by Stepwise Displacement of Cyclopentadienyl Ligands from Nickelocene with $\mathrm{CpMo}\left(\mathrm{S}_{2} \mathrm{C}_{2} \mathrm{Ph}_{2}\right)_{2}$. Inorg. Chem. 2006, 45, 10967-10975.

(6) Adams, H.; Coffey, A. M.; Morris, M. J.; Morris, S. A. Efficient Transfer of Either One or Two Dithiolene Ligands from Nickel to Ruthenium: Synthesis and Crystal Structures of $\left[\mathrm{Ru}(\mathrm{SCR}=\mathrm{CPhS})_{2}\left(\mathrm{PPh}_{3}\right)\right]$ and $\left[\mathrm{RuCl}_{2}(\mathrm{SCR}=\mathrm{CPhS})\left(\mathrm{PPh}_{3}\right)_{2}\right](\mathrm{R}=\mathrm{Ph}, \mathrm{H})$. Inorg. Chem. 2009, 48, 1194511953.

(7) Adams, H.; Grimes, L.; Morris, M. J.; Robertson, C. C. Dithiolene Transfer to the Molybdenum Nitrosyl Complex [CpMo(CO) $\left.)_{2}(\mathrm{NO})\right]$ : Formation of Bimetallic Complexes. J. Organomet. Chem. 2018, 87, 73-79.

(8) Adams, H.; Morris, M. J.; Robertson, C. C.; Tunnicliffe, H. C. I. Synthesis of Mono- and Diiron Dithiolene Complexes as Hydrogenase Models by Dithiolene Transfer Reactions, Including the Crystal Structure of $\left[\left\{\mathrm{Ni}\left(\mathrm{S}_{2} \mathrm{C}_{2} \mathrm{Ph}_{2}\right)\right\}_{6}\right]$. Organometallics 2019, 38, 665-676.

(9) Mayweg, V. P.; Schrauzer, G. N. Bis-adducts of Group VIII Metal Bisdithiobenzil Complexes with Phosphines. Chem. Commun. 1966, 640-641.

(10) Obanda, A.; Martinez, K.; Schmehl, R. H.; Mague, J. T.; Rubtsov, I. V.; Macmillan, S. N.; Lancaster, K. M.; Sproules, S.; Donahue, J. P. Expanding the Scope of Ligand Substitution from $\left[\mathrm{M}\left(\mathrm{S}_{2} \mathrm{C}_{2} \mathrm{Ph}_{2}\right)_{2}\right](\mathrm{M}=$ $\mathrm{Ni}^{2+}, \mathrm{Pd}^{2+}, \mathrm{Pt}^{2+}$ ) to Afford New Heteroleptic Dithiolene Complexes. Inorg. Chem. 2017, 56, 10257-10267.

(11) Hanson, G. R.; Gates, K. E.; Noble, C. J.; Griffin, M.; Mitchell, A.; Benson, S. XSophe-SopheXeprView ${ }^{\circledR}$. A Computer Simulation Software Suite (v. 1.1.3) for the Analysis of Continuous Wave EPR Spectra. J. Inorg. Biochem. 2004, 98, 903-916.

(12) Schuster, R. E.; Scott, J. E.; Casanova, J., Jr. Methyl Isocyanide. Org. Synth. 1966, 46, 75-77.

(13) Eckert, H.; Nestl, A.; Ugi, I. Phenylisonitrile. In Encyclopedia of Reagents for Organic Synthesis; Wiley \& Sons: 2001.

(14) Gokel, G. W.; Widera, R. P.; Weber, W. P. Phase-Transfer Hofmann Carbylamine Reaction: tert-Butyl Isocyanide. Org. Synth. 1976, 55, 96-99.

(15) Schmidtendorf, M.; Pape, T.; Hahn, F. E. Molecular Rectangles from Platinum(II) and Bridging Dicarbene, Diisocyanide, and 4,4'-Bipyridine Ligands. Dalton Trans. 2013, 42, 16128-16141.

(16) Bantreil, X.; Nolan, S. P. Synthesis of $N$-Heterocyclic Carbene Ligands and Derived Ruthenium Olefin Metathesis Catalytst. Nat. Protoc. 2011, 6, 69-77.

(17) Baldwin, R. A.; Cheng, M. T. Arylenebis(Tertiary Phosphines) and -(Phosphinic Acids. J. Org. Chem. 1967, 32, 1572-1577.

(18) Amarego, W. L. F.; Perrin, D. D. Purification of Laboratory Chemicals, 4th ed; ButterworthHeinemann: Oxford, U.K., 2000.

(19) Connelly, N. G.; Geiger, W. E. Chemical Redox Agents for Organometallic Chemistry. Chem. Rev. 1996, 96, 877-910. 
(20) Arumugam, K.; Shaw, M. C.; Chandrasekaran, P.; Villagrán, D.; Gray, T. G.; Mague, J. T.; Donahue, J. P. Synthesis, Structures and Properties of 1,2,4,5-Benzenetetrathiolate Linked Group 10 Metal Complexes. Inorg. Chem. 2009, 48, 10591-10607.

(21) Robertson, S. D.; Slawin, A. M. Z.; Woollins, J. D. Constructing Multimetallic Systems with the Naphthalene-1,8-bis(thiolato) Ligand. Eur. J. Inorg. Chem. 2007, 247-253.

(22) Shin, R. Y. C.; Tan, G. K.; Koh, L. L.; Vittal, J. J.; Goh, L. Y.; Webster, R. D. Metallophilicity in Annular $\mathrm{Ru}_{2} \mathrm{M}_{2}$ Derivatives of (HMB)Ru${ }^{\mathrm{II}}$ (tpdt) versus Bis- $\eta 2$-Dithiolate Bonding in $\mathrm{Ru}_{2} \mathrm{M}$ Derivatives of $\mathrm{Cp} * \mathrm{Ru}^{\mathrm{III}}$ (tpdt) $\left(\mathrm{HMB}=\eta^{6}-\mathrm{C}_{6} \mathrm{Me}_{6} ; \mathrm{Cp}^{*}=\eta^{5}-\mathrm{C}_{5} \mathrm{Me}_{5} ; \mathrm{M}=\mathrm{Cu}^{\mathrm{I}}, \mathrm{Ag}^{\mathrm{I}, \mathrm{II}}\right.$, $\mathrm{Au}{ }^{\mathrm{I}}$; tpdt = 3-thiapentane-1,5dithiolate). Organometallics 2005, 24, 539-551.

23) Yu, R.; Arumugam, K.; Manepalli, A.; Tran, Y.; Schmehl, R.; Jacobsen, H.; Donahue, J. P. Reversible, Electrochemically Controlled Binding of Phosphine to Iron and Cobalt Bis(dithiolene) Complexes. Inorg. Chem. 2007, 46, 5131-5133.

(24) Sproules. S.; Wieghardt, K. o-Dithiolene and o-Aminothiolate Chemistry of Iron: Synthesis, Structure and Reactivity. Coord. Chem. Rev. 2010, 254, 1358-1382.

(25) Pap, J. S.; Benedito, F. L.; Bothe, E.; Bill, E.; DeBeer George, S.; Weyhermüller, T.; Wieghardt, K. Dimerization Processes of Square Planar [Pt ${ }^{\mathrm{II}}\left(\mathrm{tbpy}^{\mathrm{b}}\right)\left(\right.$ dithiolato $\left.\left.^{\circ}\right)\right]^{+}$Radicals. Inorg. Chem. 2007, 46, 41874196.

(26) Pop. F.; Branzea, D. G.; Cauchy, T.; Avarvari, N. Bimetallic Neutral Palladium(II) Bis(dithiolene) Complex: Unusual Synthesis, Structural and Theoretical Study. CR Chim. 2012, 15, 904-910.

(27) Chandrasekaran, P.; Greene, A. F.; Lillich, K.; Capone, S.; Mague, J. T.; DeBeer, S.; Donahue, J. P. A Structural and Spectroscopic Investigation of Octahedral Platinum Bis(dithiolene)phosphine Complexes: Platinum Dithiolene Internal Redox Chemistry Induced by Phosphine Association. Inorg. Chem. 2014, 53, 9192-9205.

(28) Hudnall, T. W.; Moorhead, E. J.; Gusev, D. G.; Bielawski, C. W. N,N'-Diamidoketenimines via Coupling of Isocyanides to an $N$-Heterocyclic Carbene. J. Org. Chem. 2010, 75, 2763-2766.

(29) Enders, D.; Niemeier, O.; Henseler, A. Organocatalysis by N-Heterocyclic Carbenes. Chem. Rev. 2007, 107, 5606-5655.

(30) Coyle, J. P.; Sirianni, E. R.; Korobkov, I.; Yap, G. P. A.; Dey, G.; Barry, S. T. Study of Monomeric Copper Complexes Supported by N-Heterocyclic and Acyclic Diamino Carbenes. Organometallics 2017, 36, 2800-2810.

(31) Holthoff, J. M.; Engelage, E.; Kowsari, A. B.; Huber, S. M.; Weiss, R. Noble Metal Corrosion: Halogen Bonded Iodocarbenium Iodides Dissolve Elemental Gold - Direct Access to Gold-Carbene Complexes. Chem. Eur. J. 2019, 25, 7480-7484.

(32) Cotton, F. A.; Zingales, F. The Donor-Acceptor Properties of Isonitriles as Estimated by Infrared Study. J. Am. Chem. Soc. 1961, 83, 351-355.

(33) Barybin, M. V.; Young, V. G., Jr.; Ellis, J. E. First Paramagnetic Zerovalent Transition Metal Isocyanides. Synthesis, Structural Characterizations, and Magnetic Properties of Novel Low-Valent Isocyanide Complexes of Vanadium. J. Am. Chem. Soc. 2000, 122, 4678-4691.

(34) Lockwood, M. A.; Fanwick, P. E.; Rothwell, I. P. Reactivity of a Tungsten(II) Aryloxide with Isocyanides and Isocyanates. Organometallics 1997, 16, 3574-3575.

(35) Kucera, B. E.; Roberts, C. J.; Young, V. G., Jr.; Brennessel, W. W.; Ellis, J. E. Niobium Isocyanide Complexes, $\mathrm{Nb}(\mathrm{CNAr})_{6}$, with $\mathrm{Ar}=2$,6-Dimethylphenyl (Xyl), a Diamagnetic Dimer Containing Four Reductively Coupled Isocyanides, and $\mathrm{Ar}=2$,6-Diisopropylphenyl (Diip), a Paramagetic Monomer Analogous to the Highly Unstable Hexacarbonylniobium(0). Acta Crystallogr., Sect C. 2019, 75, 12591265. 
(36) Chakarawet, K.; Davis-Gilbert, Z. W.; Harstad, S. R.; Young, V. G., Jr.; Long, J. R.; Ellis, J. E. Ta(CNDipp) 6 : An Isocyanide Analogue of Hexacarbonyltantalum(0). Angew. Chem. Int. Ed. 2017, 56, 10577-10581.

(37) Sattler, W.; Ener, M. E.; Blakemore, J. D.; Rachford, A. A.; LaBeaume, P. J.; Thackeray, J. W.; Cameron, J. F.; Winkler, J. R.; Gray, H. B. Generation of Powerful Tungsten Reductants by Visible Light Excitation. J. Am. Chem. Soc. 2013, 135, 10614-10617.

(38) Brink, A.; Roodt, A.; Steyl, G.; Visser, H. G. Steric vs. electronic anomaly observed from iodomethane oxidative addition to tertiary phosphine modified rhodium(I) acetylacetonato complexes following progressive phenyl replacement by cyclohexyl $\left[\mathrm{PR}_{3}=\mathrm{PPh}_{3}, \mathrm{PPh}_{2} \mathrm{Cy}, \mathrm{PPhCy}_{2}, \mathrm{PCy}_{3}\right]$. Dalton Trans. 2010, 39, 5572-5578.

(39) Chen, L.; Ren, P.; Carrow, B. P. Tri(1-adamantyl)phosphine: Expanding the Boundary of ElectronReleasing Character Available to Organophosphorus Compounds. J. Am. Chem. Soc. 2016, 138, 63926395.

(40) Arumugam, K.; Shaw, M. C.; Mague, J. T.; Bill, E.; Sproules, S.; Donahue, J. P. Long-Range Spin Coupling: A Tetraphosphine-Bridged Palladium Dimer. Inorg. Chem. 2011, 50, 2995-3002.

(41) Arumugam, K.; Selvachandran, M.; Obanda, A.; Shaw, M. C.; Chandrasekaran, P.; Caston Good, S. L.; Mague, J. T.; Sproules, S.; Donahue, J. P. Redox-Active Metallodithiolene Groups Separated by Insulating Tetraphosphinobenzene Spacers. Inorg. Chem. 2018, 57, 4023-4038. 
For Table of Contents Use Only

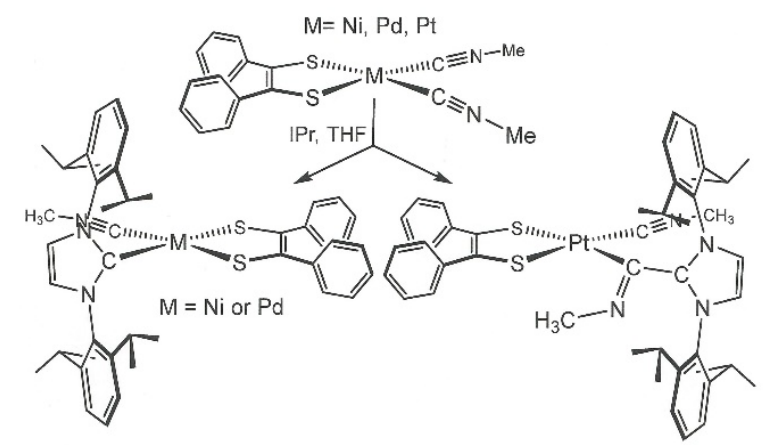

